\title{
Multiobjective Differential Evolution Enhanced with Principle Component Analysis for Constrained Optimization ${ }^{\text {th }}$
}

\author{
Wei Huang ${ }^{\mathrm{a}}$, Tao $\mathrm{Xu}^{\mathrm{b}}$, Kangshun $\mathrm{Li}^{\mathrm{c}}$, Jun $\mathrm{He}^{\mathrm{d}, *}$ \\ ${ }^{a}$ Tianjin Key Laboratory of Intelligent and Novel Software Technology, and School of Computer Science and Engineering, \\ Tianjin University of Technology, China \\ ${ }^{b}$ Department of Computer Science, Aberystwyth University, Aberystwyth SY23 3DB, UK \\ ${ }^{c}$ College of Mathematics and Informatics, South China Agricultural University, Guangzhou 510642, China \\ ${ }^{d}$ School of Science and Technology, Nottingham Trent University, Nottingham NG11 8NS, UK
}

\begin{abstract}
Multiobjective evolutionary algorithms (MOEAs) have been successfully applied to a number of constrained optimization problems. Many of them adopt mutation and crossover operators from differential evolution. However, these operators do not explicitly utilise features of fitness landscapes. To improve the performance of algorithms, this paper aims at designing a search operator adapting to fitness landscapes. Through an observation, we find that principle component analysis (PCA) can be used to characterise fitness landscapes. Based on this finding, a new search operator, called PCA-projection, is proposed. In order to verify the effectiveness of PCA-projection, we design two algorithms enhanced with PCA-projection for solving constrained optimization problems, called PMODE and HECO-PDE, respectively. Experiments have been conducted on the IEEE CEC 2017 competition benchmark suite in constrained optimisation. PMODE and HECO-PDE are compared with the algorithms from the IEEE CEC 2018 competition and another recent MOEA for constrained optimisation. Experimental results show that an algorithm enhanced with PCA-projection performs better than its corresponding opponent without this operator. Furthermore, HECO-PDE is ranked first on all dimensions according to the competition rules. This study reveals that decomposition-based MOEAs, such as HECO-PDE, are competitive with best single-objective and multiobjective evolutionary algorithms for constrained optimisation, but MOEAs based on non-dominance, such as PMODE, may not.
\end{abstract}

Keywords: constrained optimization; multiobjective optimization; principle component analysis; differential evolution; fitness landscape.

\section{Introduction}

Optimization problems in the real world often contain different types of constraints. In mathematics, a constrained optimization problem (COP) can be formulated as

$$
\begin{aligned}
& \min f(\mathbf{x}), \quad \mathbf{x}=\left(x_{1}, \cdots, x_{n}\right) \in \mathbb{R}^{n}, \\
& \text { subject to }\left\{\begin{array}{l}
\mathbf{x} \in \Omega=\left\{\mathbf{x} \mid L_{i} \leq x_{i} \leq U_{i}, i=1, \cdots, n\right\}, \\
g_{i}(\mathbf{x}) \leq 0, \quad i=1, \cdots, q, \\
h_{j}(\mathbf{x})=0, \quad j=1, \cdots, r,
\end{array}\right.
\end{aligned}
$$

\footnotetext{
This work was supported in part by the National Natural Science Foundation of China under Grant 61673295 and Grant 61573157, and in part by the Natural Science Foundation of Tianjin under Grant 18JCYBJC85200.

* Corresponding author

Email addresses: huangwabc@163.com (Wei Huang), tax2@aber.ac.uk (Tao Xu), likangshun@sina.com (Kangshun Li), jun.he@ntu.ac.uk (Jun He)
} 
where $\mathbf{x} \in \Omega$ is the bounded constraint. $L_{i}$ and $R_{i}$ denote lower and upper boundaries respectively. $g_{i}(\mathbf{x}) \leq 0$ is the $i$ th inequality constraint. $h_{j}(\mathbf{x})=0$ is the $j$ th equality constraint.

There exist a variety of evolutionary algorithms (EAs) for solving COPs, which employ different constraint handling techniques, such as the penalty function method, feasibility rule, repair method and multiobjective optimization $[1,2,3]$. This paper focuses on the multi-objective optimization method [4], which is to convert a COP into a multi-objective optimization problem without any constraint. The advantage of using this method is no need to handle constraints in a special way, because constraints themselves are converted into objectives and a COP will be solved by MOEAs.

The converted problem often is a bi-objective optimization problem $[5,6]$ :

$$
\min \mathbf{f}=(f(\mathbf{x}), v(\mathbf{x})),
$$

in which one objective is the original objective function $f(\mathbf{x})$ and the other is the degree of violating the constraints $v(\mathbf{x})$.

$$
v(\mathbf{x})=\sum_{i} v_{i}^{g}(\mathbf{x})+\sum_{j} v_{j}^{h}(\mathbf{x}) .
$$

The first part in the formula is the sum of the degree of violating an inequality constraint, given by

$$
v_{i}^{g}(\mathbf{x})=\max \left\{0, g_{i}(\mathbf{x})\right\}, \quad i=1, \cdots, q .
$$

The second part is the sum of the degree of violating an equal constraint, given by

$$
v_{j}^{h}(\mathbf{x})=\max \left\{0,\left|h_{j}(\mathbf{x})\right|-\delta\right\}, \quad j=1, \cdots, r,
$$

where $\delta$ is a tolerance allowed for the equality constraint.

Many MOEAs have been proposed for solving constrained optimisation problems [4]. Most of them adopt mutation and crossover operators from differential evolution (DE). However, these operators do not explicitly utilise characteristics of fitness landscapes. Intuitively, a search operator which adapts to fitness landscapes may be more efficient than those without adaptation. A recent theoretical study also claims that in terms of the time-based fitness landscape, unimodal functions are the easiest and deceptive functions to EAs are the hardest [7]. Therefore, it is important to design a landscape-adaptive search operator which may improve the performance of EAs.

Our idea is to enhance EAs with principle component analysis (PCA). There exist a suite of studies which have shown PCA may improve the performance of EAs. Munteanu and Lazarescu [8] designed a PCA-mutation operator and claimed that PCA-mutation is more successful in maintaining population diversity during search. Because of PCA's inherent capability of rebuilding a new coordinate system, Li et al. [9] applied PCA to the design of crossover for reducing correlations among variables. PCA was used in particle swam optimization (PSO) to mine population information for promising principal component directions $[10,11,12]$. This information is utilized in velocity vectors of particles. Because PCA is a powerful tool in dimensional reduction, it helped EAs solve high dimensional optimization problems [13, 14]. Besides its application in designing search operators, local principal component analysis is used for building a regularity model in multiobjective estimation of distribution algorithms $[15,16]$. However, the number of references of applying PCA to EAs is still very small and it is worth making further investigations.

In this paper, we design a new search operator adapting to fitness landscapes with the aid of PCA. PCA is used to identify the maximal variance direction in a population. Given a "valley" fitness landscape in the 3-dimensional space, we observe that the direction obtained by PCA is consistent with the valley direction. Based on this observation, we design a new search operator, called PCA-projection. The research question of this paper is whether a MOEA enhanced with PCA-projection is able to outperform its rival without this operator or other state-of-arts EAs. To answer this question, we design two MOEAs enhanced with PCA-projection, conduct experiments on the IEEE CEC 2017 benchmark suit for constrained optimisation competition [17] and compare them with EAs from the CEC 2018 competition [18]. 
The remainder of this paper is organised as follows. Section 2 reviews MOEAs for constrained optimisation. Section 3 introduces related work in differential evolution and PCA's applications in EAs. Section 4 explains PCA-projection in detail. Section 5 designs two EAs enhanced with PCA-projection. Section 6 reports comparative experimental results. Section 7 concludes this paper.

\section{Literature Review of Multiobjective Evolutionary Algorithms for Constrained Optimisation}

The idea of applying MOEAs to constrained optimisation has attracted researchers' interest in last two decades. Surry and Radcliff [5] proposed constrained optimization by multi-objective genetic algorithms. They considered a COP in a dual perspective, as a constraint satisfaction problem and an unconstrained optimization problem. Coello [19] introduced the concept of non-dominance to handle constraints into the fitness function of a genetic algorithm. Feasible individuals are ranked higher than infeasible ones, while infeasible individuals with a lower degree of constraint violation is ranked higher than those with a higher degree. Zhou et al. [6] converts a COP to a two-objective optimization model composed of the original objective function and the degree function violating the constraints. Then they designed a real-coded genetic algorithm based on Pareto strength and Minimal Generation Gap model.

Most MOEAs for constrained optimisation belong to the category of MOEAs based on non-dominance or Pareto ranking. Venkatraman and Yen [20] proposed a two-phase genetic algorithm framework for solving COPs. In the first phase, a COP is treated as a constraint satisfaction problem. In the second phase, a COP is treated as a bi-objective optimization problem with the simultaneous optimization of the objective function and the satisfaction of the constraints. Then the Non-Dominated Sorting Genetic Algorithm (NSGA-II) is used. Cai and Wang [21, 22] combined multiobjective optimization with differential evolution (CMODE) to solve COPs which is based on the two-objective model. The search is guided by infeasible solution archiving and replacement mechanism. Furthermore, they provided a dynamic hybrid framework [23], which consists of global search and local search models. More recently, Gao and Yen et al. [24] considered COPs as a bi-objective optimization problem, where the first objective is the reward function or actual cost to be optimized, while the second objective is the constraint violations degree. Gao et al. [25] proposed a reverse comparison strategy based on multi-objective dominance concept. That strategy converted the original COPs to a multi-objective problem with one constraint, and weeds out worse solutions with smaller fitness value regardless of its constraints violation. Li et al. [26] and Zeng et al. [27] converted a COP into a dynamic constrained many-objective optimization problem, and considered three types of MOEAs, i.e., Pareto ranking-based, decomposition-based, and hype-volume indicator-based to instantiate the framework.

Recently, MOEAs based on objective decomposition have been applied to constrained optimisation. Xu et al. [28] constructed several helper objective functions using the weighted sum method but with static weights. Then they employed DE for optimising optimisation subproblems. Wang et al. [29] considered the weighted sum approach with dynamical weight to decompose the problem (21) and also applied DE to solve them. Peng et al. [30] adopted the Chebyshev approach in objective decomposition with biased dynamic weights.

The purpose of using MOEAs for constrained optimisation is to seek the optimal feasible solution, but not to generate a uniformly distributed Pareto front. Therefore, we guess that decomposition-based MOEAs are more flexible than MOEAs based on non-dominance or Pareto ranking, because through biased dynamic weights, we may adjust the search direction of decomposition-based MOEAs. At the beginning, a MOEA searches different directions, but at the later stage, it focuses more on the direction towards the optimal feasible solution.

\section{Two Pieces of Related Work}

Our work is linked to classical DE [31] and the application of PCA in EAs [8]. This section reviews them one by one. 


\subsection{Differential Evolution}

$\mathrm{DE}$ is a popular EA for solving continuous optimization problems [31]. In $\mathrm{DE}$, a population $P$ is represented by $\mu n$-dimensional vectors:

$$
\begin{aligned}
P & =\left\{\mathbf{x}_{1}, \cdots, \mathbf{x}_{\mu}\right\}, \\
\mathbf{x}_{i} & =\left(x_{i, 1}, x_{i, 2}, \cdots, x_{i, n}\right)^{T}, i=1,2, \cdots, \mu,
\end{aligned}
$$

where $\mu$ is the population size. Initial individuals are chosen randomly from $\left[L_{i}, U_{i}\right]^{n}$. An initial individual $\mathbf{x}=\left(x_{1}, \cdots, x_{n}\right)^{T}$ is generated at random as follows:

$$
x_{i}=L_{i}+\left(U_{i}-L_{i}\right) \times \text { rand }, \quad i=1, \cdots, n,
$$

where rand is the random number $[0,1]$.

The DE algorithm consists of three operations: mutation, crossover and selection, which are described as follows [31, 28].

Mutation: for each individual $\mathbf{x}_{i}$ where $i=1, \cdots, \mu$, a mutant vector $\mathbf{v}_{i}=\left(v_{i, 1}, v_{i, 2}, \cdots, v_{i, n}\right)$ is generated by

$$
\mathbf{v}_{i}=\mathbf{x}_{r 1}+F \cdot\left(\mathbf{x}_{r 2}-\mathbf{x}_{r 3}\right)
$$

where individuals $\mathbf{x}_{r 1}, \mathbf{x}_{r 2}, \mathbf{x}_{r 3}$ are chosen from $P$ at random but mutually different. They are also chosen to be different from $\mathbf{x}_{i} . F$ is a real and constant factor from $[0,2]$ which controls the amplification of the differential variation $\mathbf{x}_{r 2}-\mathbf{x}_{r 3}$. In case $\mathbf{v}_{i}$ is out of the interval $\left[L_{i}, U_{i}\right]$, the mutation operation is repeated until $\mathbf{v}_{i}$ falls in $\left[L_{i}, U_{i}\right]$.

Crossover: in order to increase population diversity, crossover is also used in DE. The trial vector $\mathbf{u}_{i}$ is generated by mixing the target vector $\mathbf{x}_{i}$ with the mutant vector $\mathbf{v}_{i}$.

Trial vector $\mathbf{u}_{i}=\left(u_{i, 1}, u_{i, 2}, \cdots, u_{i, n}\right)$ is constructed as follows:

$$
u_{i, j}=\left\{\begin{array}{ll}
v_{i, j}, & \text { if } \operatorname{rand}_{j}(1,0) \leq C R \text { or } j=j_{\text {rand }}, \\
x_{i, j}, & \text { otherwise, }
\end{array} \quad j=1, \cdots, n,\right.
$$

where $\operatorname{rand}_{j}(0,1)$ is a uniform random number from $[0,1]$. Index $j_{\text {rand }}$ is randomly chosen from $\{1, \cdots, n\} . C R \in[0,1]$ denotes the crossover constant which has to be determined by the user. In addition, the condition " $j=j_{\text {rand }}$ " is used to ensure the trial vector $\mathbf{u}_{i}$ gets at least one parameter from vector $\mathbf{v}_{i}$.

Selection: a greedy criterion is used to decide whether the offspring generated by mutation and crossover should replace its parent. Trail vector $\mathbf{u}_{i}$ is compared to target vector $\mathbf{x}_{i}$, then the better one will be reserved to the next generation.

Notice that both mutation and crossover operators do not explicitly utilise features of fitness landscapes.

There exist several variants of DE algorithms. A classical DE algorithm is the DE/Rand/1/bin DE [32] which is illustrated below.

1: initialize a population $P=\left\{\mathbf{x}_{1}, \cdots, \mathbf{x}_{\mu}\right\}$;

2: calculate fitness values of each individual in $P$;

3: while the terminal condition is not satisfied do

4: $\quad$ for $i=1, \cdots, \mu$ do

5: $\quad$ randomly select three individuals $\mathbf{x}_{r 1}, \mathbf{x}_{r 2}, \mathbf{x}_{r 3}$ from $P$ at random, such that $r_{1} \neq r_{2} \neq r_{3} \neq i$;

6: $\quad$ implement mutation and crossover and generate a child $\mathbf{u}_{i}$ of $\mathbf{x}_{i}$;

7: $\quad$ calculate fitness value $f\left(\mathbf{u}_{i}\right)$;

8: $\quad$ if $f\left(\mathbf{u}_{i}\right) \leq f\left(\mathbf{x}_{i}\right)$ then

9: $\quad \mathbf{x}_{i} \leftarrow \mathbf{u}_{i}$

10: $\quad$ end if

11: $\quad$ end for

12: end while 


\subsection{Application of Principle Component Analysis in Evolutionary algorithms}

It is an interesting idea to apply PCA to the design of EAs but so far only a few research papers can be found on this topic. Munteanu and Lazarescu's work [8] designed a mutation operator based on PCA. They claimed that a PCA-mutation genetic algorithm (GA) is more successful in maintaining population diversity during search. Their experimental results show that a GA with the PCA-mutation obtained better solutions compared to solutions found using GAs with classical mutation operators for a filter design problem.

Munteanu and Lazarescu [8] designed a new mutation operator on a projection search space generated by PCA, rather than the original space. Their PCA mutation is described as follows. A population with $\mu$ individuals is represented by an $n \times \mu$ matrix $\mathbf{X}=\left[\mathbf{x}_{1}, \cdots, \mathbf{x}_{\mu}\right]$ where $n$ is the space dimension and $\mu$ the population size. Each $\mathbf{x}$ is an individual represented by a column vector.

1: From the data set $\mathbf{X}$, calculate the $n \times n$ covariance matrix $\boldsymbol{\Sigma}$ :

$$
\boldsymbol{\Sigma}=\mathbb{E}\left[(\mathbf{x}-\mathbf{m})(\mathbf{x}-\mathbf{m})^{T}\right]
$$

where $\mathbf{m}=\mathbb{E}[\mathbf{x}]$ which is the mean over $\mathbf{x}_{1}, \cdots, \mathbf{x}_{\mu}$.

2: Given the co-variance matrix $\boldsymbol{\Sigma}$, compute its eigenvectors $\mathbf{v}_{1}, \cdots, \mathbf{v}_{n}$ and sort them in the order of the corresponding eigenvalues of these eigenvectors from high to low. Form a $n \times n$ matrix $\mathbf{V}=\left[\mathbf{v}_{1}, \cdots, \mathbf{v}_{n}\right]$.

3: Calculate the projection of the data set $\mathbf{X}$ using the orthogonal basis $\mathbf{v}_{1}, \cdots, \mathbf{v}_{n}$ and obtain a projected population, represented by the matrix $\mathbf{Y}=\left[\mathbf{y}_{1}, \cdots, \mathbf{y}_{n}\right]^{T}$ :

$$
\mathbf{y}_{i}=\mathbf{V}^{T}\left(\mathbf{x}_{i}-\mathbf{m}\right) .
$$

4: Compute the squared length of the projections along each direction $\mathbf{v}_{i}$, that is,

$$
\left\|L_{i}\left(x_{j}\right)\right\|^{2}=y_{i, j}^{2}, \quad i=1, \cdots, n, \quad j=1, \cdots, \mu .
$$

5: Choose quantities $c_{i, j}$ randomly between 0 and $c_{\max }$ where $c_{\max }$ is a constant parameter of the mutation operator such that $c_{i-1, j} \leq c_{i, j}$ for $i=1, \cdots, n$.

6: The mutation operator adds the quantities $c_{i, j}$ to each projected squared coordinate as follows:

$$
\left\|L_{i}^{\prime}\left(x_{j}\right)\right\|^{2}=\left\|L_{i}\left(x_{j}\right)\right\|^{2}+c_{i, j} .
$$

7: Compute the sign of each element in the matrix $\mathbf{Y}$, which is represented by the matrix $\operatorname{signum}(\mathbf{Y})$.

8: Generate the child $\mathbf{y}_{i}^{\prime}$ from $\mathbf{y}_{i}$ as follows: $y_{i, j}^{\prime}$ equals to the square roots of the mutated square projections $\left\|L_{i}^{\prime}\left(x_{j}\right)\right\|^{2}$ multiplied by the corresponding sign $\operatorname{signum}\left(y_{i, j}\right)$.

9: Obtain the mutated point in the original search space:

$$
\mathbf{x}_{i}^{\prime}=\mathbf{V y}_{i}^{\prime}+\mathbf{m}
$$

Notice that the above PCA-mutation doesn't reduce the data set $\mathbf{X}$ into a lower dimension space, instead $\mathbf{X}$ and $\mathbf{Y}$ have the same dimension. This PCA-mutation aims to conduct mutation in the projection space rather than the original space. However the dimensions of the projection space and original space are the same.

\section{A New Search Operator: PCA-projection}

In order to improve the performance of EAs, we propose a new search operator, called PCA-projection, which is able to adapt to fitness landscapes. 


\subsection{Principle Component Analysis and Valley Direction}

Although PCA-mutation proposed in [8] was efficient for a filter design problem, it has a disadvantage. PCA-mutation still acts on the same dimension space as the original search space. Thus, as the population size increases, the calculation of eigenvalues and eigenvectors in PCA becomes more and more expensive. In this paper, we propose a different PCA-search operator in which PCA is only applied to several selected points. A question is how to select points from a population for implementing PCA? The solution relies on the "valley" concept.

In the 3-dimensional space, a valley is intuitive which means a low area between two hills or mountains. However, this definition is really fuzzy in high-dimensional spaces. What does a valley in a higher dimensional space mean? How to identify the location of a valley? So far there exist no clear mathematical definition about the valley. In this paper, we study the valley landscape using PCA and find that PCA provides a statistic method of identifying the valley direction.

Let's explain our idea using the well-known Rosenbrock function:

$$
f(x, y)=(1-x)^{2}+100\left(y-x^{2}\right), \quad-1<x<2,-1<y<2
$$

Its minimum point is at $(1,1)$ with $f(1,1)=0$. Fig. 1a shows the contour graph of Rosenbrock function. From Fig. 1a, it is obvious that a deep valley exists on this landscape. But how to identify the valley?

In the following we show a statistical method of calculating the valley direction. First we sample 20 points at random and select 6 points with smallest function values from the population. Fig. 1b depicts that these 6 points (labelled by squared points) are closer to the valley than other points.

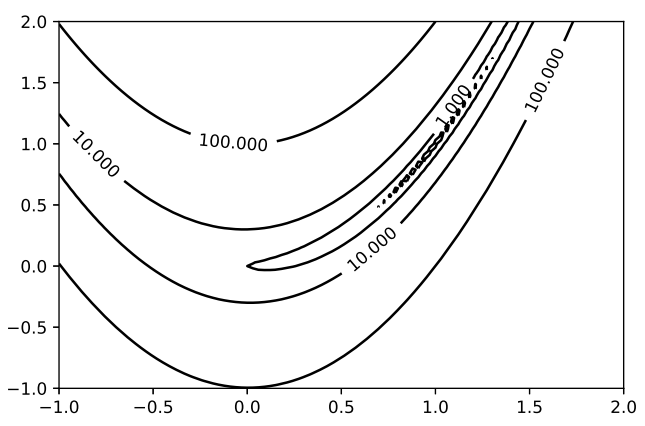

(a)

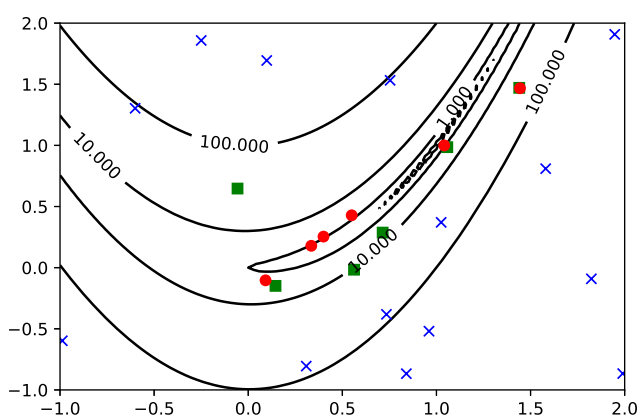

(c)

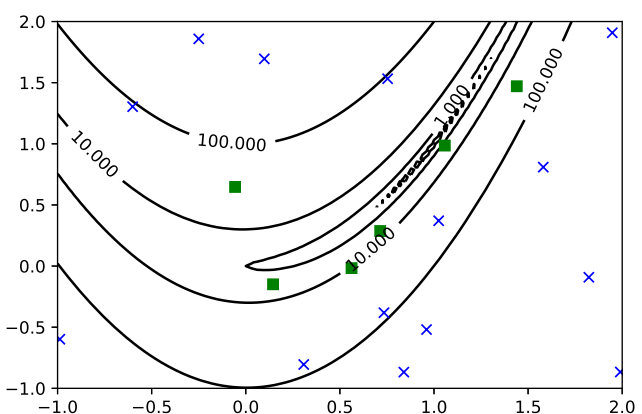

(b)

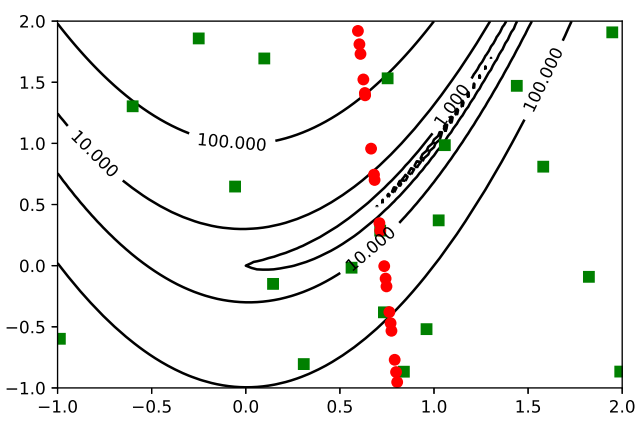

(d)

Figure 1: PCA and the valley landscape 
Next we identify the valley direction. Since the selected 6 points distribute along the valley, the valley direction can be regarded as a direction along which the variance of the 6 points is maximal. This direction can be identified by PCA. Assume that the valley direction is a linear line, the valley in fact can be approximated by the first principle component found by PCA. Let's project the 6 selected points onto the first principle component. Fig. 1c shows that the projected points (labeled by dotted points) approximately represent the valley direction.

But it should be pointed out if we apply PCA to the whole population and project all 20 points onto the first principle component, we cannot obtain the valley direction. Fig. $1 \mathrm{~d}$ shows that the mapped points (labeled by dotted points)) don't distribute along the valley direction. The mapped points could represent any direction if these 20 points are sampled at random.

\subsection{Proposed PCA Projection}

Based on the observation in the above subsection, we propose a new search operator. Here is our idea: Given a population, we select a group of points with smaller function values from the population and apply PCA to calculate principle components; then project the points onto the principle components; at the end, reconstruct the projected points in the original search space. These points are taken as the children. The procedure is described in detail as follows:

PCA-projection: Given a population $P$ and a fitness function $f(\mathbf{x})$,

1: Select $k$ individuals $\left\{\mathbf{x}_{1}, \cdots, \mathbf{x}_{k}\right\}$ with smaller fitness values from the population $P$ ( $k$ is a small constant). Denote these individuals by $\mathbf{X}$.

2: Calculate the $n \times 1$ mean vector $\mathbf{m}$ and $n \times n$ covariance matrix $\boldsymbol{\Sigma}$ :

$$
\mathbf{m}=\frac{1}{k} \sum_{i=1}^{k} \mathbf{x}_{i}, \quad \boldsymbol{\Sigma}=\frac{1}{k-1} \sum_{i=1}^{k}\left(\mathbf{x}_{i}-\mathbf{m}\right)\left(\mathbf{x}_{i}-\mathbf{m}\right)^{T} .
$$

3: Calculate the eigenvectors $\mathbf{v}_{1}, \cdots, \mathbf{v}_{n}$ of the covariance matrix $\boldsymbol{\Sigma}$, sorted them so that the eigenvalues of $\mathbf{v}_{i}$ is larger than $\mathbf{v}_{j}$ for $i<j$. Select the first $m$ eigenvectors $\mathbf{v}_{1}, \cdots, \mathbf{v}_{m}$ and form a $n \times m$ matrix $\mathbf{V}=\left[\mathbf{e}_{1}, \cdots, \mathbf{e}_{m}\right]$ where $m$ is a small constant (e.g., $m=5$ ).

4: Project $\mathbf{x}_{i}$ onto the $m$-dimensional space:

$$
\mathbf{y}_{i}=\mathbf{V}^{T}(\mathbf{x}-\mathbf{m}) .
$$

5: Reconstruct the projected point $\mathbf{x}_{i}$ in the original space:

$$
\mathbf{x}_{i}^{\prime}=\mathbf{m}+\mathbf{V} \mathbf{y}_{i}
$$

We call the search operator PCA-projection, rather than PCA-mutation [8], because it does not include a mutation step.

\subsection{Characteristics of PCA-projection}

PCA-projection is a nonlinear mapping from points in $\mathbb{R}^{n}$ to points in $\mathbb{R}^{m}$ which are assigned along the first $m$ principle components. It can be regarded as a multi-parent recombination operator. Like other recombination operators, it works only if the parent population keeps a degree of diversity. Otherwise, it might degenerate. For example, if $k$ points are identical (say $\mathbf{x}$ ), then after PCA-projection, the projected points are still $\mathbf{x}$. If $k$ points distribute on the same line (say $\mathbf{y}=\mathbf{a x}+\mathbf{b}$ ), then the first principle component is $\mathbf{y}=\mathbf{a x}+\mathbf{b}$. After PCA-projection, there is no change on these $k$ points.

PCA-projection generates the direction along which the distribution of points has the maximal variance. It is not the gradient direction. Let's show the difference through a simple example. Consider the minimisation problem

$$
\min f(x, y)=|x|+0 \times y .
$$

If $k$ points distribute on the same line ( $\operatorname{say} x_{i}=1, y_{i}=i$, where $i=1, \cdots, k$ ), then the first component direction found by PCA-projection is $x=1$. But the gradient direction is perpendicular to the line $x=1$.

Compared with PCA-mutation in [8], PCA-projection has three new characteristics: 
1. The time complexity of PCA-projection is less than PCA-mutation in [8]. Given $k$ points in $\mathbb{R}^{n}$, the covariance matrix computation in PCA is $O\left(k^{2} n\right)$ and its eigenvalue decomposition is $O\left(n^{3}\right)$. So, the complexity of PCA is $O\left(k^{2} n+n^{3}\right)$. In PCA-projection, only $k$ good points are sampled from the population. For example, $k=8$ in this paper, so, its time complexity is $O\left(8^{2} n+n^{3}\right)$. But in PCAmutation, the number of points equals to the population size. For example, population size is $12 n$, so, its time complexity is $O\left(144 n^{3}+n^{3}\right)$.

2. The PCA-projection has an intuitive explanation. Given a valley landscape, it projects an individual to a new position along the valley direction.

3. It also takes the advantage of compressing a higher dimensional data into a lower dimension space. It projects a point into a lower dimensional space.

\section{Two New Algorithms Enhanced by PCA-Projection}

In this section, we present two MOEAs enhanced with PCA-projection for COPs.

\subsection{New Algorithm 1: $P M O D E=C M O D E+P C A$-projection}

We design the first algorithm though adding PCA-projection to CMODE, an algorithm proposed by Cai and Wang [21, 22]. It combines multiobjective optimization with differential evolution. CMODE is used to solve the standard bi-objective problem:

$$
\min (f(\mathbf{x}), v(\mathbf{x})), \quad x \in \Omega .
$$

CMODE belongs to the family of MOEAs based on non-dominance. At each generation, it identifies non-dominant solutions under functions $(f, v)$ and replaces those dominated solutions. It is straightforward to add PCA-projection into CMODE through the mixed strategy, that is to apply PCA-projection with probability $p$ and normal mutation and crossover operations with probability $1-p$. After PCA-projection is added into CMODE, we name the new algorithm PMODE. The pseudo-code of PMODE is shown as below.

1: initialise a population $P$ of solutions at random;

2: evaluate the fitness value $f$ and constraint violation $v$ for each individual in $P$;

3: set $F E S=\mu$;

4: set $A=\emptyset$;

5: for $t=1, \cdots, F E S_{\max }$ do

initialise children population $C=\emptyset$; randomly choose $\lambda$ individuals (denoted by $Q$ ) from population $P$; let $P^{\prime}=P \backslash Q$;

for $\mathbf{x}_{i}$ in $Q, i=1, \ldots, \lambda$ do

if rand $<0.1$ then perform PCA-projection on set $Q$ and generate $\lambda$ projected solutions $\mathbf{x}_{1}^{\prime}, \cdots, x_{\lambda}^{\prime}$; let $\mathbf{y}_{i}=\mathbf{x}_{i}^{\prime}$; else

generate a trail vector $\mathbf{y}_{i}$ by mutation and crossover;

end if

update $C \leftarrow C \cup\left\{\mathbf{y}_{i}\right\}$;

end for

evaluate the values of $f$ and $v$ for each individual in $C$; and update $F E S \leftarrow F E S+\lambda$; identify all non-dominated individuals in $C$ (denoted by $R$ );

for each individual $\mathrm{x}$ in $R$ do find all individual(s) in $Q$ dominated by $\mathbf{x}$;

randomly replace one of these dominated individuals by $\mathbf{x}$; end for

let $P=P^{\prime} \cup Q$; 
25: $\quad$ if no feasible solution exists in $R$ then

26: $\quad$ identify the infeasible solution $\mathbf{x}$ in $R$ with the lowest degree of constraint violation and add it to archive $A$;

27: $\quad$ end if

28: $\quad$ if $\bmod (t, k)=0$ then

29: $\quad$ execute the infeasible solution replacement mechanism and set $A=\emptyset$;

30: $\quad$ end if

31: end for

Output: the best found solution

For the sake of self-contained presentation, we explain PMODE in detail. Lines 1-4 are initialisation steps. In lines 1 and 2 , an initial population $P$ is generated at random, in which all initial vectors are chosen randomly from $\left[L_{i}, U_{i}\right]^{n}$. Then the fitness value and constraint violation degree of each individual are evaluated. In line $3, F E S$ records the number of fitness evaluations. In line $4, A$ is an archive to store the infeasible individual with the lowest degree of constraint violation. Initially it is set to an empty set.

Lines 5 to 31 are the main loop of this algorithm. $F E S_{\max }$ represents the maximum number of function evaluations. In line $6, C$ is a set to keep the children generated by PCA-projection, mutation and crossover. Initially it is set to an empty set.

In lines 7 and $8, \lambda$ individuals (denoted by $Q$ ) are selected from population $P . P^{\prime}$ denotes the rest individuals in $P$. Subpopulation $Q$ takes part in PCA-projection, mutation and crossover, but subpopulation $P^{\prime}$ does not.

In lines 10 to 15 , a trial vector $y$ is generated by a mixed strategy. PCA-projection is chosen with probability 0.1 , or mutation and crossover are chosen with probability 0.9. rand is a random number in $[0,1]$. This is the main difference form CMODE [22] in which PCA-projection exists.

In line 11, PCA-projection is conducted on subpopulation $Q$, then $\lambda$ new solutions are generated. The procedure of PCA-projection on $Q$ is given as follows: (1) calculate the 5 eigenvectors $\mathbf{v}_{1}, \cdots, \mathbf{v}_{n}$ of the covariance matrix with the largest eigenvalues; (2) project $\mathbf{x}_{i}$ onto a lower-dimensional space; (3) reconstruct the projected point $\mathbf{x}_{i}$ in the original space, denoted by $x_{i}^{\prime}$. In line 12 , set the trial vector $\mathbf{y}_{i}=\mathbf{x}_{i}^{\prime}$.

In line 14 , generate a mutant vector $\mathbf{v}_{i}$ by

$$
\mathbf{v}_{i}=\mathbf{x}_{r 1}+F \cdot\left(\mathbf{x}_{r 2}-\mathbf{x}_{r 3}\right)
$$

where random indexes $r 1, r 2, r 3$ are mutually different integers. They are also chosen to be different from the running index $i . F$ is a real and constant factor from $[0,2]$ which controls the amplification of the differential variation $\left(\mathbf{x}_{r 2}-\mathbf{x}_{r 3}\right)$.

Then the trial vector $\mathbf{y}_{i}$ is generated by crossover on the mutant vector $\mathbf{v}_{\mathbf{i}}$. In more detail, trial vector $\mathbf{y}_{i}=\left(\mathbf{y}_{i, 1}, \cdots, \mathbf{y}_{i, n}\right)$ is constructed as follows:

$$
y_{i, j}=\left\{\begin{array}{l}
\mathbf{v}_{i, j}, \quad \text { if } \operatorname{rand}_{j}(1,0) \leq C R \text { or } j=j_{\text {rand }}, \\
\mathbf{x}_{i, j}, \quad \text { otherwise, }
\end{array} \quad j=1, \cdots, n,\right.
$$

where $\operatorname{rand}_{j}(0,1)$ is a uniform random number from $[0,1]$. Index $j_{\text {rand }}$ is randomly chosen from $\{1, \cdots, n\}$. $C R \in[0,1]$ denotes the crossover constant which has to be determined by the user. In addition, the condition " $j=j_{\text {rand }}$ is used to ensure the trial vector $\mathbf{y}_{i}$ gets at least one parameter from vector $\mathbf{v}_{\mathbf{i}}$.

In line 16 , the trail vector $\mathbf{y}_{i}$ is added into children subpopulation $C$.

In line 18, the values of $f$ and $v$ of each individual in $C$ are evaluated. Then in line 19, all non-dominated solutions in $C$ are identified and stored in set $R$. In lines 21 and 22, non-dominated individuals in $R$ replace dominated individuals in $Q$ at random.

In line 24, a new generation population $P$ is generated by merging $P^{\prime} \cup Q$.

Lines 25 to 30 are the infeasible solution replacement mechanism, which is the same as CMODE [22].

The difference between PMODE and CMODE is PCA-projection. There is no other change in other parts. This design aims to evaluate the effectiveness of PCA-project without the interface of other factors. 


\subsection{New Algorithm 2: HECO-PDE $=H E C O-D E+P C A$-projection}

We design the second algorithm through adding PCA-projection to HECO-DE ${ }^{1}$, an algorithm proposed by $\mathrm{Xu}, \mathrm{He}$ and Shang under the framework of helper and equivalent objectives. HECO-DE is built upon a single-objective EA (LSHADE44) for constrained optimisation [33], but aims at minimising two objective functions within population $P$ :

$$
\min \mathbf{f}(\mathbf{x})=(e(\mathbf{x}), f(\mathbf{x})), \quad \mathbf{x} \in P .
$$

This bi-objective optimisation problem is different from the standard one (21) because function $e(\mathbf{x})$ is given by

$$
e(\mathbf{x})=w_{1} \tilde{e}(\mathbf{x})+w_{2} v(\mathbf{x})
$$

where $w_{1}, w_{2} \geq 0$ are weights. Function $\tilde{e}(\mathbf{x})=\left|f(\mathbf{x})-f^{*}(P)\right|$ in which $f^{*}(P)$ denotes the best individual's fitness in population $P$, given as

$$
f^{*}(P)= \begin{cases}\min \{v(\mathbf{x}) ; \mathbf{x} \in P\}, & \text { if } P \cap \Omega_{F}=\emptyset, \\ \min \left\{f(\mathbf{x}) ; \mathbf{x} \in P \cap \Omega_{F}\right\}, & \text { if } P \cap \Omega_{F} \neq \emptyset .\end{cases}
$$

HECO-DE belongs to the family of MOEAs based on decomposition. Problem (24) is decomposed into $\lambda$ single objective subproblems through the weighted sum method: for $i=1, \cdots, \lambda$,

$$
\min f_{i}(\mathbf{x})=w_{1 i} \tilde{e}(\mathbf{x})+w_{2 i} v(\mathbf{x})+w_{3 i} f(\mathbf{x}),
$$

where $w_{1 i}, w_{2 i}, w_{3 i} \geq 0$ are $\lambda$ tuples of weights.

An extra term $\tilde{e}$ is added besides the original objective function $f$ and constraint violation degree $v$, so problem (26) is equivalent to a tri-objective optimisation problem.

$$
\min \mathbf{f}(\mathbf{x})=(\tilde{e}(\mathbf{x}), f(\mathbf{x}), v(\mathbf{x})), \quad \mathbf{x} \in P .
$$

PCA-projection is added into HECO-DE through a mixed strategy, that is to apply PCA-projection with probability $p$ and normal mutation and crossover operations with probability $1-p$. For the new algorithm enhanced with PCA-projection, we call it HECO-PDE. The pseudo-code of HECO-PDE is shown as follows.

Input: objective function $f(\mathbf{x})$ and constraint violation $v(\mathbf{x})$;

1: initialise algorithm parameters: the required sizes $\mu_{0}, \mu_{T_{\max }}$; circle memories $M_{F}$ and $M_{C R}$, memory size $H$; probabilities $q_{k}=1 / 4, k=1, \cdots, 4$, archive $A=\emptyset$;

2: $t=0$;

3: randomly generate an initial population $P$ of size $\mu_{0}$;

4: evaluate $f(\mathbf{x})$ and $v(\mathbf{x})$ for $\mathbf{x} \in P$;

5: $F E S=\mu_{0}$;

6: while $F E S \leq F E S_{\max }\left(\right.$ or $\left.t \leq T_{\max }\right)$ do

7: $\quad$ adjust weights;

8: $\quad$ for each strategy, set $S_{F}=\emptyset, S_{C R}=\emptyset$;

9: $\quad$ children population $C=\emptyset$;

10: $\quad$ randomly select $\lambda$ individuals (denoted by $Q$ ) from $P$ and then set $P^{\prime}=P \backslash Q$;

for $x_{i}$ in $Q, i=1, \ldots, \lambda$ do

if rand $<0.1$ then perform PCA-projection on set $Q$, and generate $\lambda$ projected individuals $\left(\mathbf{x}_{1}^{\prime}, \cdots, x_{\lambda}^{\prime}\right)$; let trail vector $\mathbf{y}_{i}=\mathbf{x}_{i}^{\prime}$;

else

$$
\text { choose one strategy subject to probabilities } q_{k}, k=1, \cdots, 4 \text { and generate parameters } F \text { and }
$$
$C R$ with respective circle memories;

\footnotetext{
${ }^{1}$ Xu, T., He, J.,\& Shang, C. (2019). Helper and Equivalent Objectives: An Efficient Approach to Constrained Optimisation. arXiv preprint arXiv:1903.04886. Also in ACM GECCO 2019 Competition in Constrained Optimisation.
} 


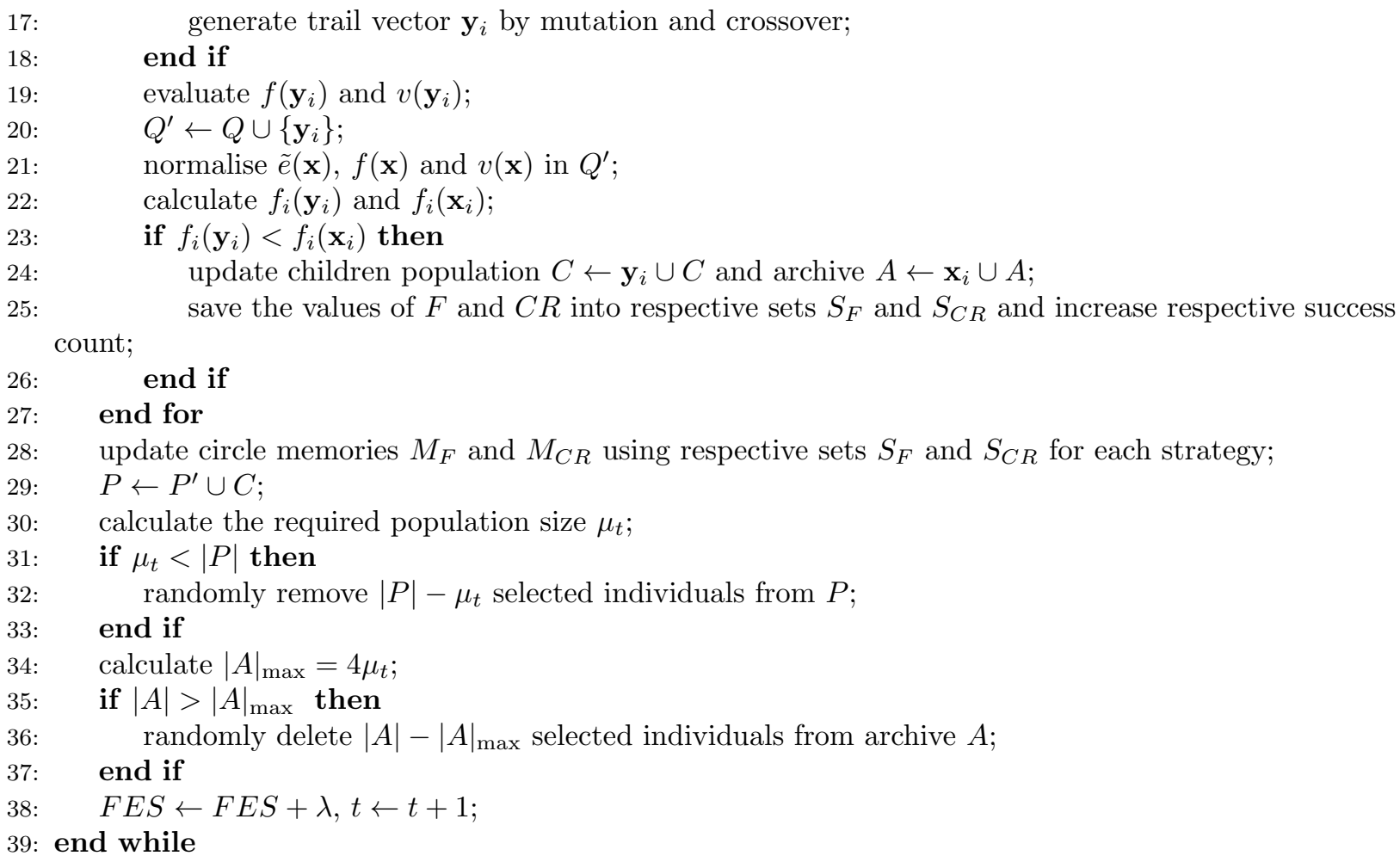

Output: the best individual $\mathrm{x} \in P$.

For the sake of self-contained presentation, we explain HECO-PDE in detail. Lines 1 and 2 are to initialise several algorithm parameters, including:

1. $\mu_{0}$ is the required initial population size and $\mu_{T_{\max }}$ is final size;

2. FES $S_{\max }$ is a pre-defined maximum number of fitness evaluations;

3. $F$ and $C R$ are two circle memories for parameters;

4. $H$ is the size of historical memories;

5. $q_{k}, k=1, \cdots, 4$ are the initial probability distribution of four strategies

6. $A$ is external archive;

7. $t$ is the number of generations.

Lines 3-5 are to generate an initial population $P$ at random and evaluate the objective value $f(\mathbf{x})$ and constraint violation $v(\mathbf{x})$ for each individual $\mathbf{x}$. The number of fitness evaluation $F E S=\mu_{0}$.

The while loop in lines 6-39 is the main part of the algorithm.

In line 7 , weights on functions $\tilde{e}, v, f$ are adjusted by the following formulas:

$$
\begin{aligned}
& w_{1 i, t}=\left(\frac{t}{T_{\max }}\right)^{20 \times i} . \\
& w_{2 i, t}=\frac{i}{\lambda}\left(\frac{t}{T_{\max }}\right)^{\frac{5 \times i}{\lambda}} . \\
& w_{3 i, t}=\left(1-\frac{i}{\lambda}\right)\left(1-\frac{t}{T_{\max }}\right)^{\frac{5 \times i}{\lambda}} .
\end{aligned}
$$

Weights $w_{1 i, t}$ and $w_{2 i, t}$ increase over $t$ but $w_{3 i, t}$ decreases over $t$.

In line 8 , sets $S_{F}$ and $S_{C R}$ are assigned to an empty set for each strategy. Here a strategy means a combination of a mutation operator and a crossover operator. Sets $S_{F}$ and $S_{C R}$ are used to preserve successful values of $F$ in mutation and $C R$ in crossover for each search strategy respectively.

In line 9 , set $C$ (used for saving children population) is also assigned to an empty set. 
In line 10, $\lambda$ individuals (denoted as $Q$ ) are selected randomly from $P$ without putting them back to $P$. Since calculating the value of the equivalent function (25) relies on ranking individuals in $Q$, its size $\lambda$ is set to a small constant. Thus, the time complexity of ranking is a constant. This is different from LSHADE44 [33] which applies a search strategy to population $P$. The time complexity of ranking in LSHADE44 [33] is a function of $n$.

In lines 12-18, a trial vector $y$ is generated by a mixed strategy. PCA-projection is chosen with probability 0.1 , or mutation and crossover are chosen with probability 0.9 . rand is a random number in $[0,1]$.

In lines 13 and 14, PCA-projection is conducted on $Q$. Then $\lambda$ projected vectors are generated, denoted by $\mathbf{x}_{i}^{\prime}, i=1, \cdots, \lambda$. Let $y_{i}=x_{i}^{\prime}$.

In line 16 , the $k$ th search strategy is selected with probability $q_{k}$. A mechanism of competition of strategies $[34,35]$ is employed to create trial points. The probability $q_{k}$ is adapted according to its success counts. A used strategy is considered successful if a generated trial point $y$ is better than the original point $x$. The selection probability

$$
q_{k}=\frac{n_{k}+n_{0}}{\sum_{i=1}^{4}\left(n_{i}+n_{0}\right)},
$$

where $n_{k}$ is the count of the $k$ th strategy's successes, and $n_{0}>0$ is a constant. Parameters $F$ and $C R$ are generated from respective circle memories.

Then trial vector $\mathbf{y}_{i}$ is generated by a strategy, i.e., mutation plus crossover. There are two mutation operators used in the algorithm. The first one is current-to-pbest/1 mutation as proposed in JADE [36].

$$
\mathbf{u}_{i}=\mathbf{x}_{i}+F\left(\mathbf{x}_{\text {pbest }}-\mathbf{x}_{i}\right)+F\left(\mathbf{x}_{r_{1}}-\mathbf{x}_{r_{2}}\right) \text {, }
$$

where $\mathbf{x}_{i}$ is the target point. $\mathbf{x}_{\text {pbest }}$ is randomly chosen from the top $100 p \%$ of population $P$ where $p \in(0,1]$ a parameter. $\mathbf{x}_{r_{1}}$ is uniformly chosen from population $P$. Individual $\mathbf{x}_{r_{2}}$ is randomly selected from $P \cup A$ where $A$ is an archive. $F$ is a mutation factor.

The second mutation is randrl/1 mutation [37].

$$
\begin{aligned}
\mathbf{u}_{i} & =\mathbf{x}_{r_{1}}+F\left(\mathbf{x}_{r_{2}}-\mathbf{x}_{r_{3}}\right), \\
\mathbf{u}_{i} & =\mathbf{x}_{r_{1}^{*}}+F\left(\mathbf{x}_{r_{2}^{*}}-\mathbf{x}_{r_{3}^{*}}\right) .
\end{aligned}
$$

In mutation (31), $\mathbf{x}_{r_{1}}, \mathbf{x}_{r_{2}}$ and $\mathbf{x}_{r_{3}}$ are randomly chosen from population $P$. These three points are mutually distinct and different from $\mathbf{x}_{i}$. In mutation (32), $\mathbf{x}_{r_{1}}, \mathbf{x}_{r_{2}}$ and $\mathbf{x}_{r_{3}}$ are selected as mutation (31) and then are ranked. $\mathbf{x}_{r_{1}^{*}}$ is the best individual while $\mathbf{x}_{r_{2}^{*}}$ and $\mathbf{x}_{r_{3}^{*}}$ denote the rest two. $F_{k}(k=1, \cdots, K)$ is the mutation factor. Kaelo and Ali [37] tested the randrl/1 mutation on a set of 50 difficult benchmark problems, with the results indicating that the use of this strategy speeds up the search by $30 \%$ without significant decreasing the reliability as compared to searching with the original mutation operator.

There are two crossover operators used in the algorithm. The first one is binomial crossover: It combines coordinates of $\mathbf{x}_{i}$ with coordinates of mutant $\mathbf{u}_{i}$ into a trial point $\mathbf{y}_{i}$

$$
y_{i, j}= \begin{cases}u_{i, j}, & \text { if } \operatorname{rand}_{j}(0,1) \leq C R \text { or } j=j_{\text {rand }}, \\ x_{i, j}, & \text { otherwise, }\end{cases}
$$

where the index $j_{\text {rand }}$ is a randomly chosen integer from $[1, D] \cdot \operatorname{rand}_{j}(0,1)$ is a uniform random number. $C R \in[0,1]$ is a crossover control parameter.

The second crossover is the exponential crossover [32]. It combines the coordinates of $\mathbf{x}_{i}$ with the coordinates of the mutant $\mathbf{u}_{i}$ into a trial point $\mathbf{y}_{i}$ as follows:

$$
y_{i, j}= \begin{cases}u_{i, j}, & j=\langle l\rangle_{D}, \ldots,\langle l+L-1\rangle_{D}, \\ x_{i, j}, & \text { otherwise }\end{cases}
$$

where \langle\rangle$_{D}$ denotes a modulo function with modulus D. $l$ is the starting integer number randomly chosen from $[0, D-1]$, and the integer $L$ is drawn from $[0, D-1]$ subject to an exponential probability distribution. Thus, four search strategies (combinations) can be produced. 
In line $20, \mathbf{y}_{i}$ is appended to subpopulation $Q$, which results in an enlarged subpopulation $Q^{\prime}$. In line 21 , the values of $\tilde{e}(\mathbf{x}), f(\mathbf{x})$ and $v(\mathbf{x})$ normalised by the max-min normalisation for each individual $\mathbf{x}$ in $Q^{\prime}$. Then the values of $f_{i}\left(\mathbf{y}_{i}\right)$ and $f_{i}\left(\mathbf{x}_{i}\right)$ are calculated according to formula (26). If $f_{i}\left(\mathbf{y}_{i}\right)<f_{i}\left(\mathbf{x}_{i}\right)$, i.e., the trial point is better than its parent in terms of $f_{i}$, then in line $24, \mathbf{x}_{i}$ is saved into archive $A$, and $\mathbf{y}_{i}$ into children set $C$. The $i$ th individual in subpopulation $Q$ is used to minimise $f_{i}$. HECO-DE minimises $\lambda$ objective functions $f_{i}$ simultaneously.

In line 25, parameters $F$ in mutation and $C R$ in crossover are saved into respective sets $S_{F}$ and $S_{C R}$. Then the respective success counter is updated.

After completing search on subpopulation $Q$, in lines 28, circle memories $M_{F}$ and $M_{C R}$ are updated using respective $S_{F}$ and $S_{C R}$ for each strategy. The detail of this process refers to LSHADE44 [33].

In line 29, population $P$ is updated by merging children $C$ and those individuals not involved in search. In line 30-33, if population size $|P|$ is greater than the required size $\mu_{t}$, then $|P|-\mu_{t}$ non-best individuals are removed from $P$ at random where $N_{t}$ is given by

$$
\mu_{t}=\operatorname{round}\left(\mu_{0}-\frac{t}{T_{\max }}\left(\mu_{0}-\mu_{T_{\max }}\right)\right) .
$$

Similarly in lines 34-37, if archive size $|A|$ is greater than the maximum size $|A|_{\max }$, then $|A|-|A|_{\max }$ individuals are removed from $A$ at random.

Except PCA-projection, the other part of HECO-PDE is the same as HECO-DE. This design aims to evaluate the effectiveness of PCA-project without the interface of other factors.

\section{Comparative Experiments and Results}

In order to demonstrate the effectiveness of PCA-projection, HECO-PDE and PMODE are tested on the IEEE CEC 2017 benchmark suite in constrained optimization competition [17] and compared with the state-of-art EAs participated in the CEC 2018 competition [18].

\subsection{The CEC 2017 Benchmark Suit}

The IEEE CEC 2017 benchmark suit in constrained optimisation competition [18] consists of 28 problems with the dimension $D=10,30,50,100$, therefore, $4 \times 28$ instances in total. This suit was adopted by both CEC 2017 and 2018 competitions [17]. The detail of these problems are listed in Table 1.

\subsection{Experimental Settings}

As suggested by the CEC 2018 competition [18], the maximum number of function evaluations $F E S_{\max }=$ $20000 D$. For each algorithm, 25 independent runs were taken on each problem and dimension respectively.

The parameters of PMODE are set as follows. For a fair comparison, the setting is chosen as the same as that used in CMODE although fine-tuning parameters of PMODE may lead to better results.

1. population size $\mu$ is set as 180 ;

2. scaling factor $F$ is randomly chosen between 0.5 and 0.6 ;

3. PCA crossover controlling parameter $C R$ is randomly chosen between 0.9 and 0.95 ;

4. subpopulation size $\lambda=8$;

5 . the interval of executing infeasible solution replacement $k=22$.

The parameters of HECO-PDE are set as follows. Although fine-tuning parameters of HECO-PDE may lead to better results, the setting is chosen as the same as that used in HECO-DE for a fair comparison.

1. the number of subproblem $Q \lambda=12$;

2 . in strategy competition, $n_{0}=2, K=4, \delta=1 / 20$;

3. the size of historical memories $H=5$;

4. the initial and final required population sizes $\mu_{0}=12 \times D, \mu_{T_{\max }}=\lambda$. 
Table 1: Details of 28 test problems. $I$ is the number of inequality constraints, $E$ is the number of equality constraints

\begin{tabular}{|c|c|c|c|}
\hline \multirow{2}{*}{$\begin{array}{c}\text { Problem } \\
\text { Search Range }\end{array}$} & \multirow{2}{*}{ Type of Objective } & \multicolumn{2}{|c|}{ Number of Constraints } \\
\hline & & $E$ & $I$ \\
\hline $\begin{array}{c}\mathrm{C} 01 \\
{[-100,100]^{D}}\end{array}$ & Non Separable & 0 & $\begin{array}{c}1 \\
\text { Separable } \\
\end{array}$ \\
\hline $\begin{array}{c}\mathrm{C} 02 \\
{[-100,100]^{D}}\end{array}$ & $\begin{array}{c}\text { Non Separable, } \\
\text { Rotated }\end{array}$ & 0 & $\begin{array}{c}1 \\
\text { Non Separable, Rotated }\end{array}$ \\
\hline $\begin{array}{c}\mathrm{C} 03 \\
{[-100,100]^{D}}\end{array}$ & Non Separable & $\begin{array}{c}1 \\
\text { Separable }\end{array}$ & $\begin{array}{c}1 \\
\text { Separable }\end{array}$ \\
\hline $\begin{array}{c}\mathrm{C} 04 \\
{[-10,10]^{D}}\end{array}$ & Separable & 0 & $\begin{array}{c}2 \\
\text { Separable }\end{array}$ \\
\hline $\begin{array}{c}\mathrm{C} 05 \\
{[-10,10]^{D}}\end{array}$ & Non Separable & 0 & $\begin{array}{c}2 \\
\text { Non Separable, Rotated }\end{array}$ \\
\hline $\begin{array}{c}\mathrm{C} 06 \\
{[-20,20]^{D}}\end{array}$ & Separable & 6 & $\begin{array}{c}0 \\
\text { Separable }\end{array}$ \\
\hline $\begin{array}{c}\mathrm{C} 07 \\
{[-50,50]^{D}}\end{array}$ & Separable & $\begin{array}{c}2 \\
\text { Separable }\end{array}$ & 0 \\
\hline $\begin{array}{c}\mathrm{C} 08 \\
{[-100,100]^{D}}\end{array}$ & Separable & $\begin{array}{c}2 \\
\text { Non Separable }\end{array}$ & 0 \\
\hline $\begin{array}{c}\mathrm{C} 09 \\
{[-10,10]^{D}}\end{array}$ & Separable & $\begin{array}{c}2 \\
\text { Non Separable } \\
\end{array}$ & 0 \\
\hline $\begin{array}{c}\mathrm{C} 10 \\
{[-100,100]^{D}}\end{array}$ & Separable & $\begin{array}{c}2 \\
\text { Non Separable } \\
\end{array}$ & 0 \\
\hline $\begin{array}{c}\mathrm{C} 11 \\
{[-100,100]^{D}}\end{array}$ & Separable & $\begin{array}{c}1 \\
\text { Non Separable } \\
\end{array}$ & $\begin{array}{c}1 \\
\text { Non Separable } \\
\end{array}$ \\
\hline $\begin{array}{c}\mathrm{C} 12 \\
{[-100,100]^{D}}\end{array}$ & Separable & 0 & $\begin{array}{c}2 \\
\text { Separable }\end{array}$ \\
\hline $\begin{array}{c}\mathrm{C} 13 \\
{[-100,100]^{D}}\end{array}$ & Non Separable & 0 & $\begin{array}{c}3 \\
\text { Separable }\end{array}$ \\
\hline $\begin{array}{c}\mathrm{C} 14 \\
{[-100,100]^{D}}\end{array}$ & Non Separable & $\begin{array}{c}1 \\
\text { Separable }\end{array}$ & $\begin{array}{c}1 \\
\text { Separable }\end{array}$ \\
\hline $\begin{array}{c}\mathrm{C} 15 \\
{[-100,100]^{D}}\end{array}$ & Separable & 1 & 1 \\
\hline $\begin{array}{c}\mathrm{C} 16 \\
{[-100,100]^{D}}\end{array}$ & Separable & $\begin{array}{c}1 \\
\text { Non Separable }\end{array}$ & $\begin{array}{c}1 \\
\text { Separable }\end{array}$ \\
\hline $\begin{array}{c}\mathrm{C} 17 \\
{[-100,100]^{D}}\end{array}$ & Non Separable & $\begin{array}{c}1 \\
\text { Non Separable }\end{array}$ & $\begin{array}{c}1 \\
\text { Separable }\end{array}$ \\
\hline $\begin{array}{c}\mathrm{C} 18 \\
{[-100,100]^{D}}\end{array}$ & Separable & 1 & 2 \\
\hline $\begin{array}{c}\mathrm{C} 19 \\
{[-50,50]^{D}}\end{array}$ & Separable & 0 & $\begin{array}{c}2 \\
\text { Non Separable }\end{array}$ \\
\hline $\begin{array}{c}\mathrm{C} 20 \\
{[-100,100]^{D}}\end{array}$ & Non Separable & 0 & 2 \\
\hline $\begin{array}{c}\mathrm{C} 21 \\
{[-100,100]^{D}}\end{array}$ & Rotated & 0 & $\begin{array}{c}2 \\
\text { Rotated }\end{array}$ \\
\hline $\begin{array}{c}\mathrm{C} 22 \\
{[-100,100]^{D}}\end{array}$ & Rotated & 0 & $\begin{array}{c}3 \\
\text { Rotated }\end{array}$ \\
\hline $\begin{array}{c}\mathrm{C} 23 \\
{[-100,100]^{D}}\end{array}$ & Rotated & $\begin{array}{c}1 \\
\text { Rotated }\end{array}$ & $\begin{array}{c}1 \\
\text { Rotated }\end{array}$ \\
\hline $\begin{array}{c}\mathrm{C} 24 \\
{[-100,100]^{D}}\end{array}$ & Rotated & $\begin{array}{c}1 \\
\text { Rotated }\end{array}$ & $\begin{array}{c}1 \\
\text { Rotated }\end{array}$ \\
\hline $\begin{array}{c}\mathrm{C} 25 \\
{[-100,100]^{D}}\end{array}$ & Rotated & $\begin{array}{c}1 \\
\text { Rotated }\end{array}$ & $\begin{array}{c}1 \\
\text { Rotated }\end{array}$ \\
\hline $\begin{array}{c}\mathrm{C} 26 \\
{[-100,100]^{D}}\end{array}$ & Rotated & $\begin{array}{c}1 \\
\text { Rotated }\end{array}$ & $\begin{array}{c}1 \\
\text { Rotated }\end{array}$ \\
\hline $\begin{array}{c}\mathrm{C} 27 \\
{[-100,100]^{D}}\end{array}$ & Rotated & $\begin{array}{c}1 \\
\text { Rotated }\end{array}$ & $\begin{array}{c}2 \\
\text { Rotated }\end{array}$ \\
\hline $\begin{array}{c}\mathrm{C} 28 \\
{[-50,50]^{D}}\end{array}$ & Rotated & 0 & $\begin{array}{c}2 \\
\text { Rotated }\end{array}$ \\
\hline
\end{tabular}




\subsection{Description of EAs under Comparison}

HECO-PDE and PMODE are compared with all seven EAs participated in the CEC 2018 constrained optimisation competition [18] apart from HECO-DE and CMODE. All EAs from the CEC 2017 competition belong to the single-objective method for COPs. Thus, a latest decomposition-based multi-objective EA, DeCODE [29], is also taken in the comparison. These algorithms are described as follows.

1. CAL-SHADE [38]: Success-History based Adaptive Differential Evolution Algorithm including liner population size reduction, enhanced with adaptive constraint violation handling, i.e. adaptive $\epsilon$ constraint handling.

2. LSHADE+IDE [39]: A simple framework for cooperation of two advanced adaptive DE variants. The search process is divided into two stages: (i) search feasible solutions via minimizing the mean violation and stopped if a number of feasible solutions are found. (ii) minimize the function value until the stop condition is reached.

3. LSHADE44 [33]: Success-History based Adaptive Differential Evolution Algorithm including liner population size reduction, uses three different additional strategies compete, with Deb's superiority of feasibility rule.

4. UDE [40]: Uses three trial vector generation strategies and two parameter settings. At each generation, UDE divides the current population into two sub-populations. In the first population, UDE employs all the three trial vector generation strategies on each target vector. For another one, UDE employs strategy adaption from learning experience from evolution in first population.

5. MA-ES [41]: Combines the Matrix Adaptation Evolution Strategy for unconstrained optimization with well-known constraint handling techniques. It handles box-constraints by reflecting exceeding components into the predefined box. Additional in-/equality constraints are dealt with by application of two constraint handling techniques: $\epsilon$-level ordering and a repair step that is based on gradient approximation.

6. IUDE [42]: An improved version of UDE. Different from UDE, local search and duplication operators have been removed, it employs a combination of $\epsilon$-constraint handling technique and Deb's superiority of feasibility rule.

7. LSHADE-IEpsilon [43]: An improved $\epsilon$-constrained handling method (IEpsilon) for solving constrained single-objective optimization problems. The IEpsilon method adaptively adjusts the value of $\epsilon$ according to the proportion of feasible solutions in the current population. Furthermore, a new mutation operator $\mathrm{DE} / \mathrm{randr} 1^{*} / 1$ is proposed.

8. DeCODE [29]: A recent decomposition-based EA made use of the weighted sum approach to decompose the transformed bi-objective problem into a number of scalar optimisation subproblems and then applied differential evolution to solve them. They designed a strategy of adjusting weights and a restart strategy to tackle COPs with complicated constraints.

\subsection{The Rules for Ranking Algorithms}

Because the CEC 2017 competition aimed to compare performance of a group of EAs together, a set of rules was provided for ranking all algorithms [17]. Our comparison follows the same rules, which are listed as below.

1. The procedure for ranking algorithms based on mean values:

(a) Rank the algorithms based on feasibility rate;

(b) Then rank the algorithms according to the mean violation amounts;

(c) At last, rank the algorithms in terms of mean objective function value.

2. The procedure for ranking the algorithms based on the median solutions:

(a) A feasible solution is better than an infeasible solution;

(b) Rank feasible solutions based on their objective function values;

(c) Rank infeasible solutions according to their constraint violation amounts. 
3. Ranking all algorithms on multiple problems: for each problem, algorithms' ranks are determined in terms of the mean values and median solutions at maximum allowed number of evaluations, respectively. The total rank value of an algorithm is calculated as below:

$$
\text { Rank value }=\sum_{i=1}^{28} r a n k_{i}(\text { using mean value })+\sum_{i=1}^{28} r a n k_{i}(\text { using median solution })
$$

\subsection{Comparative Experimental Results}

Table 2 summarises ranks of all algorithms on four dimensions and total ranks. HECO-PDE and PMODE got lower rank values than HECO-DE and CMODE, respectively. This result clearly demonstrates that HECO-DE and CMODE are improved by PCA-projection. Moreover, HECO-PDE got the lowest rank value among all compared algorithms. This result means that HECO-PDE enhanced with PCA-projection is the best in terms of the overall performance.

Table 2: Total ranks of CMODE, PMODE, HECO-DE, HECO-PDE, DeCODE and seven EAs in CEC2018 competition

\begin{tabular}{|lccccc|}
\hline Algorithm/Dimension & $10 D$ & $30 D$ & $50 D$ & $100 D$ & Total \\
\hline CAL_LSAHDE(2017) & 418 & 398 & 428 & 435 & 1679 \\
LSHADE44+IDE(2017) & 299 & 365 & 385 & 353 & 1402 \\
LSAHDE44(2017) & 319 & 313 & 308 & 310 & 1250 \\
UDE(2017) & 330 & 344 & 345 & 390 & 1409 \\
MA_ES(2018) & 266 & 240 & 243 & 246 & 995 \\
IUDE(2018) & 193 & 226 & 224 & 292 & 935 \\
LSAHDE_IEpsilon(2018) & 199 & 246 & 292 & 333 & 1070 \\
DeCODE & 237 & 276 & 277 & 296 & 1086 \\
CMODE & 443 & 618 & 628 & 631 & 2320 \\
PMODE & 425 & 610 & 630 & 626 & 2291 \\
HECO-DE & 173 & 164 & 177 & 183 & 697 \\
HECO-PDE & 155 & 138 & 152 & 177 & 622 \\
\hline
\end{tabular}

The detailed rank values of all algorithms on mean values and median solutions on 28 test problems with the dimension of 10D, 30D, 50D and 100D are shown in Table 3-10, respectively.

Regarding the test functions with $10 D$, rank values based on mean values and median solution on the 28 test functions are reported in Table 3 and 4, respectively. As shown in Table 3, in terms of mean values with 10D, HECO-DE and CMODE got total rank values, with 81 and 236 respectively. By contrast, HECO-PDE got the lowest rank value, with 67 and PMODE also got a lower rank value than CMODE, with 218 . As shown in Table 4, in terms of median solutions with 10D, HECO-DE and CMODE got total rank values, with 91 and 207 respectively. By contrast, HECO-PDE got the lowest rank value, with 87 while PMODE got the same rank value with CMODE, with 207.

\begin{tabular}{|c|c|c|c|c|c|c|c|c|c|c|c|c|c|c|c|c|c|c|c|c|c|c|c|c|c|c|c|c|c|}
\hline Problem & 1 & 2 & 3 & 4 & 5 & 6 & 7 & 8 & 9 & 10 & 11 & 12 & 13 & 14 & 15 & 16 & 17 & 18 & 19 & 20 & 21 & 22 & 23 & 24 & 25 & 26 & 27 & 28 & Total \\
\hline CAL_LSAHDE(2017) & 1 & 1 & 10 & 10 & 12 & 6 & 7 & 12 & 11 & 12 & 7 & 1 & 12 & 7 & 8 & 10 & 11 & 12 & 1 & 8 & 12 & 12 & 9 & 10 & 10 & 12 & 10 & 1 & 235 \\
\hline LSHADE44+IDE(2017) & 1 & 1 & 8 & 7 & 1 & 12 & 6 & 1 & 1 & 2 & 1 & 3 & 1 & 9 & 5 & 8 & 6 & 10 & 4 & 4 & 2 & 7 & 8 & 8 & 8 & 6 & 11 & 10 & 151 \\
\hline LSAHDE44(2017) & 1 & 1 & 9 & 5 & 1 & 11 & 5 & 1 & 10 & 2 & 2 & 10 & 1 & 8 & 9 & 9 & 7 & 9 & 2 & 1 & 4 & 9 & 7 & 7 & 9 & 7 & 9 & 11 & 167 \\
\hline $\operatorname{UDE}(2017)$ & 1 & 1 & 7 & 8 & 11 & 7 & 4 & 1 & 8 & 2 & 10 & 1 & 11 & 6 & 6 & 7 & 8 & 8 & 9 & 10 & 8 & 11 & 6 & 4 & 7 & 9 & 8 & 7 & 186 \\
\hline MA_ES(2018) & 1 & 1 & 1 & 9 & 1 & 5 & 2 & 1 & 1 & 2 & 5 & 12 & 7 & 10 & 12 & 1 & 12 & 1 & 12 & 9 & 9 & 8 & 10 & 6 & 1 & 8 & 2 & 9 & 158 \\
\hline IUDE(2018) & 1 & 1 & 5 & 3 & 1 & 1 & 8 & 1 & 1 & 2 & 6 & 7 & 1 & 3 & 4 & 1 & 5 & 7 & 4 & 7 & 3 & 10 & 1 & 4 & 1 & 4 & 7 & 6 & 105 \\
\hline LSAHDE_IEpsilon(2018) & 1 & 1 & 6 & 4 & 1 & 8 & 3 & 1 & 1 & 2 & 3 & 5 & 1 & 2 & 7 & 1 & 4 & 2 & 3 & 6 & 5 & 1 & 3 & 9 & 1 & 5 & 1 & 12 & 99 \\
\hline DeCODE & 1 & 1 & 1 & 6 & 1 & 1 & 1 & 11 & 8 & 1 & 9 & 4 & 10 & 1 & 1 & 6 & 3 & 11 & 10 & 5 & 1 & 1 & 5 & 1 & 6 & 3 & 12 & 4 & 125 \\
\hline CMODE & 1 & 1 & 11 & 11 & 1 & 10 & 12 & 1 & 12 & 10 & 12 & 6 & 7 & 11 & 11 & 11 & 9 & 6 & 11 & 12 & 11 & 1 & 11 & 11 & 11 & 11 & 6 & 8 & 236 \\
\hline PMODE & 1 & 1 & 12 & 12 & 1 & 9 & 10 & 1 & 1 & 10 & 11 & 11 & 7 & 12 & 10 & 12 & 10 & 5 & 4 & 11 & 10 & 1 & 12 & 12 & 12 & 10 & 5 & 5 & 218 \\
\hline HECO-DE & 1 & 1 & 1 & 1 & 1 & 1 & 11 & 1 & 1 & 2 & 8 & 9 & 1 & 5 & 2 & 1 & 1 & 4 & 4 & 3 & 7 & 1 & 4 & 3 & 1 & 1 & 3 & 3 & 82 \\
\hline HECO-PDE & 1 & 1 & 1 & 1 & 1 & 1 & 9 & 1 & 1 & 2 & 4 & 8 & 1 & 3 & 2 & 1 & 1 & 3 & 4 & 2 & 6 & 1 & 2 & 2 & 1 & 2 & 4 & 2 & 68 \\
\hline
\end{tabular}

Regarding the test functions with $30 D$, rank values based on mean values and median solution on the 28 test functions are reported in Table 5 and 6 , respectively. As shown in Table 5 , in terms of mean values with 30D, HECO-DE and CMODE got total rank values, with 82 and 307 respectively. By contrast, HECO-PDE got the lowest rank value, with 68 and PMODE got a slightly lower rank value than CMODE, with 305 . As 


\begin{tabular}{|c|c|c|c|c|c|c|c|c|c|c|c|c|c|c|c|c|c|c|c|c|c|c|c|c|c|c|c|c|c|}
\hline Problem & 1 & 2 & 3 & 4 & 5 & 6 & 7 & 8 & 9 & 10 & 11 & 12 & 13 & 14 & 15 & 16 & 17 & 18 & 19 & 20 & 21 & 22 & 23 & 24 & 25 & 26 & 27 & 28 & Total \\
\hline CAL_LSAHDE(2017) & 1 & 1 & 10 & 10 & 1 & 8 & 5 & 12 & 12 & 1 & 4 & 3 & 1 & 8 & 8 & 10 & 10 & 11 & 1 & 8 & 4 & 1 & 9 & 12 & 10 & 10 & 11 & 1 & 183 \\
\hline LSHADE44+IDE(2017) & 1 & 1 & 9 & 5 & 1 & 10 & 6 & 1 & 1 & 2 & 2 & 4 & 1 & 10 & 7 & 9 & 6 & 10 & 4 & 4 & 5 & 1 & 10 & 9 & 9 & 8 & 10 & 2 & 148 \\
\hline LSAHDE44(2017) & 1 & 1 & 8 & 7 & 1 & 9 & 7 & 1 & 1 & 2 & 4 & 12 & 1 & 9 & 10 & 8 & 7 & 8 & 2 & 1 & 1 & 1 & 8 & 8 & 8 & 6 & 9 & 11 & 152 \\
\hline UDE(2017) & 1 & 1 & 6 & 8 & 1 & 7 & 4 & 1 & 10 & 2 & 10 & 1 & 1 & 1 & 5 & 7 & 9 & 9 & 11 & 10 & 1 & 1 & 1 & 5 & 7 & 9 & 8 & 7 & 144 \\
\hline MA_ES(2018) & 1 & 1 & 1 & 9 & 1 & 1 & 2 & 1 & 1 & 2 & 2 & 5 & 1 & 3 & 12 & 1 & 8 & 1 & 10 & 9 & 6 & 1 & 3 & 7 & 1 & 7 & 1 & 10 & 108 \\
\hline $\operatorname{IUDE}(2018)$ & 1 & 1 & 1 & 1 & 1 & 1 & 8 & 1 & 1 & 2 & 1 & 8 & 1 & 3 & 5 & 1 & 1 & 6 & 4 & 7 & 7 & 1 & 4 & 5 & 1 & 1 & 7 & 7 & 88 \\
\hline LSAHDE_IEpsilon(2018) & 1 & 1 & 7 & 4 & 1 & 1 & 3 & 1 & 1 & 2 & 6 & 6 & 1 & 3 & 4 & 1 & 5 & 2 & 3 & 6 & 11 & 1 & 6 & 3 & 1 & 5 & 2 & 12 & 100 \\
\hline DeCODE & 1 & 1 & 1 & 6 & 1 & 1 & 1 & 1 & 10 & 2 & 9 & 1 & 1 & 1 & 1 & 6 & 4 & 12 & 12 & 5 & 1 & 1 & 1 & 1 & 6 & 4 & 12 & 9 & 112 \\
\hline CMODE & 1 & 1 & 11 & 12 & 1 & 11 & 11 & 1 & 1 & 12 & 12 & 7 & 1 & 11 & 11 & 11 & 11 & 5 & 4 & 12 & 8 & 1 & 11 & 10 & 11 & 11 & 6 & 2 & 207 \\
\hline PMODE & 1 & 1 & 12 & 11 & 1 & 12 & 12 & 1 & 1 & 2 & 11 & 9 & 1 & 12 & 9 & 12 & 12 & 7 & 4 & 11 & 10 & 1 & 12 & 11 & 12 & 12 & 5 & 2 & 207 \\
\hline HECO-DE & 1 & 1 & 1 & 1 & 1 & 1 & 9 & 1 & 1 & 2 & 8 & 11 & 1 & 6 & 2 & 1 & 2 & 4 & 4 & 3 & 9 & 1 & 7 & 4 & 1 & 3 & 3 & 2 & 91 \\
\hline HECO-PDE & 1 & 1 & 1 & 1 & 1 & 1 & 10 & 1 & 1 & 2 & 7 & 10 & 1 & 6 & 2 & 1 & 2 & 3 & 4 & 2 & 12 & 1 & 5 & 2 & 1 & 2 & 4 & 2 & 87 \\
\hline
\end{tabular}

shown in Table 6, in terms of median solutions with 30D, HECO-DE and CMODE got total rank values, with 83 and 311 respectively. By contrast, HECO-PDE got the lowest rank value, with 71 and PMODE got lower rank value than CMODE, with 306.

\begin{tabular}{|c|c|c|c|c|c|c|c|c|c|c|c|c|c|c|c|c|c|c|c|c|c|c|c|c|c|c|c|c|c|}
\hline Problem & 1 & 2 & 3 & 4 & 5 & 6 & 7 & 8 & 9 & 10 & 11 & 12 & 13 & 14 & 15 & 16 & 17 & 18 & 19 & 20 & 21 & 22 & 23 & 24 & 25 & 26 & 27 & 28 & Total \\
\hline CAL_LSAHDE(2017) & 1 & 1 & 10 & 10 & 10 & 6 & 8 & 1 & 10 & 10 & 4 & 10 & 10 & 10 & 11 & 12 & 10 & 11 & 1 & 3 & 10 & 10 & 10 & 12 & 10 & 10 & 10 & 1 & 222 \\
\hline LSHADE44+IDE(2017) & 1 & 1 & 9 & 6 & 1 & 11 & 4 & 10 & 1 & 9 & 3 & 7 & 7 & 9 & 7 & 8 & 7 & 9 & 4 & 5 & 8 & 7 & 9 & 9 & 8 & 7 & 11 & 9 & 187 \\
\hline LSAHDE44(2017) & 1 & 1 & 8 & 4 & 1 & 10 & 5 & 2 & 1 & 1 & 2 & 5 & 6 & 8 & 8 & 9 & 6 & 6 & 2 & 1 & 7 & 8 & 7 & 8 & 9 & 6 & 9 & 10 & 151 \\
\hline $\operatorname{UDE}(2017)$ & 1 & 1 & 5 & 9 & 7 & 4 & 2 & 9 & 7 & 8 & 8 & 8 & 8 & 6 & 6 & 7 & 8 & 7 & 9 & 9 & 4 & 6 & 5 & 6 & 6 & 8 & 8 & 6 & 178 \\
\hline MA_ES(2018) & 1 & 1 & 1 & 8 & 1 & 3 & 1 & 2 & 1 & 1 & 1 & 9 & 1 & 7 & 12 & 1 & 9 & 2 & 10 & 10 & 9 & 1 & 6 & 7 & 1 & 9 & 1 & 4 & 120 \\
\hline IUDE(2018) & 1 & 1 & 6 & 5 & 1 & 7 & 7 & 2 & 7 & 1 & 5 & 2 & 5 & 1 & 5 & 4 & 3 & 5 & 7 & 8 & 6 & 5 & 4 & 3 & 5 & 2 & 5 & 7 & 120 \\
\hline LSAHDE_IEpsilon(2018) & 1 & 1 & 7 & 1 & 1 & 9 & 3 & 2 & 1 & 1 & 9 & 6 & 9 & 5 & 3 & 6 & 5 & 1 & 3 & 2 & 3 & 9 & 2 & 5 & 7 & 5 & 2 & 12 & 121 \\
\hline DeCODE & 1 & 1 & 1 & 7 & 9 & 5 & 6 & 8 & 9 & 1 & 12 & 1 & 1 & 2 & 4 & 5 & 4 & 12 & 8 & 4 & 5 & 4 & 8 & 4 & 4 & 1 & 12 & 5 & 144 \\
\hline CMODE & 12 & 12 & 12 & 11 & 12 & 8 & 11 & 12 & 12 & 12 & 10 & 12 & 11 & 11 & 10 & 10 & 12 & 10 & 11 & 11 & 12 & 12 & 11 & 10 & 11 & 11 & 7 & 11 & 307 \\
\hline PMODE & 11 & 11 & 11 & 12 & 11 & 12 & 12 & 11 & 11 & 11 & 11 & 11 & 12 & 12 & 9 & 11 & 11 & 8 & 12 & 12 & 11 & 11 & 12 & 11 & 12 & 12 & 6 & 8 & 305 \\
\hline HECO-DE & 1 & 1 & 1 & 2 & 8 & 1 & 9 & 2 & 1 & 1 & 7 & 4 & 1 & 4 & 1 & 1 & 1 & 4 & 4 & 6 & 2 & 3 & 3 & 2 & 1 & 4 & 3 & 3 & 81 \\
\hline HECO-PDE & 1 & 1 & 1 & 2 & 1 & 1 & 10 & 2 & 1 & 1 & 6 & 3 & 1 & 3 & 1 & 1 & 2 & 3 & 4 & 7 & 1 & 2 & 1 & 1 & 1 & 3 & 4 & 2 & 67 \\
\hline
\end{tabular}

\begin{tabular}{|c|c|c|c|c|c|c|c|c|c|c|c|c|c|c|c|c|c|c|c|c|c|c|c|c|c|c|c|c|c|}
\hline Problem & 1 & 2 & 3 & 4 & 5 & 6 & 7 & 8 & 9 & 10 & 11 & 12 & 13 & 14 & 15 & 16 & 17 & 18 & 19 & 20 & 21 & 22 & 23 & 24 & 25 & 26 & 27 & 28 & Total \\
\hline CAL_LSAHDE(2017) & 1 & 1 & 10 & 10 & 1 & 6 & 8 & 1 & 1 & 1 & 4 & 8 & 10 & 7 & 9 & 12 & 10 & 9 & 1 & 3 & 3 & 10 & 6 & 12 & 12 & 10 & 9 & 1 & 176 \\
\hline LSHADE44+IDE(2017) & 1 & 1 & 9 & 2 & 1 & 10 & 6 & 10 & 2 & 10 & 3 & 2 & 1 & 10 & 7 & 8 & 8 & 11 & 4 & 5 & 9 & 7 & 9 & 9 & 8 & 5 & 11 & 9 & 178 \\
\hline LSAHDE44(2017) & 1 & 1 & 8 & 5 & 1 & 9 & 5 & 2 & 2 & 2 & 2 & 7 & 8 & 9 & 8 & 9 & 7 & 5 & 2 & 1 & 8 & 8 & 8 & 7 & 9 & 8 & 10 & 10 & 162 \\
\hline $\operatorname{UDE}(2017)$ & 1 & 1 & 5 & 9 & 1 & 4 & 2 & 9 & 8 & 9 & 8 & 9 & 7 & 6 & 6 & 5 & 9 & 7 & 7 & 9 & 3 & 6 & 1 & 6 & 6 & 9 & 7 & 6 & 166 \\
\hline MA_ES(2018) & 1 & 1 & 1 & 8 & 1 & 3 & 1 & 2 & 2 & 2 & 1 & 10 & 1 & 8 & 12 & 1 & 6 & 1 & 10 & 10 & 10 & 1 & 7 & 8 & 1 & 6 & 1 & 4 & 120 \\
\hline IUDE & 1 & 1 & 6 & 6 & 1 & 8 & 4 & 2 & 8 & 2 & 6 & 4 & 1 & 1 & 3 & 4 & 3 & 6 & 7 & 8 & 3 & 1 & 1 & 1 & 5 & 1 & 5 & 7 & 106 \\
\hline LSAHDE_IEpsilon(2018) & 1 & 1 & 7 & 1 & 1 & 7 & 3 & 2 & 2 & 2 & 9 & 5 & 9 & 3 & 5 & 7 & 5 & 2 & 3 & 2 & 7 & 9 & 4 & 5 & 7 & 2 & 2 & 12 & 125 \\
\hline DeCODE & 1 & 1 & 1 & 6 & 1 & 5 & 7 & 2 & 8 & 2 & 12 & 1 & 1 & 1 & 3 & 5 & 4 & 12 & 9 & 4 & 3 & 1 & 10 & 4 & 4 & 7 & 12 & 5 & 132 \\
\hline CMODE & 12 & 11 & 12 & 11 & 12 & 11 & 11 & 12 & 12 & 11 & 11 & 12 & 11 & 11 & 11 & 10 & 12 & 10 & 11 & 11 & 12 & 12 & 11 & 10 & 11 & 11 & 8 & 11 & 311 \\
\hline PMODE & 11 & 12 & 11 & 12 & 11 & 12 & 12 & 11 & 11 & 12 & 10 & 11 & 12 & 12 & 10 & 11 & 11 & 8 & 12 & 12 & 11 & 11 & 12 & 11 & 10 & 12 & 6 & 8 & 305 \\
\hline HECO-DE & 1 & 1 & 1 & 2 & 1 & 1 & 9 & 2 & 2 & 2 & 7 & 6 & 1 & 4 & 1 & 1 & 2 & 4 & 4 & 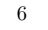 & 2 & 5 & 5 & 3 & 1 & 4 & 3 & 2 & 83 \\
\hline HECO-PDE & 1 & 1 & 1 & 2 & 1 & 1 & 10 & 2 & 2 & 2 & 5 & 3 & 1 & 4 & 1 & 1 & 1 & 3 & 4 & 7 & 1 & 1 & 3 & 2 & 1 & 3 & 4 & 3 & 71 \\
\hline
\end{tabular}

Regarding the test functions with $50 D$, rank values based on mean values and median solution on the 28 test functions are reported in Table 7 and 8, respectively. As shown in Table 7, in terms of mean values with 50D, HECO-DE and CMODE got total rank values, with 90 and 314 respectively. By contrast, HECO-PDE got the lowest rank value, with 77 and PMODE got a slightly lower rank value than CMODE, with 312 . As shown in Table 8, in terms of median solutions with 50D, HECO-DE and CMODE got total rank values, with 87 and 314 respectively. By contrast, HECO-PDE got the lowest rank value, with 75 while PMODE got a higher rank value than CMODE, with 318.

Regarding the test functions with $100 D$, rank values based on mean values and median solution on the 28 test functions are reported in Table 9 and 10, respectively. As shown in Table 9, in terms of mean values with 100D, HECO-DE got highest total rank value with 87 and CMODE got 315 respectively. By contrast, HECO-PDE got higher rank value than HECO-DE with 88 while PMODE got a lower rank value than CMODE, with 305. As shown in Table 10, in terms of median solutions with 100D, HECO-DE and CMODE got total rank values, with 96 and 315 respectively. By contrast, HECO-PDE got the lowest rank value with 89, while PMODE got a higher rank value than CMODE with 321.

According to the CEC 2018 competition rules, the ranks of HECO-PDE and HECO-DE are on the top two on each dimension but CMODE and PMODE on the bottom two. This result confirms our guess that MOEAs 
Table 7: Ranks based on mean values on the 28 functions of $\mathbf{5 0}$ dimensions

\begin{tabular}{|c|c|c|c|c|c|c|c|c|c|c|c|c|c|c|c|c|c|c|c|c|c|c|c|c|c|c|c|c|c|}
\hline & & & & & & & & & & & & & & & & & & & & & & & & & & & & & \\
\hline Problem & 1 & 2 & 3 & 4 & 5 & 6 & 7 & 8 & 9 & 10 & 11 & 12 & 13 & 14 & 15 & 16 & 17 & 18 & 19 & 20 & 21 & 22 & 23 & 24 & 25 & 26 & 27 & 28 & Total \\
\hline CAL_LSAHDE(2017) & 10 & 10 & 10 & 10 & 9 & 4 & 8 & 10 & 10 & 8 & 4 & 7 & 10 & 10 & 10 & 10 & 10 & 8 & 1 & 12 & 6 & 9 & 10 & 10 & 10 & 10 & 7 & 1 & 234 \\
\hline LSHADE44+IDE(2017) & 9 & 1 & 9 & 5 & 1 & 10 & 5 & 9 & 7 & 10 & 1 & 4 & 7 & 9 & 7 & 8 & 8 & 7 & 4 & 4 & 9 & 5 & 8 & 9 & 9 & 8 & 10 & 9 & 192 \\
\hline LSAHDE44(2017) & 1 & 1 & 8 & 3 & 1 & 9 & 4 & 1 & 3 & 1 & 2 & 9 & 6 & 8 & 8 & 9 & 7 & 6 & 3 & 1 & 10 & 6 & 6 & 8 & 8 & 7 & 6 & 8 & 150 \\
\hline $\mathrm{UDE}(2017)$ & 1 & 1 & 5 & 9 & 10 & 3 & 2 & 8 & 1 & 9 & 5 & 6 & 9 & 6 & 6 & 6 & 9 & 11 & 9 & 7 & 3 & 8 & 5 & 6 & 6 & 9 & 11 & 5 & 176 \\
\hline MA_ES(2018) & 1 & 1 & 1 & 8 & 1 & 1 & 1 & 2 & 8 & 1 & 3 & 10 & 8 & 7 & 9 & 1 & 6 & 1 & 10 & 9 & 8 & 4 & 7 & 7 & 1 & 5 & 1 & 4 & 126 \\
\hline IUDE(2018) & 1 & 1 & 6 & 7 & 1 & 11 & 7 & 4 & 1 & 1 & 7 & 3 & 5 & 1 & 3 & 5 & 3 & 5 & 8 & 8 & 4 & 3 & 4 & 3 & 5 & 1 & 5 & 7 & 120 \\
\hline LSAHDE_IEpsilon(2018) & 1 & 1 & 7 & 4 & 8 & 8 & 3 & 7 & 6 & 7 & 8 & 8 & 4 & 4 & 5 & 7 & 5 & 2 & 7 & 2 & 5 & 10 & 2 & 5 & 7 & 6 & 2 & 10 & 151 \\
\hline DeCODE & 1 & 1 & 1 & 6 & 1 & 2 & 6 & 3 & 9 & 6 & 12 & 5 & 1 & 2 & 4 & 4 & 4 & 12 & 2 & 3 & 7 & 2 & 9 & 4 & 4 & 4 & 12 & 6 & 133 \\
\hline CMODE & 12 & 12 & 11 & 11 & 11 & 12 & 12 & 12 & 11 & 12 & 11 & 11 & 12 & 12 & 12 & 11 & 12 & 9 & 12 & 10 & 11 & 11 & 11 & 11 & 11 & 11 & 9 & 11 & 314 \\
\hline PMODE & 11 & 11 & 12 & 12 & 12 & 7 & 11 & 11 & 12 & 11 & 10 & 12 & 11 & 11 & 11 & 12 & 11 & 10 & 11 & 11 & 12 & 12 & 12 & 12 & 12 & 12 & 8 & 12 & 312 \\
\hline HECO-DE & 1 & 1 & 1 & 1 & 1 & 6 & 9 & 6 & 3 & 5 & 9 & 1 & 3 & 5 & 1 & 1 & 2 & 3 & 4 & 5 & 1 & 7 & 3 & 2 & 1 & 3 & 3 & 2 & 90 \\
\hline HECO-PDE & 1 & 1 & 1 & 1 & 1 & 5 & 10 & 5 & 3 & 4 & 6 & 2 & 2 & 3 & 1 & 1 & 1 & 4 & 4 & 6 & 2 & 1 & 1 & 1 & 1 & 2 & 4 & 3 & 77 \\
\hline
\end{tabular}

\begin{tabular}{|c|c|c|c|c|c|c|c|c|c|c|c|c|c|c|c|c|c|c|c|c|c|c|c|c|c|c|c|c|c|}
\hline Problem & 1 & 2 & 3 & 4 & 5 & 6 & 7 & 8 & 9 & 10 & 11 & 12 & 13 & 14 & 15 & 16 & 17 & 18 & 0 & 20 & 21 & 22 & 23 & 2 & 5 & 2 & 87 & 28 & \\
\hline CAL_LSAHDE(2017) & 1 & 1 & 10 & 10 & 1 & 6 & 8 & 10 & 7 & 8 & 4 & 7 & 10 & 6 & 12 & 10 & 10 & 9 & 1 & 3 & 7 & 9 & 6 & 10 & 10 & 10 & 7 & 10 & Iotal \\
\hline LSHADE44+IDE(2017) & 1 & 1 & 9 & 2 & 1 & 9 & 5 & 9 & 9 & 10 & 3 & 3 & 8 & 10 & 7 & 9 & 8 & 8 & 5 & 5 & 9 & 7 & 9 & 9 & 9 & 8 & 11 & 9 & 193 \\
\hline LSAHDE44(2017) & 1 & 1 & 8 & 5 & 1 & 8 & 4 & 1 & 3 & 1 & 1 & 10 & 7 & 9 & 9 & 8 & 7 & 6 & 4 & 1 & 10 & 8 & 8 & 8 & 8 & 7 & 6 & 8 & 158 \\
\hline $\mathrm{UDE}(2017)$ & 1 & 1 & 5 & 9 & 10 & 5 & 2 & 8 & 1 & 9 & 5 & 5 & 9 & 7 & 6 & 6 & 9 & 7 & 10 & 8 & 0 & 6 & 2 & 7 & 6 & 9 & 10 & 5 & 169 \\
\hline MA_ES(2018) & 1 & 1 & 1 & 8 & 1 & 3 & 1 & 2 & 8 & 1 & 2 & 9 & 1 & 8 & 8 & 1 & 5 & 1 & 9 & 10 & 8 & 5 & 7 & 6 & 1 & 4 & 1 & 4 & 117 \\
\hline IUDE(2018) & 1 & 1 & 6 & 7 & 1 & 10 & 7 & 3 & 1 & 1 & 6 & 4 & 1 & 3 & 1 & 5 & 1 & 5 & 2 & 9 & 4 & 4 & 2 & 1 & 4 & 2 & 5 & 7 & 104 \\
\hline LSAHDE_IEpsilon(2018) & 1 & 1 & 7 & 1 & 1 & 7 & 3 & 7 & 3 & 7 & 8 & 8 & 6 & 1 & 5 & 7 & 6 & 2 & 8 & 2 & 5 & 10 & 5 & 5 & 7 & 6 & 2 & 10 & 141 \\
\hline DeCODE & 1 & 1 & 1 & 6 & 1 & 4 & 6 & 6 & 10 & 6 & 12 & 5 & 1 & 3 & 4 & 4 & 4 & 12 & 3 & 4 & 6 & 3 & 10 & 4 & 4 & 5 & 12 & 6 & 144 \\
\hline CMODE & 12 & 12 & 12 & 11 & 11 & 12 & 12 & 12 & 11 & 11 & 11 & 12 & 12 & 11 & 10 & 11 & 11 & 10 & 12 & 11 & 11 & 11 & 12 & 11 & 11 & 11 & 8 & 12 & 314 \\
\hline PMODE & 11 & 11 & 11 & 12 & 12 & 11 & 11 & 11 & 12 & 12 & 10 & 11 & 11 & 12 & 11 & 12 & 12 & 11 & 11 & 12 & 12 & 12 & 11 & 12 & 12 & 12 & 9 & 11 & 318 \\
\hline HECO-DE & 1 & 1 & 1 & 2 & 1 & 2 & 9 & 4 & 3 & 4 & 9 & 2 & 1 & 5 & 2 & 1 & 3 & 4 & 5 & 6 & 3 & 2 & 4 & 3 & 1 & 3 & 3 & 2 & 87 \\
\hline HECO-PDE & 1 & 1 & 1 & 2 & 1 & 1 & 10 & 5 & 3 & 4 & 7 & 1 & 1 & 2 & 2 & 1 & 2 & 3 & 5 & 7 & 2 & 1 & 1 & 2 & 1 & 1 & 4 & 3 & 75 \\
\hline
\end{tabular}

based on non-dominance, such as PMODE and CMODE, may not perform as good as decomposition-based MOEAs, such as HECO-PDE and HECO-DE for solving COPs.

Table 9: Ranks based on mean values on the 28 functions of $\mathbf{1 0 0}$ dimensions

\begin{tabular}{|c|c|c|c|c|c|c|c|c|c|c|c|c|c|c|c|c|c|c|c|c|c|c|c|c|c|c|c|c|c|}
\hline Problem & 1 & 2 & 3 & 4 & 5 & 6 & 7 & 8 & 9 & 10 & 11 & 12 & 13 & 14 & 15 & 16 & 17 & 18 & 19 & 20 & 21 & 22 & 23 & 24 & 25 & 26 & 27 & 28 & Total \\
\hline CAL_LSAHDE(2017) & 9 & 9 & 11 & 10 & 6 & 3 & 9 & 10 & 8 & 9 & 5 & 5 & 9 & 10 & 10 & 10 & 10 & 11 & 1 & 12 & 10 & 7 & 10 & 10 & 10 & 10 & 9 & 1 & 234 \\
\hline LSHADE44+IDE(2017) & 10 & 10 & 8 & 4 & 4 & 11 & 4 & 6 & 2 & 7 & 1 & 1 & 6 & 9 & 5 & 8 & 8 & 7 & 3 & 4 & 5 & 6 & 6 & 7 & 9 & 8 & 10 & 7 & 176 \\
\hline LSAHDE44(2017) & 1 & 1 & 7 & 3 & 3 & 10 & 5 & 1 & 1 & 1 & 4 & 10 & 7 & 8 & 8 & 9 & 6 & 6 & 2 & 3 & 9 & 5 & 8 & 6 & 8 & 6 & 6 & 9 & 153 \\
\hline $\operatorname{UDE}(2017)$ & 8 & 8 & 5 & 9 & 10 & 4 & 2 & 9 & 10 & 8 & 6 & 2 & 10 & 5 & 7 & 5 & 9 & 8 & 9 & 8 & 4 & 10 & 2 & 8 & 5 & 9 & 11 & 5 & 196 \\
\hline MA_ES(2018) & 1 & 1 & 1 & 7 & 9 & 1 & 1 & 2 & 9 & 4 & 2 & 8 & 4 & 7 & 9 & 1 & 5 & 1 & 10 & 9 & 8 & 2 & 7 & 4 & 1 & 5 & 3 & 4 & 126 \\
\hline IUDE(2018) & 1 & 1 & 9 & 8 & 5 & 12 & 7 & 3 & 6 & 5 & 3 & 3 & 8 & 1 & 4 & 6 & 3 & 5 & 8 & 7 & 2 & 8 & 5 & 5 & 6 & 3 & 5 & 8 & 147 \\
\hline LSAHDE_IEpsilon(2018) & 7 & 7 & 6 & 5 & 8 & 9 & 3 & 7 & 7 & 6 & 8 & 4 & 5 & 4 & 6 & 7 & 7 & 2 & 6 & 2 & 3 & 9 & 4 & 9 & 7 & 7 & 4 & 10 & 169 \\
\hline DeCODE & 1 & 1 & 1 & 6 & 7 & 2 & 6 & 8 & 3 & 10 & 10 & 6 & 3 & 6 & 3 & 4 & 4 & 12 & 7 & 1 & 1 & 1 & 9 & 3 & 4 & 4 & 12 & 6 & 141 \\
\hline CMODE & 12 & 12 & 10 & 12 & 12 & 8 & 12 & 11 & 12 & 12 & 11 & 11 & 12 & 11 & 12 & 12 & 11 & 10 & 12 & 11 & 12 & 12 & 11 & 11 & 12 & 12 & 8 & 11 & 315 \\
\hline PMODE & 11 & 11 & 12 & 11 & 11 & 6 & 11 & 12 & 11 & 11 & 12 & 12 & 11 & 12 & 11 & 11 & 12 & 9 & 11 & 10 & 11 & 11 & 12 & 12 & 11 & 11 & 7 & 12 & 305 \\
\hline HECO-DE & 1 & 1 & 1 & 1 & 1 & 7 & 8 & 4 & 3 & 2 & 9 & 7 & 1 & 3 & 1 & 1 & 1 & 3 & 3 & 5 & 7 & 3 & 3 & 2 & 3 & 2 & 2 & 2 & 87 \\
\hline HECO-PDE & 1 & 1 & 1 & 2 & 1 & 5 & 10 & 5 & 3 & 3 & 7 & 9 & 2 & 2 & 1 & 1 & 2 & 4 & 3 & 6 & 6 & 4 & 1 & 1 & 2 & 1 & 1 & 3 & 88 \\
\hline
\end{tabular}

\section{Conclusions}

From an experimental observation, we find that given a valley landscape, the maximal variance direction in a population can be regarded as the valley direction. Based on this finding, a new search operator, called PCA-projection, is proposed, in which PCA is used to project points along the maximal variance direction. PCA-projection can be easily added into an existing MOEA through a mixed strategy. We design two MOEAs enhanced with PCA-projection, called HECO-PDE and PMODE, for evaluating the effectiveness of this new operator. Experimental results show that an EA enhanced with PCA-projection performs better than its corresponding opponent without this operator. Furthermore, HECO-PDE is ranked first on all dimensions when compared with the state-of-art single-objective EAs from the IEEE CEC 2018 competition and another recent MOEA (DeCODE) for constrained optimisation. This study also reveals that decomposition-based MOEAs, such as HECO-PDE and HECO-DE, are competitive with best singleobjective and multi-objective EAs in constrained optimisation, but MOEAs based on non-dominance, such as PMODE and CMODE, may not perform so well. For the future work, PCA-projection can be applied to other EAs. 


\begin{tabular}{|c|c|c|c|c|c|c|c|c|c|c|c|c|c|c|c|c|c|c|c|c|c|c|c|c|c|c|c|c|c|}
\hline Problem & 1 & 2 & 3 & 4 & 5 & 6 & 7 & 8 & 9 & 10 & 11 & 12 & 13 & 14 & 15 & 16 & 17 & 18 & 19 & 20 & 21 & 22 & 23 & 24 & 25 & 26 & 27 & 28 & Total \\
\hline CAL_LSAHDE(2017) & 9 & 9 & 10 & 10 & 7 & 5 & 9 & 10 & 6 & 9 & 5 & 4 & 9 & 1 & 10 & 10 & 10 & 9 & 1 & 1 & 3 & 7 & 6 & 10 & 10 & 10 & 10 & 1 & 201 \\
\hline LSHADE44+IDE(2017) & 10 & 10 & 9 & 1 & 3 & 9 & 5 & 6 & 2 & 8 & 4 & 2 & 6 & 10 & 6 & 8 & 7 & 6 & 3 & 5 & 7 & 5 & 7 & 8 & 9 & 7 & 6 & 8 & 177 \\
\hline LSAHDE44(2017) & 1 & 1 & 8 & 4 & 4 & 8 & 6 & 1 & 1 & 1 & 2 & 10 & 7 & 9 & 9 & 9 & 5 & 7 & 2 & 4 & 8 & 4 & 9 & 9 & 8 & 4 & 7 & 9 & 157 \\
\hline UDE(2017) & 8 & 8 & 5 & 9 & 10 & 4 & 2 & 9 & 8 & 10 & 6 & 1 & 10 & 6 & 8 & 5 & 9 & 8 & 9 & 9 & 5 & 10 & 3 & 6 & 5 & 8 & 8 & 5 & 194 \\
\hline MA_ES(2018) & 1 & 1 & 1 & 7 & 9 & 2 & 1 & 2 & 10 & 5 & 1 & 6 & 4 & 8 & 4 & 1 & 4 & 1 & 10 & 10 & 6 & 3 & 8 & 4 & 1 & 5 & 1 & 4 & 120 \\
\hline IUDE(2018) & 1 & 1 & 7 & 8 & 5 & 10 & 4 & 3 & 9 & 4 & 3 & 3 & 8 & 2 & 7 & 6 & 3 & 5 & 7 & 8 & 2 & 8 & 5 & 5 & 6 & 3 & 5 & 7 & 145 \\
\hline LSAHDE_IEpsilon(2018) & 7 & 7 & 6 & 5 & 8 & 6 & 3 & 7 & 7 & 6 & 8 & 5 & 5 & 5 & 4 & 7 & 6 & 2 & 6 & 3 & 4 & 9 & 4 & 7 & 7 & 6 & 4 & 10 & 164 \\
\hline DeCODE & 1 & 1 & 1 & 6 & 6 & 3 & 7 & 8 & 3 & 7 & 12 & 7 & 3 & 7 & 3 & 4 & 8 & 12 & 8 & 2 & 1 & 1 & 10 & 3 & 4 & 9 & 12 & 6 & 155 \\
\hline CMODE & 12 & 12 & 11 & 12 & 11 & 11 & 12 & 11 & 12 & 12 & 10 & 11 & 11 & 11 & 11 & 12 & 11 & 10 & 11 & 11 & 12 & 12 & 11 & 11 & 11 & 12 & 11 & 11 & 316 \\
\hline PMODE & 11 & 11 & 12 & 11 & 12 & 12 & 11 & 12 & 11 & 11 & 11 & 12 & 12 & 12 & 12 & 11 & 12 & 11 & 12 & 12 & 11 & 11 & 12 & 12 & 12 & 11 & 9 & 12 & 321 \\
\hline HECO-DE & 1 & 1 & 1 & 3 & 1 & 7 & 8 & 4 & 3 & 2 & 9 & 9 & 2 & 4 & 1 & 1 & 1 & 3 & 3 & 6 & 10 & 2 & 2 & 2 & 3 & 2 & 3 & 2 & 96 \\
\hline HECO-PDE & 1 & 1 & 1 & 1 & 1 & 1 & 10 & 5 & 3 & 3 & 7 & 8 & 1 & 3 & 1 & 1 & 2 & 4 & 3 & 7 & 9 & 6 & 1 & 1 & 2 & 1 & 2 & 3 & 89 \\
\hline
\end{tabular}

\section{References}

[1] Z. Michalewicz, M. Schoenauer, Evolutionary algorithms for constrained parameter optimization problems, Evolutionary computation 4 (1) (1996) 1-32.

[2] C. A. Coello Coello, Theoretical and numerical constraint-handling techniques used with evolutionary algorithms: A survey of the state of the art, Computer Methods in Applied Mechanics and Engineering 191 (11-12) (2002) 1245-1287.

[3] E. Mezura-Montes, C. A. Coello Coello, Constraint-handling in nature-inspired numerical optimization: past, present and future, Swarm and Evolutionary Computation 1 (4) (2011) 173-194.

[4] C. Segura, C. A. C. Coello, G. Miranda, C. León, Using multi-objective evolutionary algorithms for single-objective constrained and unconstrained optimization, Annals of Operations Research 240 (1) (2016) 217-250.

[5] P. D. Surry, N. J. Radcliffe, The COMOGA method: constrained optimisation by multi-objective genetic algorithms, Control and Cybernetics 26 (1997) 391-412.

[6] Y. Zhou, Y. Li, J. He, L. Kang, Multi-objective and MGG evolutionary algorithm for constrained optimisation, in: Proceedings of 2003 IEEE Congress on Evolutionary Computation, IEEE Press, Canberra, Australia, 2003 , pp. 1-5.

[7] J. He, T. Chen, X. Yao, On the easiest and hardest fitness functions, IEEE Transactions on Evolutionary Computation 19 (2) (2015) 295-305.

[8] C. Munteanu, V. Lazarescu, Improving mutation capabilities in a real-coded genetic algorithm, in: Workshops on Applications of Evolutionary Computation, Springer, 1999, pp. 138-149.

[9] Y.-l. Li, J. Zhang, W.-n. Chen, Differential evolution algorithm with pca-based crossover, in: Proceedings of the 14th annual conference companion on Genetic and evolutionary computation, ACM, 2012, pp. 1509-1510.

[10] W. Chu, X. Gao, S. Sorooshian, Fortify particle swam optimizer (pso) with principal components analysis: A case study in improving bound-handling for optimizing high-dimensional and complex problems, in: 2011 IEEE Congress of Evolutionary Computation (CEC), IEEE, 2011, pp. 1644-1648.

[11] X. Zhao, W. Lin, Q. Zhang, Enhanced particle swarm optimization based on principal component analysis and line search, Applied Mathematics and Computation 229 (2014) 440-456.

[12] B. T. Ong, M. Fukushima, Automatically terminated particle swarm optimization with principal component analysis, International Journal of Information Technology \& Decision Making 14 (01) (2015) 171-194.

[13] G. Xu, X. Zhao, R. Li, Cooperative co-evolution with principal component analysis for large scale optimization, in: International Conference on Bio-Inspired Computing: Theories and Applications, Springer, 2018 , pp. $426-434$.

[14] Z. Cui, F. Li, W. Zhang, Bat algorithm with principal component analysis, International Journal of Machine Learning and Cybernetics (2018) 1-20.

[15] Q. Zhang, A. Zhou, Y. Jin, RM-MEDA: A regularity model-based multiobjective estimation of distribution algorithm, IEEE Transactions on Evolutionary Computation 12 (1) (2008) 41-63.

[16] Y. Wang, J. Xiang, Z. Cai, A regularity model-based multiobjective estimation of distribution algorithm with reducing redundant cluster operator, Applied Soft Computing 12 (11) (2012) 3526-3538.

[17] P. N. Suganthan, The CEC 2017 competition on constrained real-parameter optimization (2017). URL http://web.mysites.ntu.edu.sg/epnsugan/PublicSite/SharedDocuments/CEC-2017

[18] P. N. Suganthan, The CEC 2018 competition on constrained real-parameter optimization (2018). URL http://web.mysites.ntu.edu.sg/epnsugan/PublicSite/SharedDocuments/CEC-2018

[19] C. A. Coello Coello, Constraint-handling using an evolutionary multiobjective optimization technique, Civil Engineering Systems 17 (4) (2000) 319-346.

[20] S. Venkatraman, G. G. Yen, A generic framework for constrained optimization using genetic algorithms, IEEE Transactions on Evolutionary Computation 9 (4) (2005) 424-435.

[21] Z. Cai, Y. Wang, A multiobjective optimization-based evolutionary algorithm for constrained optimization, IEEE Transactions on Evolutionary Computation 10 (6) (2006) 658-675.

[22] Y. Wang, Z. Cai, Combining multiobjective optimization with differential evolution to solve constrained optimization problems, IEEE Transactions on Evolutionary Computation 16 (1) (2012) 117-134.

[23] Y. Wang, Z. Cai, A dynamic hybrid framework for constrained evolutionary optimization, IEEE Transactions on Systems, Man, and Cybernetics, Part B: Cybernetics 42 (1) (2012) 203-217.

[24] W.-F. Gao, G. G. Yen, S.-Y. Liu, A dual-population differential evolution with coevolution for constrained optimization, IEEE Transactions on Cybernetics 45 (5) (2015) 1094-1107. 
[25] L. Gao, Y. Zhou, X. Li, Q. Pan, W. Yi, Multi-objective optimization based reverse strategy with differential evolution algorithm for constrained optimization problems, Expert Systems with Applications 42 (14) (2015) 5976-5987.

[26] X. Li, S. Zeng, C. Li, J. Ma, Many-objective optimization with dynamic constraint handling for constrained optimization problems, Soft Computing 21 (24) (2017) 7435-7445.

[27] S. Zeng, R. Jiao, C. Li, X. Li, J. S. Alkasassbeh, A general framework of dynamic constrained multiobjective evolutionary algorithms for constrained optimization, IEEE transactions on Cybernetics 47 (9) (2017) 2678-2688.

[28] T. Xu, J. He, C. Shang, W. Ying, A new multi-objective model for constrained optimisation, in: P. Angelov, A. Gegov, C. Jayne, Q. Shen (Eds.), Advances in Computational Intelligence Systems: the 16th UK Workshop on Computational Intelligence, Springer, 2017, pp. 71-85.

[29] B.-C. Wang, H.-X. Li, Q. Zhang, Y. Wang, Decomposition-based multiobjective optimization for constrained evolutionary optimization, IEEE Transactions on Systems, Man, and Cybernetics: Systems.

[30] C. Peng, H.-L. Liu, F. Gu, A novel constraint-handling technique based on dynamic weights for constrained optimization problems, Soft Computing 22 (12) (2018) 3919-3935.

[31] R. Storn, K. Price, Differential evolution-a simple and efficient heuristic for global optimization over continuous spaces, Journal of global optimization 11 (4) (1997) 341-359.

[32] R. Storn, System design by constraint adaptation and differential evolution, IEEE Transactions on Evolutionary Computation 3 (1) (1999) 22-34.

[33] R. Poláková, L-shade with competing strategies applied to constrained optimization, in: Evolutionary Computation (CEC), 2017 IEEE Congress on, IEEE, 2017, pp. 1683-1689.

[34] J. Tvrdık, Competitive differential evolution, in: MENDEL, 2006, pp. 7-12.

[35] J. Tvrdík, Adaptation in differential evolution: A numerical comparison, Applied Soft Computing 9 (3) (2009) $1149-1155$.

[36] J. Zhang, A. C. Sanderson, Jade: adaptive differential evolution with optional external archive, IEEE Transactions on evolutionary computation 13 (5) (2009) 945-958.

[37] P. Kaelo, M. Ali, A numerical study of some modified differential evolution algorithms, European journal of operational research 169 (3) (2006) 1176-1184.

[38] A. Zamuda, Adaptive constraint handling and success history differential evolution for cec 2017 constrained real-parameter optimization, in: Evolutionary Computation (CEC), 2017 IEEE Congress on, IEEE, 2017, pp. 2443-2450.

[39] J. Tvrdík, R. Poláková, A simple framework for constrained problems with application of l-shade44 and ide, in: Evolutionary Computation (CEC), 2017 IEEE Congress on, IEEE, 2017, pp. 1436-1443.

[40] A. Trivedi, K. Sanyal, P. Verma, D. Srinivasan, A unified differential evolution algorithm for constrained optimization problems, in: Evolutionary Computation (CEC), 2017 IEEE Congress on, IEEE, 2017, pp. 1231-1238.

[41] M. Hellwig, H.-G. Beyer, A matrix adaptation evolution strategy for constrained real-parameter optimization, in: 2018 IEEE Congress on Evolutionary Computation (CEC), IEEE, 2018, pp. 1-8.

[42] A. Trived, D. Srinivasan, N. Biswas, An improved unified differential evolution algorithm for constrained optimization problems, in: 2018 IEEE Congress on Evolutionary Computation (CEC), IEEE, 2018, pp. 1-10.

[43] Z. Fan, Y. Fang, W. Li, Y. Yuan, Z. Wang, X. Bian, Lshade44 with an improved $\epsilon$ constraint-handling method for solving constrained single-objective optimization problems, in: 2018 IEEE Congress on Evolutionary Computation (CEC), IEEE, 2018, pp. 1-8. 


\section{Supplement}

This supplement provides detailed experimental results of PMODE and HECO-PDE. 25 independent runs of PMODE and HECO-PDE are taken on each problem and dimension respectively. The maximum function evaluations is set to $20000 \times D$, where $D$ is the dimension of an optimization problem.

\subsection{Detailed Experimental results of PMODE}

Tables 11-14 gives the experimental results of PMODE in terms of the best, median, worst, mean, standard deviation and feasibility rate of the function values $10 D, 30 D, 50 D$ and $100 D$. $c$ is the number of violated constraints at the median solution: the sequence of three numbers indicate the number of violations (including inequality and equality) by more than 1.0 , in the range $[0.01,1.0]$ and in the range $[0.0001,0.01]$ respectively. $\bar{v}$ denotes the mean value of the violations of all constraints at the median solution. SR is the feasibility rate of the solutions obtained in 25 runs. $\overline{v i o}$ denotes the mean constraint violation value of all the solutions of 25 runs.

Table 11: Function Values of PMODE Achieved for 10D $($ MaxFES $=20000 \times D)$

\begin{tabular}{|c|c|c|c|c|c|c|c|}
\hline problem & $\mathrm{C} 01$ & $\mathrm{C} 02$ & $\mathrm{C} 03$ & $\mathrm{C} 04$ & $\mathrm{C} 05$ & $\mathrm{C} 06$ & $\mathrm{C} 07$ \\
\hline best & $2.14611 \mathrm{e}-24$ & $9.11866 \mathrm{e}-25$ & $9.41726 \mathrm{e}+03$ & $3.88033 \mathrm{e}+01$ & $9.91336 \mathrm{e}-22$ & $7.39402 \mathrm{e}+01$ & $-1.60857 \mathrm{e}+02$ \\
\hline median & $5.68959 \mathrm{e}-24$ & $4.44967 \mathrm{e}-24$ & $1.71079 \mathrm{e}+04$ & $8.32015 \mathrm{e}+01$ & $3.52520 \mathrm{e}-20$ & $1.78727 \mathrm{e}+02$ & $-7.46900 \mathrm{e}+01$ \\
\hline $\mathrm{c}$ & $\begin{array}{llll}0 & 0 & 0\end{array}$ & $\begin{array}{llll}0 & 0 & 0\end{array}$ & 010 & $\begin{array}{lll}0 & 0 & 0\end{array}$ & $\begin{array}{llllllll}0 & 0 & 0\end{array}$ & 230 & 200 \\
\hline $\bar{v}$ & $0.00000 \mathrm{e}+00$ & $0.00000 \mathrm{e}+00$ & $1.16861 \mathrm{e}-02$ & $0.00000 \mathrm{e}+00$ & $0.00000 \mathrm{e}+00$ & $5.24831 \mathrm{e}-01$ & $7.57086 \mathrm{e}+01$ \\
\hline Mean & $8.63981 \mathrm{e}-24$ & $.48690 \mathrm{e}-24$ & $5.94558 \mathrm{e}+04$ & $8.13511 \mathrm{e}+01$ & $6.08489 \mathrm{e}-20$ & $2.16101 \mathrm{e}+02$ & $-5.61896 e+01$ \\
\hline Worst & $3.43159 \mathrm{e}-23$ & $1.80413 \mathrm{e}-23$ & $4.46449 \mathrm{e}+04$ & $1.02793 \mathrm{e}+02$ & $2.59659 \mathrm{e}-19$ & $1.35417 \mathrm{e}+03$ & $-9.13489 e+01$ \\
\hline std & $7.75626 \mathrm{e}-24$ & $4.92035 \mathrm{e}-24$ & $8.34239 \mathrm{e}+04$ & $1.39084 \mathrm{e}+01$ & $6.96775 \mathrm{e}-20$ & $2.51834 \mathrm{e}+02$ & $5.98942 \mathrm{e}+01$ \\
\hline SR & 100 & 100 & 4 & 100 & 100 & 16 & 4 \\
\hline$\overline{v i o}$ & $0.00000 \mathrm{e}+00$ & $0.00000 \mathrm{e}+00$ & $1.65910 \mathrm{e}-02$ & $0.00000 \mathrm{e}+00$ & $0.00000 \mathrm{e}+00$ & $5.21616 \mathrm{e}-01$ & $7.85737 \mathrm{e}+01$ \\
\hline problem & $\mathrm{C} 08$ & C09 & C10 & C11 & C12 & $\mathrm{C} 13$ & C14 \\
\hline best & $-1.34840 \mathrm{e}-03$ & $-4.97525 \mathrm{e}-03$ & $-5.09646 \mathrm{e}-04$ & $-1.62228 \mathrm{e}+02$ & $3.98790 \mathrm{e}+00$ & $5.15504 \mathrm{e}-21$ & $2.39559 \mathrm{e}+00$ \\
\hline median & $-1.34840 \mathrm{e}-03$ & $-4.97525 \mathrm{e}-03$ & $-5.09645 \mathrm{e}-04$ & $-3.70137 e+02$ & $3.98863 \mathrm{e}+00$ & $6.58037 \mathrm{e}-20$ & $3.39761 \mathrm{e}+00$ \\
\hline c & $\begin{array}{llll}0 & 0 & 0\end{array}$ & $\begin{array}{llll}0 & 0 & 0\end{array}$ & $\begin{array}{llll}0 & 0 & 0\end{array}$ & 100 & $\begin{array}{lllllll}0 & 0 & 0\end{array}$ & $\begin{array}{lll}0 & 0 & 0\end{array}$ & $\begin{array}{lll}0 & 0 & 0\end{array}$ \\
\hline $\bar{v}$ & $0.00000 \mathrm{e}+00$ & $0.00000 \mathrm{e}+00$ & $0.00000 \mathrm{e}+00$ & $2.40353 e+02$ & $0.00000 \mathrm{e}+00$ & $0.00000 \mathrm{e}+00$ & $4.58844 \mathrm{e}-06$ \\
\hline Mean & $-1.34840 \mathrm{e}-03$ & $-4.97525 \mathrm{e}-03$ & $-5.09645 \mathrm{e}-04$ & $-3.55967 e+02$ & $4.83886 \mathrm{e}+00$ & $1.59463 \mathrm{e}-01$ & $3.45367 \mathrm{e}+00$ \\
\hline Worst & $-1.34840 \mathrm{e}-03$ & $-4.97525 \mathrm{e}-03$ & $-5.09637 \mathrm{e}-04$ & $-5.43096 e+02$ & $1.46065 \mathrm{e}+01$ & $3.98658 \mathrm{e}+00$ & $3.67062 \mathrm{e}+00$ \\
\hline std & $3.25752 \mathrm{e}-10$ & $0.00000 \mathrm{e}+00$ & $2.05524 \mathrm{e}-09$ & $9.43249 \mathrm{e}+01$ & $2.87977 \mathrm{e}+00$ & 7.81207e-01 & $3.43063 \mathrm{e}-01$ \\
\hline SR & 100 & 100 & 100 & 0 & 100 & 100 & 48 \\
\hline$\overline{\text { vio }}$ & $0.00000 \mathrm{e}+00$ & $0.00000 \mathrm{e}+00$ & $0.00000 \mathrm{e}+00$ & $2.37912 \mathrm{e}+02$ & $0.00000 \mathrm{e}+00$ & $0.00000 \mathrm{e}+00$ & $5.32261 \mathrm{e}-05$ \\
\hline problem & $\mathrm{C} 15$ & C16 & C17 & C18 & C19 & $\mathrm{C} 20$ & $\mathrm{C} 21$ \\
\hline best & $5.49772 \mathrm{e}+00$ & $5.18363 \mathrm{e}+01$ & $9.97584 \mathrm{e}-01$ & $1.00000 \mathrm{e}+01$ & $0.00000 \mathrm{e}+00$ & $1.47930 \mathrm{e}+00$ & $3.98790 \mathrm{e}+00$ \\
\hline median & $1.17811 \mathrm{e}+01$ & $5.65481 \mathrm{e}+01$ & $9.14289 \mathrm{e}-01$ & $3.62209 \mathrm{e}+01$ & $0.00000 \mathrm{e}+00$ & $1.81463 \mathrm{e}+00$ & $3.98881 \mathrm{e}+00$ \\
\hline $\mathrm{c}$ & $\begin{array}{llll}0 & 0 & 1\end{array}$ & $\begin{array}{llll}0 & 0 & 1\end{array}$ & 110 & $\begin{array}{llll}0 & 1 & 0\end{array}$ & $\begin{array}{lll}1 & 0 & 0\end{array}$ & $\begin{array}{lll}0 & 0 & 0\end{array}$ & $\begin{array}{lll}0 & 0 & 0\end{array}$ \\
\hline $\bar{v}$ & $5.51058 \mathrm{e}-05$ & $1.46131 \mathrm{e}-04$ & $50540 \mathrm{e}+00$ & $1.29979 \mathrm{e}-01$ & $6.63359 \mathrm{e}+03$ & $0.00000 \mathrm{e}+00$ & $0.00000 \mathrm{e}+00$ \\
\hline Mean & $1.21578 \mathrm{e}+01$ & $6.15751 \mathrm{e}+01$ & $9.80767 \mathrm{e}-01$ & $4.10083 \mathrm{e}+01$ & $0.00000 \mathrm{e}+00$ & $1.81955 \mathrm{e}+00$ & $5.46111 \mathrm{e}+00$ \\
\hline Worst & $8.63678 \mathrm{e}+00$ & $5.65464 \mathrm{e}+01$ & $1.00244 \mathrm{e}+00$ & $5.17217 \mathrm{e}+01$ & $0.00000 \mathrm{e}+00$ & $2.17692 \mathrm{e}+00$ & $2.27853 \mathrm{e}+01$ \\
\hline std & $3.90569 \mathrm{e}+00$ & $8.31210 \mathrm{e}+00$ & $5.62635 \mathrm{e}-02$ & $1.44737 \mathrm{e}+01$ & $0.00000 \mathrm{e}+00$ & $1.76447 \mathrm{e}-01$ & $4.99452 \mathrm{e}+00$ \\
\hline $\mathrm{SR}$ & 40 & 12 & 0 & 4 & 0 & 100 & 100 \\
\hline$\overline{v i o}$ & $3.24071 \mathrm{e}-04$ & $1.99125 \mathrm{e}-04$ & $5.50824 \mathrm{e}+00$ & $2.93346 \mathrm{e}+00$ & $6.63359 \mathrm{e}+03$ & $0.00000 \mathrm{e}+00$ & $0.00000 \mathrm{e}+00$ \\
\hline problem & $\mathrm{C} 22$ & $\mathrm{C} 23$ & $\mathrm{C} 24$ & $\mathrm{C} 25$ & $\mathrm{C} 26$ & $\mathrm{C} 27$ & $\mathrm{C} 28$ \\
\hline best & $4.75166 \mathrm{e}-21$ & $40531 \mathrm{e}+00$ & $5.49772 \mathrm{e}+00$ & $5.65486 \mathrm{e}+01$ & $1.51776 \mathrm{e}-01$ & $6.28153 \mathrm{e}+01$ & $0.00000 \mathrm{e}+00$ \\
\hline median & $1.04790 \mathrm{e}-19$ & $3.81500 \mathrm{e}+00$ & $1.49229 \mathrm{e}+01$ & $4.39817 \mathrm{e}+01$ & $1.00954 \mathrm{e}+00$ & $1.37228 \mathrm{e}+01$ & $0.00000 \mathrm{e}+00$ \\
\hline c & $\begin{array}{lllll}0 & 0 & 0\end{array}$ & $\begin{array}{llll}0 & 0 & 0\end{array}$ & $\begin{array}{llll}0 & 0 & 1\end{array}$ & $\begin{array}{lllllll}0 & 0 & 1\end{array}$ & 101 & $\begin{array}{lll}0 & 2 & 0\end{array}$ & 100 \\
\hline $\bar{v}$ & $0.00000 \mathrm{e}+00$ & $00000 e+00$ & $2.11608 \mathrm{e}-04$ & $1.46534 \mathrm{e}-04$ & $5.50411 \mathrm{e}+00$ & $2.76359 \mathrm{e}-01$ & $6.63359 \mathrm{e}+03$ \\
\hline Mean & $2.88192 \mathrm{e}-19$ & $3.29617 \mathrm{e}+00$ & $1.37914 \mathrm{e}+01$ & $6.26432 \mathrm{e}+01$ & $9.08549 \mathrm{e}-01$ & $2.33382 \mathrm{e}+01$ & $1.62221 \mathrm{e}+00$ \\
\hline Worst & $2.27974 \mathrm{e}-18$ & $3.31445 \mathrm{e}+00$ & $1.49199 \mathrm{e}+01$ & $7.53947 \mathrm{e}+01$ & $1.00543 \mathrm{e}+00$ & $2.82342 \mathrm{e}+00$ & $6.37173 \mathrm{e}+00$ \\
\hline std & $5.20942 \mathrm{e}-19$ & $4.03648 \mathrm{e}-01$ & $3.65075 \mathrm{e}+00$ & $1.19573 \mathrm{e}+01$ & $2.12119 \mathrm{e}-01$ & $2.20528 \mathrm{e}+01$ & $2.92278 \mathrm{e}+00$ \\
\hline SR & 100 & 52 & 20 & 4 & 0 & 0 & 0 \\
\hline$\overline{v i o}$ & $0.00000 \mathrm{e}+00$ & $6.82870 \mathrm{e}-05$ & $4.23089 \mathrm{e}-04$ & $2.31590 \mathrm{e}-04$ & $5.46639 \mathrm{e}+00$ & $2.48248 \mathrm{e}-01$ & $6.63622 \mathrm{e}+03$ \\
\hline
\end{tabular}

\subsection{Experimental Results of HECO-PDE}

Tables 15-18 shows the experimental results of HECO-PDE in terms of the best, median, worst, mean, standard deviation and feasibility rate of the function values $10 D, 30 D, 50 D$ and $100 D$. $c$ is the number of violated constraints at the median solution: the sequence of three numbers indicate the number of violations (including inequality and equality) by more than 1.0 , in the range $[0.01,1.0]$ and in the range $[0.0001,0.01]$ respectively. $\bar{v}$ denotes the mean value of the violations of all constraints at the median solution. SR is the 
Table 12: Function Values of PMODE Achieved for 30D $($ MaxFES $=20000 \times D)$

\begin{tabular}{|c|c|c|c|c|c|c|c|}
\hline problem & $\mathrm{C} 01$ & $\mathrm{C} 02$ & $\mathrm{C} 03$ & $\mathrm{C} 04$ & $\mathrm{C} 05$ & C06 & $\mathrm{C} 07$ \\
\hline best & $8.45246 \mathrm{e}+00$ & $1.31645 \mathrm{e}+00$ & $1.21533 \mathrm{e}+05$ & $1.62387 \mathrm{e}+02$ & $2.37034 \mathrm{e}+01$ & $8.68151 \mathrm{e}+02$ & $-7.78047 \mathrm{e}+01$ \\
\hline median & $6.22106 \mathrm{e}+01$ & $6.14986 \mathrm{e}+01$ & $4.46899 \mathrm{e}+05$ & $3.66171 \mathrm{e}+02$ & $1.22896 \mathrm{e}+02$ & $2.23162 \mathrm{e}+03$ & $-1.15271 \mathrm{e}+02$ \\
\hline $\mathrm{c}$ & $\begin{array}{lll}0 & 0 & 0\end{array}$ & 000 & 010 & 000 & 000 & 410 & 200 \\
\hline $\bar{v}$ & $0.00000 \mathrm{e}+00$ & $0.00000 \mathrm{e}+00$ & $1.56053 \mathrm{e}-02$ & $0.00000 \mathrm{e}+00$ & $0.00000 \mathrm{e}+00$ & $3.04630 \mathrm{e}+00$ & $1.40266 \mathrm{e}+03$ \\
\hline Mean & $1.01581 \mathrm{e}+02$ & $8.79346 e+01$ & $5.24967 \mathrm{e}+05$ & $3.25711 \mathrm{e}+02$ & $1.76987 \mathrm{e}+02$ & $2.75522 \mathrm{e}+03$ & $-6.32051 e+01$ \\
\hline Worst & $3.13217 \mathrm{e}+02$ & $5.79893 \mathrm{e}+02$ & $2.19709 \mathrm{e}+05$ & $3.91920 \mathrm{e}+02$ & $8.55390 \mathrm{e}+02$ & $1.96412 \mathrm{e}+03$ & $-1.85538 \mathrm{e}+02$ \\
\hline std & $8.75557 \mathrm{e}+01$ & $1.15326 \mathrm{e}+02$ & $4.36345 \mathrm{e}+05$ & $7.78934 \mathrm{e}+01$ & $1.76071 \mathrm{e}+02$ & $1.34597 \mathrm{e}+03$ & $8.25769 \mathrm{e}+01$ \\
\hline SR & 100 & 100 & 8 & 100 & 100 & 0 & 0 \\
\hline$\overline{v i o}$ & $0.00000 \mathrm{e}+00$ & $0.00000 \mathrm{e}+00$ & $2.97523 \mathrm{e}-02$ & $0.00000 \mathrm{e}+00$ & $0.00000 \mathrm{e}+00$ & $3.07096 \mathrm{e}+00$ & $1.37837 \mathrm{e}+03$ \\
\hline problem & $\mathrm{C} 08$ & $\mathrm{C} 09$ & C10 & C11 & C12 & C13 & C14 \\
\hline best & $4.30883 \mathrm{e}+00$ & $-2.66551 e-03$ & $2.93934 \mathrm{e}+00$ & $-1.95483 e+03$ & $2.58898 \mathrm{e}+02$ & $7.91928 \mathrm{e}+05$ & $1.16005 \mathrm{e}+01$ \\
\hline median & $8.11294 \mathrm{e}+00$ & $1.04331 \mathrm{e}+00$ & $1.12307 \mathrm{e}+00$ & $-2.39334 \mathrm{e}+03$ & $8.71198 \mathrm{e}+02$ & $4.03949 \mathrm{e}+06$ & $1.73876 \mathrm{e}+01$ \\
\hline $\mathrm{c}$ & 200 & $\begin{array}{lll}0 & 0 & 0\end{array}$ & 200 & 100 & 100 & 100 & 200 \\
\hline $\bar{v}$ & $2.27078 \mathrm{e}+02$ & $0.00000 \mathrm{e}+00$ & $9.07387 \mathrm{e}+04$ & $6.74605 \mathrm{e}+02$ & $3.93445 \mathrm{e}+02$ & $2.83340 \mathrm{e}+02$ & $2.44359 \mathrm{e}+03$ \\
\hline Mean & $9.09124 \mathrm{e}+00$ & $1.22155 \mathrm{e}+00$ & $5.79186 \mathrm{e}+00$ & $-1.58495 e+03$ & $9.71512 \mathrm{e}+02$ & $1.01176 \mathrm{e}+07$ & $1.69531 \mathrm{e}+01$ \\
\hline Worst & $1.59694 \mathrm{e}+01$ & $5.12011 \mathrm{e}+00$ & $8.62571 \mathrm{e}+00$ & $-1.61106 \mathrm{e}+03$ & $3.21002 \mathrm{e}+03$ & $6.20270 \mathrm{e}+07$ & $1.95298 \mathrm{e}+01$ \\
\hline std & $3.15098 \mathrm{e}+00$ & $1.49632 \mathrm{e}+00$ & $3.96077 \mathrm{e}+00$ & $5.24220 \mathrm{e}+02$ & $5.76453 \mathrm{e}+02$ & $1.34472 \mathrm{e}+07$ & $1.88152 \mathrm{e}+00$ \\
\hline SR & 0 & 60 & 0 & 0 & 0 & 0 & 0 \\
\hline$\overline{v i o}$ & $2.63526 \mathrm{e}+02$ & $4.79756 \mathrm{e}-02$ & $1.04036 \mathrm{e}+05$ & $8.29760 \mathrm{e}+02$ & $4.46996 \mathrm{e}+02$ & $3.57934 \mathrm{e}+02$ & $2.53791 \mathrm{e}+03$ \\
\hline problem & C15 & C16 & C17 & C18 & C19 & C20 & C21 \\
\hline best & $1.17809 \mathrm{e}+01$ & $2.01062 \mathrm{e}+02$ & $1.02982 \mathrm{e}+00$ & $2.55074 \mathrm{e}+02$ & $4.15257 \mathrm{e}+01$ & $8.22588 \mathrm{e}+00$ & $2.89044 \mathrm{e}+02$ \\
\hline median & $1.49226 \mathrm{e}+01$ & $2.08916 \mathrm{e}+02$ & $1.15328 \mathrm{e}+00$ & $8.36250 \mathrm{e}+02$ & $4.02094 \mathrm{e}+01$ & $9.25366 \mathrm{e}+00$ & $7.53484 \mathrm{e}+02$ \\
\hline $\mathrm{c}$ & $\begin{array}{lll}0 & 0 & 0\end{array}$ & $\begin{array}{lll}0 & 0 & 1\end{array}$ & 200 & 100 & 100 & $\begin{array}{lll}0 & 0 & 0\end{array}$ & 100 \\
\hline $\bar{v}$ & $9.70150 \mathrm{e}-06$ & $7.20124 \mathrm{e}-05$ & $2.62061 \mathrm{e}+02$ & $4.75943 \mathrm{e}+05$ & $2.14626 \mathrm{e}+04$ & $0.00000 \mathrm{e}+00$ & $3.14773 \mathrm{e}+02$ \\
\hline Mean & $1.81898 \mathrm{e}+$ & $2.11052 \mathrm{e}-$ & $1.17571 \mathrm{e}$ & $9.55141 \mathrm{e}$ & $5.60410 \mathrm{e}$ & $9.23266 \mathrm{e}+00$ & $7.69190 \mathrm{e}+02$ \\
\hline Worst & $2.12045 \mathrm{e}+01$ & $2.01059 \mathrm{e}+02$ & $1.45716 \mathrm{e}+00$ & $2.05000 \mathrm{e}+03$ & $8.07226 \mathrm{e}+01$ & $1.00181 \mathrm{e}+01$ & $1.32907 \mathrm{e}+03$ \\
\hline std & $2.73591 \mathrm{e}+00$ & $1.23599 \mathrm{e}+01$ & $1.00234 \mathrm{e}-01$ & $4.97848 \mathrm{e}+02$ & $1.26095 \mathrm{e}+01$ & $4.38197 \mathrm{e}-01$ & $2.77606 \mathrm{e}+02$ \\
\hline SR & 48 & 16 & 0 & 0 & 0 & 100 & 0 \\
\hline$\overline{v i o}$ & $1.84991 \mathrm{e}-04$ & $1.85297 \mathrm{e}-04$ & $3.06938 \mathrm{e}+02$ & $9.84241 \mathrm{e}+05$ & $2.14638 \mathrm{e}+04$ & $0.00000 \mathrm{e}+00$ & $3.45572 \mathrm{e}+02$ \\
\hline problem & $\mathrm{C} 22$ & $\mathrm{C} 23$ & $\mathrm{C} 24$ & $\mathrm{C} 25$ & C26 & C27 & C28 \\
\hline best & $2.62086 \mathrm{e}+06$ & $1.90652 \mathrm{e}+01$ & $1.49225 \mathrm{e}+01$ & $1.90066 \mathrm{e}+02$ & $1.21225 \mathrm{e}+00$ & $9.75533 \mathrm{e}+02$ & $6.22618 \mathrm{e}+01$ \\
\hline median & $2.21334 \mathrm{e}+07$ & $2.03930 \mathrm{e}+01$ & $1.80597 \mathrm{e}+01$ & $2.01061 \mathrm{e}+02$ & $1.59385 \mathrm{e}+00$ & $5.32941 \mathrm{e}+03$ & $7.70427 \mathrm{e}+01$ \\
\hline $\mathrm{c}$ & 200 & 200 & $\begin{array}{lll}0 & 0 & 1\end{array}$ & $\begin{array}{llll}0 & 0 & 1\end{array}$ & 200 & 100 & 100 \\
\hline $\bar{v}$ & $6.24786 \mathrm{e}+02$ & $1.24452 \mathrm{e}+04$ & $3.08672 \mathrm{e}-03$ & $1.07590 \mathrm{e}-04$ & $1.14320 \mathrm{e}+03$ & $4.62537 \mathrm{e}+05$ & $2.14813 \mathrm{e}+04$ \\
\hline Mean & $4.79527 \mathrm{e}+07$ & $2.04905 \mathrm{e}+01$ & $1.89402 \mathrm{e}+01$ & $2.36413 \mathrm{e}+02$ & $1.65233 \mathrm{e}+00$ & $3.53610 \mathrm{e}+03$ & $9.64144 \mathrm{e}+01$ \\
\hline Worst & $3.09761 \mathrm{e}+08$ & $2.13998 \mathrm{e}+01$ & $2.61751 \mathrm{e}+01$ & $3.56724 \mathrm{e}+02$ & $2.35945 \mathrm{e}+00$ & $7.53113 \mathrm{e}+03$ & $1.69217 \mathrm{e}+02$ \\
\hline std & $6.33025 \mathrm{e}+07$ & $7.08163 \mathrm{e}-01$ & $2.51595 \mathrm{e}+00$ & $3.83776 \mathrm{e}+01$ & $3.21242 \mathrm{e}-01$ & $1.96683 \mathrm{e}+03$ & $2.58908 \mathrm{e}+01$ \\
\hline SR & 0 & 0 & 36 & 28 & 0 & 0 & 0 \\
\hline$\overline{v i o}$ & $7.67227 \mathrm{e}+02$ & $1.37688 \mathrm{e}+04$ & $1.47415 \mathrm{e}+02$ & $2.75679 \mathrm{e}+02$ & $1.26016 \mathrm{e}+03$ & $9.43760 \mathrm{e}+05$ & $2.14813 e+04$ \\
\hline
\end{tabular}

feasibility rate of the solutions obtained in 25 runs. $\overline{v i o}$ denotes the mean constraint violation value of all the solutions of 25 runs. 
Table 13: Function Values of PMODE Achieved for 50D $($ MaxFES $=20000 \times D)$

\begin{tabular}{|c|c|c|c|c|c|c|c|}
\hline problem & $\mathrm{C} 01$ & $\mathrm{C} 02$ & $\mathrm{C} 03$ & $\mathrm{C} 04$ & C05 & C06 & C07 \\
\hline best & $2.28064 \mathrm{e}+03$ & $1.98396 \mathrm{e}+03$ & $1.93920 \mathrm{e}+05$ & $3.55349 \mathrm{e}+02$ & $5.97421 \mathrm{e}+03$ & $1.75834 \mathrm{e}+03$ & $-5.36955 e+02$ \\
\hline median & $4.52311 \mathrm{e}+03$ & $3.66519 \mathrm{e}+03$ & $8.18781 \mathrm{e}+06$ & $4.77077 \mathrm{e}+02$ & $1.92933 \mathrm{e}+04$ & $8.24012 \mathrm{e}+03$ & $-1.18883 e+02$ \\
\hline $\mathrm{c}$ & $\begin{array}{lll}0 & 0 & 0\end{array}$ & $\begin{array}{lllllllll}0 & 0 & 0\end{array}$ & 010 & 000 & $\begin{array}{lll}0 & 0 & 0\end{array}$ & 310 & 200 \\
\hline $\bar{v}$ & $0.00000 \mathrm{e}+00$ & $0.00000 \mathrm{e}+00$ & $2.11433 \mathrm{e}-02$ & $0.00000 \mathrm{e}+00$ & $0.00000 \mathrm{e}+00$ & $3.67257 \mathrm{e}+00$ & $2.89321 \mathrm{e}+03$ \\
\hline Mean & $4.57826 \mathrm{e}+03$ & $3.86686 \mathrm{e}+03$ & $2.56010 \mathrm{e}+06$ & $5.28980 \mathrm{e}+02$ & $2.07483 \mathrm{e}+04$ & $5.21220 \mathrm{e}+03$ & $-1.52570 \mathrm{e}+02$ \\
\hline Worst & $6.58848 \mathrm{e}+03$ & $5.80477 \mathrm{e}+03$ & $1.80087 \mathrm{e}+06$ & $6.71820 \mathrm{e}+02$ & $6.05995 \mathrm{e}+04$ & $4.84112 \mathrm{e}+03$ & $-2.33206 e+02$ \\
\hline std & $9.65902 \mathrm{e}+02$ & $9.24684 \mathrm{e}+02$ & $3.45818 \mathrm{e}+06$ & $1.19169 \mathrm{e}+02$ & $1.16951 \mathrm{e}+04$ & $2.34983 \mathrm{e}+03$ & $1.35106 \mathrm{e}+02$ \\
\hline SR & 100 & 100 & 8 & 100 & 100 & 16 & 0 \\
\hline$\overline{v i o}$ & $0.00000 \mathrm{e}+00$ & $0.00000 \mathrm{e}+00$ & $4.21099 \mathrm{e}-02$ & $0.00000 \mathrm{e}+00$ & $0.00000 \mathrm{e}+00$ & $3.45598 \mathrm{e}+00$ & $2.82329 \mathrm{e}+03$ \\
\hline problem & $\mathrm{C} 08$ & C09 & $\mathrm{C} 10$ & C11 & C12 & C13 & C14 \\
\hline best & $1.02717 \mathrm{e}+01$ & $5.74202 \mathrm{e}+00$ & $1.74030 \mathrm{e}+01$ & $-1.47463 e+03$ & $3.75936 \mathrm{e}+03$ & $4.56714 \mathrm{e}+07$ & $1.83443 \mathrm{e}+01$ \\
\hline median & $1.47237 \mathrm{e}+01$ & $6.01563 \mathrm{e}+00$ & $2.35321 \mathrm{e}+01$ & $-1.04457 \mathrm{e}+03$ & $6.65909 \mathrm{e}+03$ & $1.81715 \mathrm{e}+08$ & $1.96039 \mathrm{e}+01$ \\
\hline c & 200 & 100 & 200 & 100 & 100 & 200 & 200 \\
\hline $\bar{v}$ & $1.36576 \mathrm{e}+03$ & $1.25530 \mathrm{e}+02$ & $2.42791 \mathrm{e}+06$ & $4.02216 \mathrm{e}+03$ & $3.25068 \mathrm{e}+03$ & $2.07518 \mathrm{e}+03$ & $1.03922 \mathrm{e}+04$ \\
\hline Mean & $1.67054 \mathrm{e}+01$ & $7.26445 \mathrm{e}+00$ & $1.77968 \mathrm{e}+01$ & $-1.28194 \mathrm{e}+03$ & $7.21543 \mathrm{e}+03$ & $1.91988 \mathrm{e}+08$ & $1.96436 \mathrm{e}+01$ \\
\hline Worst & $2.12763 \mathrm{e}+01$ & $9.49550 \mathrm{e}+00$ & $1.47180 \mathrm{e}+01$ & $-9.95573 \mathrm{e}+02$ & $1.61630 \mathrm{e}+04$ & $6.34024 \mathrm{e}+08$ & $2.09103 \mathrm{e}+01$ \\
\hline std & $3.86802 \mathrm{e}+00$ & $1.35384 \mathrm{e}+00$ & $5.56564 \mathrm{e}+00$ & $3.36372 \mathrm{e}+02$ & $2.58950 \mathrm{e}+03$ & $1.28596 \mathrm{e}+08$ & $6.79060 \mathrm{e}-01$ \\
\hline $\mathrm{SR}$ & 0 & 0 & 0 & 0 & 0 & 0 & 0 \\
\hline$\overline{v i o}$ & $1.34029 \mathrm{e}+03$ & $2.09972 \mathrm{e}+02$ & $2.50252 \mathrm{e}+06$ & $4.14513 \mathrm{e}+03$ & $3.50114 \mathrm{e}+03$ & $2.07567 \mathrm{e}+03$ & $1.00248 \mathrm{e}+04$ \\
\hline problem & $\mathrm{C} 15$ & C16 & $\mathrm{C} 17$ & C18 & C19 & $\mathrm{C} 20$ & $\mathrm{C} 21$ \\
\hline best & $1.80641 \mathrm{e}+01$ & $4.08407 \mathrm{e}+02$ & $1.81562 \mathrm{e}+00$ & $4.67375 \mathrm{e}+03$ & $1.25353 \mathrm{e}+02$ & $1.62035 \mathrm{e}+01$ & $3.46898 \mathrm{e}+03$ \\
\hline median & $2.23511 \mathrm{e}+01$ & $4.03140 \mathrm{e}+02$ & $2.69752 \mathrm{e}+00$ & $1.04115 \mathrm{e}+04$ & $1.35750 \mathrm{e}+02$ & $1.75255 \mathrm{e}+01$ & $7.01829 \mathrm{e}+03$ \\
\hline $\mathrm{c}$ & 200 & 110 & 200 & 200 & 100 & $\begin{array}{lll}0 & 0 & 0\end{array}$ & 100 \\
\hline $\bar{v}$ & $7.95359 \mathrm{e}+02$ & $5.63050 \mathrm{e}+02$ & $3.32053 e+03$ & $5.38874 \mathrm{e}+07$ & $3.63004 \mathrm{e}+04$ & $0.00000 \mathrm{e}+00$ & $3.40038 \mathrm{e}+03$ \\
\hline Mean & $2.41366 \mathrm{e}+01$ & $4.36228 \mathrm{e}+02$ & $2.61806 \mathrm{e}+00$ & $8.17305 \mathrm{e}+03$ & $1.28788 \mathrm{e}+02$ & $1.74999 \mathrm{e}+01$ & $6.90454 \mathrm{e}+03$ \\
\hline Worst & $2.81105 \mathrm{e}+01$ & $6.32413 \mathrm{e}+02$ & $3.56693 \mathrm{e}+00$ & $9.97025 \mathrm{e}+03$ & $1.40672 \mathrm{e}+02$ & $1.87708 \mathrm{e}+01$ & $1.08995 \mathrm{e}+04$ \\
\hline std & $5.05616 \mathrm{e}+00$ & $6.03201 \mathrm{e}+01$ & $3.83810 \mathrm{e}-01$ & $2.02373 \mathrm{e}+03$ & $1.62059 \mathrm{e}+01$ & $5.63727 \mathrm{e}-01$ & $1.86784 \mathrm{e}+03$ \\
\hline SR & 8 & 4 & 0 & 0 & 0 & 100 & 0 \\
\hline$\overline{v i o}$ & $9.94883 e+02$ & $8.85680 \mathrm{e}+02$ & $3.16162 \mathrm{e}+03$ & $5.42517 \mathrm{e}+07$ & $3.62998 \mathrm{e}+04$ & $0.00000 \mathrm{e}+00$ & $3.34437 \mathrm{e}+03$ \\
\hline problem & $\mathrm{C} 22$ & $\mathrm{C} 23$ & $\mathrm{C} 24$ & $\mathrm{C} 25$ & C26 & C27 & $\mathrm{C} 28$ \\
\hline best & $6.58082 \mathrm{e}+08$ & $2.09123 \mathrm{e}+01$ & $3.70265 \mathrm{e}+01$ & $5.58189 \mathrm{e}+02$ & $4.31932 \mathrm{e}+00$ & $1.50354 \mathrm{e}+04$ & $1.72173 \mathrm{e}+02$ \\
\hline median & $2.09933 \mathrm{e}+09$ & $2.14984 \mathrm{e}+01$ & $4.47071 \mathrm{e}+01$ & $7.44848 \mathrm{e}+02$ & $6.25467 \mathrm{e}+00$ & $2.78702 \mathrm{e}+04$ & $2.02544 \mathrm{e}+02$ \\
\hline $\mathrm{c}$ & 200 & 200 & 200 & 200 & 200 & 200 & 100 \\
\hline $\bar{v}$ & $7.26657 \mathrm{e}+03$ & $4.93000 \mathrm{e}+04$ & $9.13401 \mathrm{e}+03$ & $7.52269 \mathrm{e}+03$ & $1.04348 \mathrm{e}+04$ & $4.85357 \mathrm{e}+07$ & $3.63275 \mathrm{e}+04$ \\
\hline Mean & $2.02531 \mathrm{e}+09$ & $2.13180 \mathrm{e}+01$ & $4.55004 \mathrm{e}+01$ & $7.72193 \mathrm{e}+02$ & $6.51825 \mathrm{e}+00$ & $2.64735 \mathrm{e}+04$ & $2.12540 \mathrm{e}+02$ \\
\hline Worst & $4.14934 \mathrm{e}+09$ & $2.14182 \mathrm{e}+01$ & $7.05414 \mathrm{e}+01$ & $9.87128 \mathrm{e}+02$ & $9.74742 \mathrm{e}+00$ & $5.25566 \mathrm{e}+04$ & $3.08671 \mathrm{e}+02$ \\
\hline std & $7.91057 \mathrm{e}+08$ & $2.26072 \mathrm{e}-01$ & $9.58023 \mathrm{e}+00$ & $1.16580 \mathrm{e}+02$ & $1.46486 \mathrm{e}+00$ & $7.94598 \mathrm{e}+03$ & $3.13167 \mathrm{e}+01$ \\
\hline SR & 0 & 0 & 0 & 0 & 0 & 0 & 0 \\
\hline$\overline{v i o}$ & $6.99710 \mathrm{e}+03$ & $4.94889 \mathrm{e}+04$ & $9.41546 \mathrm{e}+03$ & $7.98474 \mathrm{e}+03$ & $1.09620 \mathrm{e}+04$ & $5.85456 \mathrm{e}+07$ & $3.63287 \mathrm{e}+04$ \\
\hline
\end{tabular}


Table 14: Function Values of PMODE Achieved for 100D $($ MaxFES $=20000 \times D)$

\begin{tabular}{|c|c|c|c|c|c|c|c|}
\hline problem & $\mathrm{C} 01$ & $\mathrm{C} 02$ & $\mathrm{C} 03$ & $\mathrm{C} 04$ & C05 & C06 & C07 \\
\hline best & $2.19725 \mathrm{e}+04$ & $3.08252 \mathrm{e}+04$ & $8.81317 \mathrm{e}+05$ & $8.80889 \mathrm{e}+02$ & $1.74982 \mathrm{e}+05$ & $4.28539 \mathrm{e}+03$ & $-5.36330 e+01$ \\
\hline median & $3.48956 \mathrm{e}+04$ & $4.21954 \mathrm{e}+04$ & $1.64864 \mathrm{e}+07$ & $1.05770 \mathrm{e}+03$ & $3.53987 \mathrm{e}+05$ & $1.18699 \mathrm{e}+04$ & $-3.22051 \mathrm{e}+02$ \\
\hline $\mathrm{c}$ & $\begin{array}{lll}0 & 0 & 0\end{array}$ & $\begin{array}{lllllllll}0 & 0 & 0\end{array}$ & 010 & 0000 & $\begin{array}{lll}0 & 0 & 0\end{array}$ & 500 & 200 \\
\hline $\bar{v}$ & $0.00000 \mathrm{e}+00$ & $0.00000 \mathrm{e}+00$ & $4.56214 \mathrm{e}-02$ & $0.00000 \mathrm{e}+00$ & $0.00000 \mathrm{e}+00$ & $6.78371 \mathrm{e}+00$ & $6.86213 \mathrm{e}+03$ \\
\hline Mean & $3.48826 \mathrm{e}+04$ & $4.22447 \mathrm{e}+04$ & $4.43083 \mathrm{e}+06$ & $1.07373 \mathrm{e}+03$ & $3.44449 \mathrm{e}+05$ & $1.10830 \mathrm{e}+04$ & $-1.49642 \mathrm{e}+02$ \\
\hline Worst & $4.50649 \mathrm{e}+04$ & $5.53684 \mathrm{e}+04$ & $1.44167 \mathrm{e}+07$ & $1.40462 \mathrm{e}+03$ & $5.35239 \mathrm{e}+05$ & $5.70569 \mathrm{e}+03$ & $-9.51640 \mathrm{e}+01$ \\
\hline std & $6.46300 \mathrm{e}+03$ & $6.58780 \mathrm{e}+03$ & $3.88766 \mathrm{e}+06$ & $1.34842 \mathrm{e}+02$ & $8.99856 \mathrm{e}+04$ & $4.10730 \mathrm{e}+03$ & $1.92704 \mathrm{e}+02$ \\
\hline SR & 100 & 100 & 8 & 100 & 100 & 8 & 0 \\
\hline$\overline{v i o}$ & $0.00000 \mathrm{e}+00$ & $0.00000 \mathrm{e}+00$ & $6.03512 \mathrm{e}-02$ & $0.00000 \mathrm{e}+00$ & $0.00000 \mathrm{e}+00$ & $6.29700 \mathrm{e}+00$ & $6.90888 \mathrm{e}+03$ \\
\hline problem & $\mathrm{C} 08$ & C09 & $\mathrm{C} 10$ & C11 & C12 & C13 & C14 \\
\hline best & $2.07891 \mathrm{e}+01$ & $1.02028 \mathrm{e}+01$ & $3.48310 \mathrm{e}+01$ & $-1.42141 \mathrm{e}+03$ & $3.13441 \mathrm{e}+04$ & $1.56586 \mathrm{e}+09$ & $2.07419 \mathrm{e}+01$ \\
\hline median & $2.76848 \mathrm{e}+01$ & $8.33249 \mathrm{e}+00$ & $3.71183 \mathrm{e}+01$ & $-9.50141 \mathrm{e}+02$ & $3.75117 \mathrm{e}+04$ & $3.12784 \mathrm{e}+09$ & $2.12499 \mathrm{e}+01$ \\
\hline c & 200 & 100 & 200 & 100 & 100 & 100 & 200 \\
\hline $\bar{v}$ & $7.53044 \mathrm{e}+03$ & $6.19357 \mathrm{e}+03$ & $4.52030 \mathrm{e}+07$ & $1.53424 \mathrm{e}+04$ & $1.84512 \mathrm{e}+04$ & $1.19932 \mathrm{e}+04$ & $5.14897 \mathrm{e}+04$ \\
\hline Mean & $2.57686 \mathrm{e}+01$ & $9.37371 \mathrm{e}+00$ & $3.81451 \mathrm{e}+01$ & $-1.44764 \mathrm{e}+03$ & $3.79194 \mathrm{e}+04$ & $3.21154 \mathrm{e}+09$ & $2.12221 \mathrm{e}+01$ \\
\hline Worst & $3.55284 \mathrm{e}+01$ & $9.85473 \mathrm{e}+00$ & $3.92237 \mathrm{e}+01$ & $-1.87066 e+03$ & $4.90522 \mathrm{e}+04$ & $5.25677 \mathrm{e}+09$ & $2.12777 \mathrm{e}+01$ \\
\hline std & $4.91663 \mathrm{e}+00$ & $8.36433 \mathrm{e}-01$ & $5.42529 \mathrm{e}+00$ & $2.32202 \mathrm{e}+02$ & $4.38064 \mathrm{e}+03$ & $9.73355 \mathrm{e}+08$ & $1.97632 \mathrm{e}-01$ \\
\hline $\mathrm{SR}$ & 0 & 0 & 0 & 0 & 0 & 0 & 0 \\
\hline$\overline{v i o}$ & $7.58180 \mathrm{e}+03$ & $6.67623 \mathrm{e}+03$ & $4.67914 \mathrm{e}+07$ & $1.58347 \mathrm{e}+04$ & $1.86740 \mathrm{e}+04$ & $1.22397 \mathrm{e}+04$ & $5.20788 \mathrm{e}+04$ \\
\hline problem & C15 & C16 & C17 & C18 & C19 & C20 & $\mathrm{C} 21$ \\
\hline best & $3.77923 \mathrm{e}+01$ & $1.29718 \mathrm{e}+03$ & $6.87595 \mathrm{e}+00$ & $3.08885 \mathrm{e}+04$ & $2.94193 \mathrm{e}+02$ & $3.74545 \mathrm{e}+01$ & $2.74753 \mathrm{e}+04$ \\
\hline median & $3.78513 \mathrm{e}+01$ & $1.49338 \mathrm{e}+03$ & $1.01553 \mathrm{e}+01$ & $3.87156 \mathrm{e}+04$ & $3.56124 \mathrm{e}+02$ & $3.90835 \mathrm{e}+01$ & $3.62471 \mathrm{e}+04$ \\
\hline $\mathrm{c}$ & 200 & 200 & 200 & 200 & 100 & $\begin{array}{lll}0 & 0 & 0\end{array}$ & 100 \\
\hline $\bar{v}$ & $1.52068 \mathrm{e}+04$ & $.30303 e+04$ & $1.81611 \mathrm{e}+04$ & $1.06212 \mathrm{e}+09$ & $7.33963 \mathrm{e}+04$ & $0.00000 \mathrm{e}+00$ & $1.78372 \mathrm{e}+04$ \\
\hline Mean & $4.32079 \mathrm{e}+01$ & $1.50611 \mathrm{e}+03$ & $9.95507 \mathrm{e}+00$ & $4.00109 \mathrm{e}+04$ & $3.50146 \mathrm{e}+02$ & $3.90733 \mathrm{e}+01$ & $3.58695 \mathrm{e}+04$ \\
\hline Worst & $5.12716 \mathrm{e}+01$ & $1.65646 \mathrm{e}+03$ & $1.20672 \mathrm{e}+01$ & $4.21590 \mathrm{e}+04$ & $4.24377 \mathrm{e}+02$ & $4.02646 \mathrm{e}+01$ & $4.50014 \mathrm{e}+04$ \\
\hline std & $3.66450 \mathrm{e}+00$ & $1.00916 \mathrm{e}+02$ & $1.27062 \mathrm{e}+00$ & $3.97574 \mathrm{e}+03$ & $2.95260 \mathrm{e}+01$ & $7.19816 \mathrm{e}-01$ & $4.72648 \mathrm{e}+03$ \\
\hline SR & 0 & 0 & 0 & 0 & 0 & 100 & 0 \\
\hline$\overline{v i o}$ & $1.49949 \mathrm{e}+04$ & $1.33886 \mathrm{e}+04$ & $1.77606 \mathrm{e}+04$ & $1.03625 \mathrm{e}+09$ & $7.33968 \mathrm{e}+04$ & $0.00000 \mathrm{e}+00$ & $1.76425 \mathrm{e}+04$ \\
\hline problem & $\mathrm{C} 22$ & $\mathrm{C} 23$ & $\mathrm{C} 24$ & $\mathrm{C} 25$ & $\mathrm{C} 26$ & C27 & $\mathrm{C} 28$ \\
\hline best & $1.61392 \mathrm{e}+10$ & $2.14640 \mathrm{e}+01$ & $6.86652 \mathrm{e}+01$ & $2.11668 \mathrm{e}+03$ & $2.04175 \mathrm{e}+01$ & $9.99239 \mathrm{e}+04$ & $3.98592 \mathrm{e}+02$ \\
\hline median & $3.19578 \mathrm{e}+10$ & $2.15640 \mathrm{e}+01$ & $7.90501 \mathrm{e}+01$ & $2.58110 \mathrm{e}+03$ & $2.85646 \mathrm{e}+01$ & $1.39773 \mathrm{e}+05$ & $5.50789 \mathrm{e}+02$ \\
\hline $\mathrm{c}$ & 200 & 200 & 200 & 200 & 200 & 200 & 100 \\
\hline $\bar{v}$ & $3.76342 \mathrm{e}+04$ & $.88806 \mathrm{e}+05$ & $5.61628 \mathrm{e}+04$ & $4.98537 \mathrm{e}+04$ & $5.49798 \mathrm{e}+04$ & $1.06865 \mathrm{e}+09$ & $7.34298 \mathrm{e}+04$ \\
\hline Mean & $3.42906 \mathrm{e}+10$ & $2.14957 \mathrm{e}+01$ & $7.90906 \mathrm{e}+01$ & $2.52591 \mathrm{e}+03$ & $2.92427 \mathrm{e}+01$ & $1.26691 \mathrm{e}+05$ & $4.94682 \mathrm{e}+02$ \\
\hline Worst & $6.23980 \mathrm{e}+10$ & $2.15599 \mathrm{e}+01$ & $8.25268 \mathrm{e}+01$ & $3.05519 \mathrm{e}+03$ & $3.75458 \mathrm{e}+01$ & $1.25816 \mathrm{e}+05$ & $6.46487 \mathrm{e}+02$ \\
\hline std & $1.15359 \mathrm{e}+10$ & $1.46944 \mathrm{e}-01$ & $4.83272 \mathrm{e}+00$ & $2.06318 \mathrm{e}+02$ & $4.91052 \mathrm{e}+00$ & $1.78593 \mathrm{e}+04$ & $5.21990 \mathrm{e}+01$ \\
\hline SR & 0 & 0 & 0 & 0 & 0 & 0 & 0 \\
\hline$\overline{v i o}$ & $3.82006 \mathrm{e}+04$ & $1.85570 \mathrm{e}+05$ & $5.70819 \mathrm{e}+04$ & $4.89022 \mathrm{e}+04$ & $5.63358 \mathrm{e}+04$ & $1.09278 \mathrm{e}+09$ & $7.34291 \mathrm{e}+04$ \\
\hline
\end{tabular}


Table 15: Function Values of HECO-PDE Achieved for 10D $($ MaxFES = 20000 $\times$ D $)$

\begin{tabular}{|c|c|c|c|c|c|c|c|}
\hline problem & C01 & $\mathrm{C} 02$ & $\mathrm{C03}$ & $\mathrm{C04}$ & $\mathrm{C} 05$ & $\mathrm{C} 06$ & $\mathrm{C} 07$ \\
\hline best & $0.00000 \mathrm{e}+00$ & $0.00000 \mathrm{e}+00$ & $0.00000 \mathrm{e}+00$ & $0.00000 \mathrm{e}+00$ & $0.00000 \mathrm{e}+00$ & $0.00000 \mathrm{e}+00$ & $-2.58748 \mathrm{e}+02$ \\
\hline median & $0.00000 \mathrm{e}+00$ & $0.00000 \mathrm{e}+00$ & $0.00000 \mathrm{e}+00$ & $0.00000 \mathrm{e}+00$ & $0.00000 \mathrm{e}+00$ & $0.00000 \mathrm{e}+00$ & $-1.17310 \mathrm{e}+03$ \\
\hline $\mathrm{c}$ & $\begin{array}{lll}0 & 0 & 0\end{array}$ & $\begin{array}{lll}0 & 0 & 0\end{array}$ & $\begin{array}{lll}0 & 0 & 0\end{array}$ & 000 & $\begin{array}{lll}0 & 0 & 0\end{array}$ & $\begin{array}{lll}0 & 0 & 0\end{array}$ & 002 \\
\hline $\bar{v}$ & $0.00000 \mathrm{e}+00$ & $0.00000 \mathrm{e}+00$ & $0.00000 \mathrm{e}+00$ & $0.00000 \mathrm{e}+00$ & $0.00000 \mathrm{e}+00$ & $0.00000 \mathrm{e}+00$ & $1.91686 \mathrm{e}-03$ \\
\hline Mean & $0.00000 \mathrm{e}+00$ & $0.00000 \mathrm{e}+00$ & $0.00000 \mathrm{e}+00$ & $0.00000 \mathrm{e}+00$ & $0.00000 \mathrm{e}+00$ & $0.00000 \mathrm{e}+00$ & $-7.46594 \mathrm{e}+02$ \\
\hline Worst & $0.00000 \mathrm{e}+00$ & $0.00000 \mathrm{e}+00$ & $0.00000 \mathrm{e}+00$ & $0.00000 \mathrm{e}+00$ & $0.00000 \mathrm{e}+00$ & $0.00000 \mathrm{e}+00$ & $-4.48664 e+02$ \\
\hline std & $0.00000 \mathrm{e}+00$ & $0.00000 \mathrm{e}+00$ & $0.00000 \mathrm{e}+00$ & $0.00000 \mathrm{e}+00$ & $0.00000 \mathrm{e}+00$ & $0.00000 \mathrm{e}+00$ & $7.86557 \mathrm{e}+02$ \\
\hline SR & 100 & 100 & 100 & 100 & 100 & 100 & 4 \\
\hline$\overline{v i o}$ & $0.00000 \mathrm{e}+00$ & $0.00000 \mathrm{e}+00$ & $0.00000 \mathrm{e}+00$ & $0.00000 \mathrm{e}+00$ & $0.00000 \mathrm{e}+00$ & $0.00000 \mathrm{e}+00$ & $2.31995 \mathrm{e}-03$ \\
\hline problem & $\mathrm{C} 08$ & C09 & C10 & C11 & C12 & C13 & C14 \\
\hline best & $-1.34840 \mathrm{e}-03$ & $-4.97525 \mathrm{e}-03$ & $-5.09647 \mathrm{e}-04$ & $-1.68819 \mathrm{e}-01$ & $3.98790 \mathrm{e}+00$ & $0.00000 \mathrm{e}+00$ & $2.37633 \mathrm{e}+00$ \\
\hline median & $-1.34840 \mathrm{e}-03$ & $-4.97525 \mathrm{e}-03$ & $-5.09647 e-04$ & $-1.68792 \mathrm{e}-01$ & $3.98883 \mathrm{e}+00$ & $0.00000 \mathrm{e}+00$ & $2.37633 \mathrm{e}+00$ \\
\hline $\mathrm{c}$ & $\begin{array}{lll}0 & 0 & 0\end{array}$ & $\begin{array}{lll}0 & 0 & 0\end{array}$ & $\begin{array}{lll}0 & 0 & 0\end{array}$ & $\begin{array}{lll}0 & 0 & 0\end{array}$ & $\begin{array}{lll}0 & 0 & 0\end{array}$ & $\begin{array}{lll}0 & 0 & 0\end{array}$ & $\begin{array}{lll}0 & 0 & 0\end{array}$ \\
\hline $\bar{v}$ & $0.00000 \mathrm{e}+00$ & $0.00000 \mathrm{e}+00$ & $0.00000 \mathrm{e}+00$ & $0.00000 \mathrm{e}+00$ & $0.00000 \mathrm{e}+00$ & $0.00000 \mathrm{e}+00$ & $0.00000 \mathrm{e}+00$ \\
\hline Mean & $-1.34840 \mathrm{e}-03$ & $-4.97525 \mathrm{e}-03$ & $-5.09647 \mathrm{e}-04$ & $-1.68092 \mathrm{e}-01$ & $3.98918 \mathrm{e}+00$ & $0.00000 \mathrm{e}+00$ & $2.37633 \mathrm{e}+00$ \\
\hline Worst & $-1.34840 \mathrm{e}-03$ & $-4.97525 \mathrm{e}-03$ & $-5.09647 \mathrm{e}-04$ & $-1.58782 \mathrm{e}-01$ & $3.99096 \mathrm{e}+00$ & $0.00000 \mathrm{e}+00$ & $2.37633 \mathrm{e}+00$ \\
\hline std & $3.48093 \mathrm{e}-16$ & $0.00000 \mathrm{e}+00$ & $3.31451 \mathrm{e}-15$ & $2.15091 \mathrm{e}-03$ & $1.08240 \mathrm{e}-03$ & $0.00000 \mathrm{e}+00$ & $0.00000 \mathrm{e}+00$ \\
\hline SR & 100 & 100 & 100 & 100 & 100 & 100 & 100 \\
\hline$\overline{v i o}$ & $0.00000 \mathrm{e}+00$ & $0.00000 \mathrm{e}+00$ & $0.00000 \mathrm{e}+00$ & $0.00000 \mathrm{e}+00$ & $0.00000 \mathrm{e}+00$ & $0.00000 \mathrm{e}+00$ & $0.00000 \mathrm{e}+00$ \\
\hline problem & C15 & C16 & $\mathrm{C} 17$ & C18 & C19 & $\mathrm{C} 20$ & $\mathrm{C} 21$ \\
\hline best & $2.35612 \mathrm{e}+00$ & $0.00000 \mathrm{e}+00$ & $1.08553 \mathrm{e}-02$ & $1.00000 \mathrm{e}+01$ & $0.00000 \mathrm{e}+00$ & $1.04005 \mathrm{e}-01$ & $3.98796 \mathrm{e}+00$ \\
\hline median & $2.35612 \mathrm{e}+00$ & $0.00000 \mathrm{e}+00$ & $1.08553 \mathrm{e}-02$ & $5.04203 \mathrm{e}+01$ & $0.00000 \mathrm{e}+00$ & $3.68418 \mathrm{e}-01$ & $3.98991 \mathrm{e}+00$ \\
\hline c & $\begin{array}{lll}0 & 0 & 0\end{array}$ & $\begin{array}{lll}0 & 0 & 0\end{array}$ & 100 & $\begin{array}{lll}0 & 0 & 0\end{array}$ & 100 & $\begin{array}{lll}0 & 0 & 0\end{array}$ & $\begin{array}{lll}0 & 0 & 0\end{array}$ \\
\hline $\bar{v}$ & $0.00000 \mathrm{e}+00$ & $0.00000 \mathrm{e}+00$ & $4.50000 \mathrm{e}+00$ & $0.00000 \mathrm{e}+00$ & $6.63359 \mathrm{e}+03$ & $0.00000 \mathrm{e}+00$ & $0.00000 \mathrm{e}+00$ \\
\hline Mean & $2.35612 \mathrm{e}+00$ & $0.00000 \mathrm{e}+00$ & $1.08553 \mathrm{e}-02$ & $3.91026 \mathrm{e}+01$ & $0.00000 \mathrm{e}+00$ & $3.58433 \mathrm{e}-01$ & $3.98967 \mathrm{e}+00$ \\
\hline Worst & $2.35612 \mathrm{e}+00$ & $0.00000 \mathrm{e}+00$ & $1.08553 \mathrm{e}-02$ & $5.04203 \mathrm{e}+01$ & $0.00000 \mathrm{e}+00$ & $5.20013 \mathrm{e}-01$ & $3.99246 \mathrm{e}+00$ \\
\hline std & $1.11995 \mathrm{e}-15$ & $0.00000 \mathrm{e}+00$ & $1.08780 \mathrm{e}-16$ & $1.81487 \mathrm{e}+01$ & $0.00000 \mathrm{e}+00$ & $1.07653 \mathrm{e}-01$ & $1.26111 \mathrm{e}-03$ \\
\hline SR & 100 & 100 & 0 & 100 & 0 & 100 & 100 \\
\hline vio & $0.00000 \mathrm{e}+00$ & $0.00000 \mathrm{e}+00$ & $4.50000 \mathrm{e}+00$ & $0.00000 \mathrm{e}+00$ & $6.63359 \mathrm{e}+03$ & $0.00000 \mathrm{e}+00$ & $0.00000 \mathrm{e}+00$ \\
\hline problem & $\mathrm{C} 22$ & $\mathrm{C} 23$ & $\mathrm{C} 24$ & $\mathrm{C} 25$ & $\mathrm{C} 26$ & $\mathrm{C} 27$ & $\mathrm{C} 28$ \\
\hline best & $3.48642 \mathrm{e}-27$ & $2.37633 \mathrm{e}+00$ & $2.35612 \mathrm{e}+00$ & $0.00000 \mathrm{e}+00$ & $1.08553 \mathrm{e}-02$ & $9.05515 \mathrm{e}+01$ & $0.00000 \mathrm{e}+00$ \\
\hline median & $3.48642 \mathrm{e}-27$ & $2.37633 \mathrm{e}+00$ & $2.35612 \mathrm{e}+00$ & $0.00000 \mathrm{e}+00$ & $1.08553 \mathrm{e}-02$ & $9.57215 \mathrm{e}+01$ & $0.00000 \mathrm{e}+00$ \\
\hline c & $\begin{array}{lll}0 & 0 & 0\end{array}$ & $\begin{array}{lll}0 & 0 & 0\end{array}$ & $\begin{array}{lll}0 & 0 & 0\end{array}$ & $\begin{array}{lll}0 & 0 & 0\end{array}$ & 100 & $\begin{array}{lll}0 & 0 & 0\end{array}$ & 100 \\
\hline $\bar{v}$ & $0.00000 \mathrm{e}+00$ & $0.00000 \mathrm{e}+00$ & $0.00000 \mathrm{e}+00$ & $0.00000 \mathrm{e}+00$ & $4.50000 \mathrm{e}+00$ & $0.00000 \mathrm{e}+00$ & $6.63359 \mathrm{e}+03$ \\
\hline Mean & $3.49026 \mathrm{e}-27$ & $2.37633 \mathrm{e}+00$ & $2.35612 \mathrm{e}+00$ & $0.00000 \mathrm{e}+00$ & $7.37529 \mathrm{e}-02$ & $9.40671 \mathrm{e}+01$ & $0.00000 \mathrm{e}+00$ \\
\hline Worst & $3.51844 \mathrm{e}-27$ & $2.37633 \mathrm{e}+00$ & $2.35612 \mathrm{e}+00$ & $0.00000 \mathrm{e}+00$ & $7.10694 \mathrm{e}-01$ & $9.57217 \mathrm{e}+01$ & $0.00000 \mathrm{e}+00$ \\
\hline std & $1.04062 \mathrm{e}-29$ & $5.21413 \mathrm{e}-07$ & $5.18534 \mathrm{e}-08$ & $0.00000 \mathrm{e}+00$ & $1.75700 \mathrm{e}-01$ & $2.41167 \mathrm{e}+00$ & $0.00000 \mathrm{e}+00$ \\
\hline SR & 100 & 100 & 100 & 100 & 0 & 100 & 0 \\
\hline$\overline{v i o}$ & $0.00000 \mathrm{e}+00$ & $0.00000 \mathrm{e}+00$ & $0.00000 \mathrm{e}+00$ & $0.00000 \mathrm{e}+00$ & $4.50000 \mathrm{e}+00$ & $0.00000 \mathrm{e}+00$ & $6.63359 \mathrm{e}+03$ \\
\hline
\end{tabular}


Table 16: Function Values of HECO-PDE Achieved for 30D $($ MaxFES $=20000 \times D)$

\begin{tabular}{|c|c|c|c|c|c|c|c|}
\hline problem & $\mathrm{C} 01$ & $\mathrm{C} 02$ & $\mathrm{C} 03$ & $\mathrm{C} 04$ & $\mathrm{C} 05$ & C06 & $\mathrm{C} 07$ \\
\hline best & $0.00000 \mathrm{e}+00$ & $0.00000 \mathrm{e}+00$ & $0.00000 \mathrm{e}+00$ & $1.35728 \mathrm{e}+01$ & $0.00000 \mathrm{e}+00$ & $0.00000 \mathrm{e}+00$ & $-2.45791 \mathrm{e}+03$ \\
\hline median & $9.33075 \mathrm{e}-30$ & $1.17959 \mathrm{e}-29$ & $3.49071 \mathrm{e}-29$ & $1.35728 \mathrm{e}+01$ & $0.00000 \mathrm{e}+00$ & $0.00000 \mathrm{e}+00$ & $-2.68829 \mathrm{e}+03$ \\
\hline $\mathrm{c}$ & 000 & 000 & 000 & 000 & 000 & 000 & 002 \\
\hline $\bar{v}$ & $0.00000 \mathrm{e}+00$ & $0.00000 \mathrm{e}+00$ & $0.00000 \mathrm{e}+00$ & $0.00000 \mathrm{e}+00$ & $0.00000 \mathrm{e}+00$ & $0.00000 \mathrm{e}+00$ & $7.93332 \mathrm{e}-04$ \\
\hline Mean & $1.59799 \mathrm{e}-29$ & $1.96421 \mathrm{e}-29$ & $3.95614 \mathrm{e}-29$ & $1.35728 \mathrm{e}+01$ & $0.00000 \mathrm{e}+00$ & $0.00000 \mathrm{e}+00$ & $-1.94723 e+03$ \\
\hline Worst & $7.51020 \mathrm{e}-29$ & $6.70162 \mathrm{e}-29$ & $1.34895 \mathrm{e}-28$ & $1.35728 \mathrm{e}+01$ & $0.00000 \mathrm{e}+00$ & $0.00000 \mathrm{e}+00$ & $-2.51299 \mathrm{e}+03$ \\
\hline std & $2.11761 \mathrm{e}-29$ & $1.97404 \mathrm{e}-29$ & $3.35121 \mathrm{e}-29$ & $1.28144 \mathrm{e}-14$ & $0.00000 \mathrm{e}+00$ & $0.00000 \mathrm{e}+00$ & $9.19644 \mathrm{e}+02$ \\
\hline SR & 100 & 100 & 100 & 100 & 100 & 100 & 0 \\
\hline$\overline{v i o}$ & $0.00000 \mathrm{e}+00$ & $0.00000 \mathrm{e}+00$ & $0.00000 \mathrm{e}+00$ & $0.00000 \mathrm{e}+00$ & $0.00000 \mathrm{e}+00$ & $0.00000 \mathrm{e}+00$ & $1.05592 \mathrm{e}-03$ \\
\hline problem & $\mathrm{C} 08$ & C09 & C10 & C11 & C12 & C13 & C14 \\
\hline best & $-2.83981 \mathrm{e}-04$ & $-2.66551 \mathrm{e}-03$ & $-1.02842 \mathrm{e}-04$ & $-9.24713 \mathrm{e}-01$ & $3.98253 \mathrm{e}+00$ & $0.00000 \mathrm{e}+00$ & $1.40852 \mathrm{e}+00$ \\
\hline median & $-2.83981 \mathrm{e}-04$ & $-2.66551 \mathrm{e}-03$ & $-1.02842 \mathrm{e}-04$ & $-8.77334 \mathrm{e}-01$ & $3.98257 \mathrm{e}+00$ & $0.00000 \mathrm{e}+00$ & $1.40852 \mathrm{e}+00$ \\
\hline c & $\begin{array}{lll}0 & 0 & 0\end{array}$ & $\begin{array}{lll}0 & 0 & 0\end{array}$ & $\begin{array}{lll}0 & 0 & 0\end{array}$ & $\begin{array}{lll}0 & 0 & 0\end{array}$ & $\begin{array}{lll}0 & 0 & 0\end{array}$ & $\begin{array}{lll}0 & 0 & 0\end{array}$ & $\begin{array}{lll}0 & 0 & 0\end{array}$ \\
\hline $\bar{v}$ & $0.00000 \mathrm{e}+00$ & $0.00000 \mathrm{e}+00$ & $0.00000 \mathrm{e}+00$ & $0.00000 \mathrm{e}+00$ & $0.00000 \mathrm{e}+00$ & $0.00000 \mathrm{e}+00$ & $0.00000 \mathrm{e}+00$ \\
\hline Mean & $-2.83981 \mathrm{e}-04$ & $-2.66551 \mathrm{e}-03$ & $-1.02842 \mathrm{e}-04$ & $-1.87293 e+02$ & $3.98269 \mathrm{e}+00$ & $0.00000 \mathrm{e}+00$ & $1.40852 \mathrm{e}+00$ \\
\hline Worst & $-2.83981 \mathrm{e}-04$ & $-2.66551 \mathrm{e}-03$ & $-1.02840 \mathrm{e}-04$ & $-1.25684 \mathrm{e}+03$ & $3.98341 \mathrm{e}+00$ & $0.00000 \mathrm{e}+00$ & $1.40852 \mathrm{e}+00$ \\
\hline std & $5.00769 \mathrm{e}-11$ & $3.79326 \mathrm{e}-16$ & $2.41501 \mathrm{e}-10$ & $3.71804 \mathrm{e}+02$ & $2.14922 \mathrm{e}-04$ & $0.00000 \mathrm{e}+00$ & $7.00761 \mathrm{e}-16$ \\
\hline $\mathrm{SR}$ & 100 & 100 & 100 & 64 & 100 & 100 & 100 \\
\hline$\overline{\text { vio }}$ & $0.00000 \mathrm{e}+00$ & $0.00000 \mathrm{e}+00$ & $0.00000 \mathrm{e}+00$ & $1.26005 \mathrm{e}+01$ & $0.00000 \mathrm{e}+00$ & $0.00000 \mathrm{e}+00$ & $0.00000 \mathrm{e}+00$ \\
\hline problem & C15 & C16 & C17 & C18 & C19 & $\mathrm{C} 20$ & C21 \\
\hline best & $2.35612 \mathrm{e}+00$ & $0.00000 \mathrm{e}+00$ & $3.08555 \mathrm{e}-02$ & $3.00000 \mathrm{e}+01$ & $0.00000 \mathrm{e}+00$ & $2.50134 \mathrm{e}+00$ & $3.98253 \mathrm{e}+00$ \\
\hline median & $2.35612 \mathrm{e}+00$ & $0.00000 \mathrm{e}+00$ & $1.44943 \mathrm{e}+00$ & $5.34725 \mathrm{e}+01$ & $0.00000 \mathrm{e}+00$ & $2.84742 \mathrm{e}+00$ & $3.98262 \mathrm{e}+00$ \\
\hline $\mathrm{c}$ & $\begin{array}{lll}0 & 0 & 0\end{array}$ & $\begin{array}{lll}0 & 0 & 0\end{array}$ & 100 & $\begin{array}{lll}0 & 0 & 0\end{array}$ & 100 & $\begin{array}{lll}0 & 0 & 0\end{array}$ & $\begin{array}{lll}0 & 0 & 0\end{array}$ \\
\hline $\bar{v}$ & $0.00000 \mathrm{e}+00$ & $0.00000 \mathrm{e}+00$ & $1.45000 \mathrm{e}+01$ & $0.00000 \mathrm{e}+00$ & $2.13749 \mathrm{e}+04$ & $0.00000 \mathrm{e}+00$ & $0.00000 \mathrm{e}+00$ \\
\hline Mean & $2.35612 \mathrm{e}+00$ & $0.00000 \mathrm{e}+00$ & $6.51135 \mathrm{e}-01$ & $4.85514 \mathrm{e}+01$ & $0.00000 \mathrm{e}+00$ & $2.87611 \mathrm{e}+00$ & $3.98269 \mathrm{e}+00$ \\
\hline Worst & $2.35612 \mathrm{e}+00$ & $0.00000 \mathrm{e}+00$ & $9.51999 \mathrm{e}-01$ & $5.82173 \mathrm{e}+01$ & $0.00000 \mathrm{e}+00$ & $3.19426 \mathrm{e}+00$ & $3.98327 \mathrm{e}+00$ \\
\hline std & $1.13742 \mathrm{e}-15$ & $0.00000 \mathrm{e}+00$ & $4.82474 \mathrm{e}-01$ & $8.31285 \mathrm{e}+00$ & $0.00000 \mathrm{e}+00$ & $1.88661 \mathrm{e}-01$ & $1.94653 \mathrm{e}-04$ \\
\hline SR & 100 & 100 & 0 & 100 & 0 & 100 & 100 \\
\hline$\overline{v i o}$ & $0.00000 \mathrm{e}+00$ & $0.00000 \mathrm{e}+00$ & $1.49400 \mathrm{e}+01$ & $0.00000 \mathrm{e}+00$ & $2.13749 \mathrm{e}+04$ & $0.00000 \mathrm{e}+00$ & $0.00000 \mathrm{e}+00$ \\
\hline problem & $\mathrm{C} 22$ & $\mathrm{C} 23$ & $\mathrm{C} 24$ & $\mathrm{C} 25$ & $\mathrm{C} 26$ & C27 & C28 \\
\hline best & $3.59077 \mathrm{e}-14$ & $1.40852 \mathrm{e}+00$ & $2.35612 \mathrm{e}+00$ & $0.00000 \mathrm{e}+00$ & $6.11945 \mathrm{e}-01$ & $2.08760 \mathrm{e}+02$ & $0.00000 \mathrm{e}+00$ \\
\hline median & $7.91069 \mathrm{e}-10$ & $1.40852 \mathrm{e}+00$ & $2.35612 \mathrm{e}+00$ & $0.00000 \mathrm{e}+00$ & $8.53762 \mathrm{e}-01$ & $2.51342 \mathrm{e}+02$ & $3.33028 \mathrm{e}-06$ \\
\hline $\mathrm{c}$ & $\begin{array}{lll}0 & 0 & 0\end{array}$ & $\begin{array}{lll}0 & 0 & 0\end{array}$ & $\begin{array}{lll}0 & 0 & 0\end{array}$ & $\begin{array}{lll}0 & 0 & 0\end{array}$ & 100 & 000 & 100 \\
\hline $\bar{v}$ & $0.00000 \mathrm{e}+00$ & $0.00000 \mathrm{e}+00$ & $0.00000 \mathrm{e}+00$ & $0.00000 \mathrm{e}+00$ & $1.55000 \mathrm{e}+01$ & $0.00000 \mathrm{e}+00$ & $2.13749 \mathrm{e}+04$ \\
\hline Mean & $4.13459 \mathrm{e}-08$ & $1.40853 \mathrm{e}+00$ & $2.35612 \mathrm{e}+00$ & $0.00000 \mathrm{e}+00$ & $8.41942 \mathrm{e}-01$ & $2.36013 \mathrm{e}+02$ & $4.23646 \mathrm{e}-06$ \\
\hline Worst & $7.52530 \mathrm{e}-07$ & $1.40858 \mathrm{e}+00$ & $2.35612 \mathrm{e}+00$ & $0.00000 \mathrm{e}+00$ & $1.01187 \mathrm{e}+00$ & $2.51347 \mathrm{e}+02$ & $1.77740 \mathrm{e}-05$ \\
\hline std & $1.48613 \mathrm{e}-07$ & $1.46320 \mathrm{e}-05$ & $8.05949 \mathrm{e}-08$ & $0.00000 \mathrm{e}+00$ & $8.41683 \mathrm{e}-02$ & $2.04397 \mathrm{e}+01$ & $3.62229 \mathrm{e}-06$ \\
\hline SR & 100 & 100 & 100 & 100 & 0 & 100 & 0 \\
\hline$\overline{\text { vio }}$ & $0.00000 \mathrm{e}+00$ & $0.00000 \mathrm{e}+00$ & $0.00000 \mathrm{e}+00$ & $0.00000 \mathrm{e}+00$ & $1.55000 \mathrm{e}+01$ & $0.00000 \mathrm{e}+00$ & $2.13749 \mathrm{e}+04$ \\
\hline
\end{tabular}


Table 17: Function Values of HECO-PDE Achieved for 50D $($ MaxFES = 20000 $\times$ D)

\begin{tabular}{|c|c|c|c|c|c|c|c|}
\hline problem & $\mathrm{C} 01$ & $\mathrm{C} 02$ & $\mathrm{C} 03$ & $\mathrm{C} 04$ & C05 & C06 & C07 \\
\hline best & $1.04672 \mathrm{e}-28$ & $4.57539 \mathrm{e}-29$ & $1.81832 \mathrm{e}-28$ & $1.35728 \mathrm{e}+01$ & $0.00000 \mathrm{e}+00$ & $0.00000 \mathrm{e}+00$ & $-3.78512 \mathrm{e}+03$ \\
\hline median & $2.08259 \mathrm{e}-28$ & $2.17442 \mathrm{e}-28$ & $3.30138 \mathrm{e}-28$ & $1.35728 \mathrm{e}+01$ & $9.12120 \mathrm{e}-30$ & $1.41843 \mathrm{e}+02$ & $-8.31534 \mathrm{e}+02$ \\
\hline $\mathrm{c}$ & $\begin{array}{lll}0 & 0 & 0\end{array}$ & $\begin{array}{lllllllll}0 & 0 & 0\end{array}$ & $\begin{array}{lll}0 & 0 & 0\end{array}$ & 000 & 000 & $\begin{array}{lll}0 & 0 & 0\end{array}$ & 002 \\
\hline $\bar{v}$ & $0.00000 \mathrm{e}+00$ & $0.00000 \mathrm{e}+00$ & $0.00000 \mathrm{e}+00$ & $0.00000 \mathrm{e}+00$ & $0.00000 \mathrm{e}+00$ & $0.00000 \mathrm{e}+00$ & $5.66334 \mathrm{e}-04$ \\
\hline Mean & $2.51258 \mathrm{e}-28$ & $2.17115 \mathrm{e}-28$ & $4.12290 \mathrm{e}-28$ & $1.35728 \mathrm{e}+01$ & $4.52846 \mathrm{e}-29$ & $1.16974 \mathrm{e}+02$ & $-2.79070 \mathrm{e}+03$ \\
\hline Worst & $5.16815 \mathrm{e}-28$ & $4.33787 \mathrm{e}-28$ & $8.70114 \mathrm{e}-28$ & $1.35728 \mathrm{e}+01$ & $4.25245 \mathrm{e}-28$ & $1.66179 \mathrm{e}+02$ & $-3.52267 \mathrm{e}+03$ \\
\hline std & $1.21265 \mathrm{e}-28$ & $8.04570 \mathrm{e}-29$ & $1.98135 \mathrm{e}-28$ & $2.10181 \mathrm{e}-15$ & $8.97841 \mathrm{e}-29$ & $9.83836 \mathrm{e}+01$ & $9.79449 \mathrm{e}+02$ \\
\hline SR & 100 & 100 & 100 & 100 & 100 & 76 & 12 \\
\hline$\overline{v i o}$ & $0.00000 \mathrm{e}+00$ & $0.00000 \mathrm{e}+00$ & $0.00000 \mathrm{e}+00$ & $0.00000 \mathrm{e}+00$ & $0.00000 \mathrm{e}+00$ & $2.42773 \mathrm{e}-04$ & $8.24621 \mathrm{e}-04$ \\
\hline problem & $\mathrm{C} 08$ & C09 & $\mathrm{C} 10$ & C11 & C12 & C13 & C14 \\
\hline best & $-1.34466 \mathrm{e}-04$ & $-2.03709 \mathrm{e}-03$ & $-4.82659 \mathrm{e}-05$ & $-3.11531 e+02$ & $3.98145 \mathrm{e}+00$ & $0.00000 \mathrm{e}+00$ & $1.09995 \mathrm{e}+00$ \\
\hline median & $-1.33456 \mathrm{e}-04$ & $-2.03709 \mathrm{e}-03$ & $-4.82628 \mathrm{e}-05$ & $-1.62884 \mathrm{e}+03$ & $3.98150 \mathrm{e}+00$ & $1.33101 \mathrm{e}-27$ & $1.09995 \mathrm{e}+00$ \\
\hline c & $\begin{array}{lll}0 & 0 & 0\end{array}$ & $\begin{array}{lll}0 & 0 & 0\end{array}$ & $\begin{array}{lll}0 & 0 & 0\end{array}$ & 100 & $\begin{array}{lll}0 & 0 & 0\end{array}$ & $\begin{array}{lll}0 & 0 & 0\end{array}$ & $\begin{array}{lll}0 & 0 & 0\end{array}$ \\
\hline $\bar{v}$ & $0.00000 \mathrm{e}+00$ & $0.00000 \mathrm{e}+00$ & $0.00000 \mathrm{e}+00$ & $3.29899 \mathrm{e}+01$ & $0.00000 \mathrm{e}+00$ & $0.00000 \mathrm{e}+00$ & $0.00000 \mathrm{e}+00$ \\
\hline Mean & $-1.31402 \mathrm{e}-04$ & $-2.03709 \mathrm{e}-03$ & $-4.82606 \mathrm{e}-05$ & $-1.54196 \mathrm{e}+03$ & $4.22857 \mathrm{e}+00$ & $1.59465 \mathrm{e}-01$ & $1.10073 \mathrm{e}+00$ \\
\hline Worst & $-1.12487 \mathrm{e}-04$ & $-2.03709 \mathrm{e}-03$ & $-4.82487 \mathrm{e}-05$ & $-2.67003 e+03$ & $7.06997 \mathrm{e}+00$ & $3.98662 \mathrm{e}+00$ & $1.11946 \mathrm{e}+00$ \\
\hline std & $4.80757 \mathrm{e}-06$ & $1.40141 \mathrm{e}-15$ & $4.96661 \mathrm{e}-09$ & $6.80331 \mathrm{e}+02$ & $8.37725 \mathrm{e}-01$ & $7.81216 \mathrm{e}-01$ & $3.82241 \mathrm{e}-03$ \\
\hline $\mathrm{SR}$ & 100 & 100 & 100 & 0 & 100 & 100 & 100 \\
\hline$\overline{v i o}$ & $0.00000 \mathrm{e}+00$ & $0.00000 \mathrm{e}+00$ & $0.00000 \mathrm{e}+00$ & $3.55113 \mathrm{e}+01$ & $0.00000 \mathrm{e}+00$ & $0.00000 \mathrm{e}+00$ & $0.00000 \mathrm{e}+00$ \\
\hline problem & $\mathrm{C} 15$ & C16 & $\mathrm{C} 17$ & $\mathrm{C} 18$ & C19 & $\mathrm{C} 20$ & C21 \\
\hline best & $2.35612 \mathrm{e}+00$ & $0.00000 \mathrm{e}+00$ & $6.04826 \mathrm{e}-02$ & $4.42174 \mathrm{e}+01$ & $0.00000 \mathrm{e}+00$ & $5.15997 \mathrm{e}+00$ & $3.98145 \mathrm{e}+00$ \\
\hline median & $2.35612 \mathrm{e}+00$ & $0.00000 \mathrm{e}+00$ & $5.45231 \mathrm{e}-01$ & $4.61632 \mathrm{e}+01$ & $0.00000 \mathrm{e}+00$ & $6.24310 \mathrm{e}+00$ & $3.98149 \mathrm{e}+00$ \\
\hline $\mathrm{c}$ & $\begin{array}{lll}0 & 0 & 0\end{array}$ & $\begin{array}{lll}0 & 0 & 0\end{array}$ & 100 & $\begin{array}{lll}0 & 0 & 0\end{array}$ & 100 & $\begin{array}{lll}0 & 0 & 0\end{array}$ & $\begin{array}{lll}0 & 0 & 0\end{array}$ \\
\hline $\bar{v}$ & $0.00000 \mathrm{e}+00$ & $0.00000 \mathrm{e}+00$ & $2.55000 \mathrm{e}+01$ & $0.00000 \mathrm{e}+00$ & $3.61162 \mathrm{e}+04$ & $0.00000 \mathrm{e}+00$ & $0.00000 \mathrm{e}+00$ \\
\hline Mean & $2.35612 \mathrm{e}+00$ & $0.00000 \mathrm{e}+00$ & $6.17102 \mathrm{e}-01$ & $4.76107 \mathrm{e}+01$ & $0.00000 \mathrm{e}+00$ & $6.10145 \mathrm{e}+00$ & $4.10501 \mathrm{e}+00$ \\
\hline Worst & $2.35612 \mathrm{e}+00$ & $0.00000 \mathrm{e}+00$ & $9.20881 \mathrm{e}-01$ & $6.22173 \mathrm{e}+01$ & $0.00000 \mathrm{e}+00$ & $6.92786 \mathrm{e}+00$ & $7.06871 \mathrm{e}+00$ \\
\hline std & $1.55368 \mathrm{e}-15$ & $0.00000 \mathrm{e}+00$ & $3.32818 \mathrm{e}-01$ & $3.65297 \mathrm{e}+00$ & $0.00000 \mathrm{e}+00$ & $4.34170 \mathrm{e}-01$ & $6.04962 \mathrm{e}-01$ \\
\hline SR & 100 & 100 & 0 & 100 & 0 & 100 & 100 \\
\hline vio & $0.00000 \mathrm{e}+00$ & $0.00000 \mathrm{e}+00$ & $2.52200 \mathrm{e}+01$ & $0.00000 \mathrm{e}+00$ & $3.61162 \mathrm{e}+04$ & $0.00000 \mathrm{e}+00$ & $0.00000 \mathrm{e}+00$ \\
\hline problem & $\mathrm{C} 22$ & $\mathrm{C} 23$ & $\mathrm{C} 24$ & $\mathrm{C} 25$ & $\mathrm{C} 26$ & C27 & C28 \\
\hline best & $5.13492 \mathrm{e}-01$ & $1.09995 \mathrm{e}+00$ & $2.35612 \mathrm{e}+00$ & $0.00000 \mathrm{e}+00$ & $8.01254 \mathrm{e}-01$ & $2.47635 \mathrm{e}+02$ & $1.65653 \mathrm{e}-05$ \\
\hline median & $1.40918 \mathrm{e}+01$ & $1.09996 \mathrm{e}+00$ & $2.35612 \mathrm{e}+00$ & $0.00000 \mathrm{e}+00$ & $9.36281 \mathrm{e}-01$ & $2.64205 \mathrm{e}+02$ & $3.28074 \mathrm{e}+00$ \\
\hline $\mathrm{c}$ & $\begin{array}{lll}0 & 0 & 0\end{array}$ & $\begin{array}{lll}0 & 0 & 0\end{array}$ & $\begin{array}{lll}0 & 0 & 0\end{array}$ & $\begin{array}{lll}0 & 0 & 0\end{array}$ & 100 & 000 & 100 \\
\hline $\bar{v}$ & $0.00000 \mathrm{e}+00$ & $0.00000 \mathrm{e}+00$ & $0.00000 \mathrm{e}+00$ & $0.00000 \mathrm{e}+00$ & $2.55000 \mathrm{e}+01$ & $0.00000 \mathrm{e}+00$ & $3.61248 \mathrm{e}+04$ \\
\hline Mean & $1.34654 \mathrm{e}+01$ & $1.09997 \mathrm{e}+00$ & $2.35612 \mathrm{e}+00$ & $3.02025 \mathrm{e}-14$ & $9.31995 \mathrm{e}-01$ & $2.58242 \mathrm{e}+02$ & $2.76288 \mathrm{e}+00$ \\
\hline Worst & $1.60992 \mathrm{e}+01$ & $1.10009 \mathrm{e}+00$ & $2.35612 \mathrm{e}+00$ & $7.55062 \mathrm{e}-13$ & $1.01406 \mathrm{e}+00$ & $2.64215 \mathrm{e}+02$ & $7.12744 \mathrm{e}+00$ \\
\hline std & $2.82924 \mathrm{e}+00$ & $3.13374 \mathrm{e}-05$ & $1.72250 \mathrm{e}-08$ & $1.47961 \mathrm{e}-13$ & $5.29458 \mathrm{e}-02$ & $7.95311 \mathrm{e}+00$ & $2.53458 \mathrm{e}+00$ \\
\hline SR & 100 & 100 & 100 & 100 & 0 & 100 & 0 \\
\hline$\overline{\text { vio }}$ & $0.00000 \mathrm{e}+00$ & $0.00000 \mathrm{e}+00$ & $0.00000 \mathrm{e}+00$ & $0.00000 \mathrm{e}+00$ & $2.55000 \mathrm{e}+01$ & $0.00000 \mathrm{e}+00$ & $3.61255 \mathrm{e}+04$ \\
\hline
\end{tabular}


Table 18: Function Values of HECO-PDE Achieved for 100D $(\operatorname{MaxFES=20000\times D)}$

\begin{tabular}{|c|c|c|c|c|c|c|c|}
\hline problem & $\mathrm{C} 01$ & $\mathrm{C} 02$ & $\mathrm{C} 03$ & $\mathrm{C} 04$ & $\mathrm{C05}$ & C06 & C07 \\
\hline best & $4.74860 \mathrm{e}-24$ & $2.08968 \mathrm{e}-23$ & $1.09781 \mathrm{e}-23$ & $1.35728 \mathrm{e}+01$ & $1.24693 \mathrm{e}-16$ & $5.85856 \mathrm{e}+02$ & $-6.04093 e+03$ \\
\hline median & $2.96855 \mathrm{e}-22$ & $7.65701 \mathrm{e}-22$ & $2.63175 \mathrm{e}-22$ & $1.35728 \mathrm{e}+01$ & $5.60537 \mathrm{e}-15$ & $9.10968 \mathrm{e}+02$ & $-5.12059 e+03$ \\
\hline $\mathrm{c}$ & $\begin{array}{lll}0 & 0 & 0\end{array}$ & $\begin{array}{lllllllll}0 & 0 & 0\end{array}$ & $\begin{array}{lll}0 & 0 & 0\end{array}$ & 000 & 000 & $\begin{array}{lll}0 & 0 & 0\end{array}$ & 002 \\
\hline $\bar{v}$ & $0.00000 \mathrm{e}+00$ & $0.00000 \mathrm{e}+00$ & $0.00000 \mathrm{e}+00$ & $0.00000 \mathrm{e}+00$ & $0.00000 \mathrm{e}+00$ & $0.00000 \mathrm{e}+00$ & $3.43542 \mathrm{e}-04$ \\
\hline Mean & $7.92774 \mathrm{e}-22$ & $6.12831 \mathrm{e}-21$ & $1.04964 \mathrm{e}-21$ & $1.36666 \mathrm{e}+01$ & $1.13652 \mathrm{e}-13$ & $8.17227 \mathrm{e}+02$ & $-4.19316 \mathrm{e}+03$ \\
\hline Worst & $4.80812 \mathrm{e}-21$ & $4.10046 \mathrm{e}-20$ & $7.98385 \mathrm{e}-21$ & $1.59192 \mathrm{e}+01$ & $1.63788 \mathrm{e}-12$ & $8.24776 \mathrm{e}+02$ & $-5.64890 e+03$ \\
\hline std & $1.10948 \mathrm{e}-21$ & $1.11876 \mathrm{e}-20$ & $1.84224 \mathrm{e}-21$ & $4.59810 \mathrm{e}-01$ & $3.33214 \mathrm{e}-13$ & $1.19466 \mathrm{e}+02$ & $1.20840 \mathrm{e}+03$ \\
\hline SR & 100 & 100 & 100 & 100 & 100 & 64 & 20 \\
\hline$\overline{v i o}$ & $0.00000 \mathrm{e}+00$ & $0.00000 \mathrm{e}+00$ & $0.00000 \mathrm{e}+00$ & $0.00000 \mathrm{e}+00$ & $0.00000 \mathrm{e}+00$ & $1.07363 \mathrm{e}-03$ & $4.02313 \mathrm{e}-04$ \\
\hline problem & $\mathrm{C} 08$ & C09 & $\mathrm{C} 10$ & C11 & C12 & C13 & C14 \\
\hline best & $9.89426 \mathrm{e}-05$ & $0.00000 \mathrm{e}+00$ & $-1.69783 \mathrm{e}-05$ & $-6.28804 e+03$ & $1.88621 \mathrm{e}+01$ & $3.32042 \mathrm{e}+01$ & $7.84202 \mathrm{e}-01$ \\
\hline median & $4.62686 \mathrm{e}-04$ & $0.00000 \mathrm{e}+00$ & $-1.66940 \mathrm{e}-05$ & $-6.98033 e+03$ & $3.16345 \mathrm{e}+01$ & $3.32046 \mathrm{e}+01$ & $7.86865 \mathrm{e}-01$ \\
\hline c & $\begin{array}{lll}0 & 0 & 0\end{array}$ & $\begin{array}{lll}0 & 0 & 0\end{array}$ & $\begin{array}{lll}0 & 0 & 0\end{array}$ & 100 & $\begin{array}{lll}0 & 0 & 0\end{array}$ & $\begin{array}{lll}0 & 0 & 0\end{array}$ & $\begin{array}{lll}0 & 0 & 0\end{array}$ \\
\hline $\bar{v}$ & $0.00000 \mathrm{e}+00$ & $0.00000 \mathrm{e}+00$ & $0.00000 \mathrm{e}+00$ & $8.12562 \mathrm{e}+01$ & $0.00000 \mathrm{e}+00$ & $0.00000 \mathrm{e}+00$ & $0.00000 \mathrm{e}+00$ \\
\hline Mean & $4.54336 \mathrm{e}-04$ & $0.00000 \mathrm{e}+00$ & $-1.67005 \mathrm{e}-05$ & $-6.83288 \mathrm{e}+03$ & $3.11306 \mathrm{e}+01$ & $3.36826 \mathrm{e}+01$ & $7.96313 \mathrm{e}-01$ \\
\hline Worst & $9.65755 \mathrm{e}-04$ & $0.00000 \mathrm{e}+00$ & $-1.62289 \mathrm{e}-05$ & $-6.23668 \mathrm{e}+03$ & $3.18122 \mathrm{e}+01$ & $3.71880 \mathrm{e}+01$ & $8.38008 \mathrm{e}-01$ \\
\hline std & $1.64588 \mathrm{e}-04$ & $0.00000 \mathrm{e}+00$ & $2.14329 \mathrm{e}-07$ & $3.27748 \mathrm{e}+02$ & $2.50488 \mathrm{e}+00$ & $1.29435 \mathrm{e}+00$ & $1.52042 \mathrm{e}-02$ \\
\hline $\mathrm{SR}$ & 100 & 100 & 100 & 0 & 100 & 100 & 100 \\
\hline$\overline{v i o}$ & $0.00000 \mathrm{e}+00$ & $0.00000 \mathrm{e}+00$ & $0.00000 \mathrm{e}+00$ & $9.43117 \mathrm{e}+01$ & $0.00000 \mathrm{e}+00$ & $0.00000 \mathrm{e}+00$ & $0.00000 \mathrm{e}+00$ \\
\hline problem & C15 & C16 & $\mathrm{C} 17$ & C18 & C19 & $\mathrm{C} 20$ & $\mathrm{C} 21$ \\
\hline best & $2.35612 \mathrm{e}+00$ & $0.00000 \mathrm{e}+00$ & $2.59387 \mathrm{e}-01$ & $4.62791 \mathrm{e}+01$ & $0.00000 \mathrm{e}+00$ & $1.47876 \mathrm{e}+01$ & $1.20688 \mathrm{e}+01$ \\
\hline median & $2.35612 \mathrm{e}+00$ & $0.00000 \mathrm{e}+00$ & $8.79248 \mathrm{e}-01$ & $1.30626 \mathrm{e}+02$ & $0.00000 \mathrm{e}+00$ & $1.58000 \mathrm{e}+01$ & $3.15911 \mathrm{e}+01$ \\
\hline $\mathrm{c}$ & $\begin{array}{lll}0 & 0 & 0\end{array}$ & $\begin{array}{lll}0 & 0 & 0\end{array}$ & 100 & 100 & 100 & $\begin{array}{lll}0 & 0 & 0\end{array}$ & $\begin{array}{lll}0 & 0 & 0\end{array}$ \\
\hline $\bar{v}$ & $0.00000 \mathrm{e}+00$ & $0.00000 \mathrm{e}+00$ & $5.05000 \mathrm{e}+01$ & $2.11765 \mathrm{e}+01$ & $7.29695 \mathrm{e}+04$ & $0.00000 \mathrm{e}+00$ & $0.00000 \mathrm{e}+00$ \\
\hline Mean & $2.35612 \mathrm{e}+00$ & $0.00000 \mathrm{e}+00$ & $8.01474 \mathrm{e}-01$ & $8.51306 \mathrm{e}+01$ & $1.68587 \mathrm{e}-09$ & $1.58580 \mathrm{e}+01$ & $3.03467 \mathrm{e}+01$ \\
\hline Worst & $2.35612 \mathrm{e}+00$ & $0.00000 \mathrm{e}+00$ & $1.01669 \mathrm{e}+00$ & $4.48688 \mathrm{e}+01$ & $4.21468 \mathrm{e}-08$ & $1.72009 \mathrm{e}+01$ & $3.21128 \mathrm{e}+01$ \\
\hline std & $1.37309 \mathrm{e}-15$ & $0.00000 \mathrm{e}+00$ & $2.12703 \mathrm{e}-01$ & $4.01418 \mathrm{e}+01$ & $8.25906 \mathrm{e}-09$ & $6.38805 \mathrm{e}-01$ & $4.49296 \mathrm{e}+00$ \\
\hline SR & 100 & 100 & 0 & 32 & 0 & 100 & 100 \\
\hline vio & $0.00000 \mathrm{e}+00$ & $0.00000 \mathrm{e}+00$ & $5.05000 \mathrm{e}+01$ & $1.18429 \mathrm{e}+01$ & $7.29695 \mathrm{e}+04$ & $0.00000 \mathrm{e}+00$ & $0.00000 \mathrm{e}+00$ \\
\hline problem & $\mathrm{C} 22$ & $\mathrm{C} 23$ & $\mathrm{C} 24$ & $\mathrm{C} 25$ & $\mathrm{C} 26$ & C27 & $\mathrm{C} 28$ \\
\hline best & $7.82296 \mathrm{e}+01$ & $7.84209 \mathrm{e}-01$ & $2.35612 \mathrm{e}+00$ & $1.57066 \mathrm{e}+00$ & $1.00167 \mathrm{e}+00$ & $2.84751 \mathrm{e}+02$ & $2.38846 \mathrm{e}+01$ \\
\hline median & $2.12380 \mathrm{e}+04$ & $7.84225 \mathrm{e}-01$ & $2.35612 \mathrm{e}+00$ & $1.57066 \mathrm{e}+00$ & $1.04543 \mathrm{e}+00$ & $2.84829 \mathrm{e}+02$ & $5.38774 \mathrm{e}+01$ \\
\hline $\mathrm{c}$ & 100 & $\begin{array}{lll}0 & 0 & 0\end{array}$ & $\begin{array}{lll}0 & 0 & 0\end{array}$ & $\begin{array}{lll}0 & 0 & 0\end{array}$ & 100 & $\begin{array}{lll}0 & 0 & 0\end{array}$ & 100 \\
\hline $\bar{v}$ & $1.54193 \mathrm{e}+01$ & $0.00000 \mathrm{e}+00$ & $0.00000 \mathrm{e}+00$ & $0.00000 \mathrm{e}+00$ & $5.05000 \mathrm{e}+01$ & $0.00000 \mathrm{e}+00$ & $7.30594 \mathrm{e}+04$ \\
\hline Mean & $3.23001 \mathrm{e}+04$ & $7.84396 \mathrm{e}-01$ & $2.35612 \mathrm{e}+00$ & $5.27774 \mathrm{e}+00$ & $1.05214 \mathrm{e}+00$ & $2.99755 \mathrm{e}+02$ & $5.15351 \mathrm{e}+01$ \\
\hline Worst & $8.20487 \mathrm{e}+02$ & $7.87071 \mathrm{e}-01$ & $2.35612 \mathrm{e}+00$ & $3.29866 \mathrm{e}+01$ & $1.09745 \mathrm{e}+00$ & $3.18890 \mathrm{e}+02$ & $9.74830 \mathrm{e}+01$ \\
\hline std & $8.81380 \mathrm{e}+04$ & $5.65603 \mathrm{e}-04$ & $3.39107 \mathrm{e}-09$ & $6.63285 \mathrm{e}+00$ & $2.54681 \mathrm{e}-02$ & $1.68980 \mathrm{e}+01$ & $1.51348 \mathrm{e}+01$ \\
\hline SR & 48 & 100 & 100 & 100 & 0 & 100 & 0 \\
\hline$\overline{v i o}$ & $3.65205 \mathrm{e}+01$ & $0.00000 \mathrm{e}+00$ & $0.00000 \mathrm{e}+00$ & $0.00000 \mathrm{e}+00$ & $5.05000 \mathrm{e}+01$ & $0.00000 \mathrm{e}+00$ & $7.30575 \mathrm{e}+04$ \\
\hline
\end{tabular}

